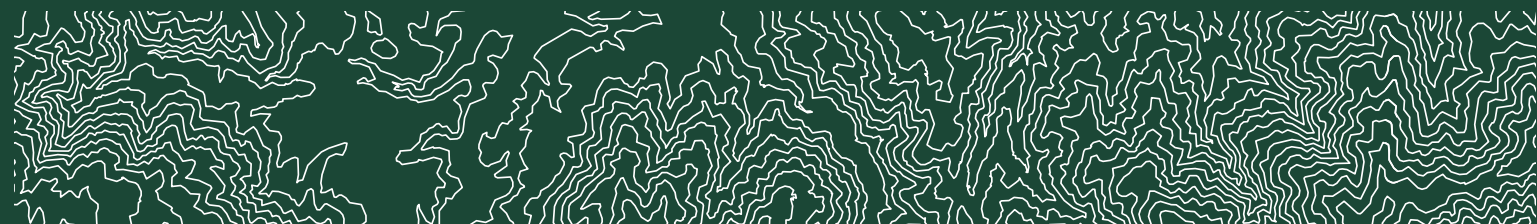

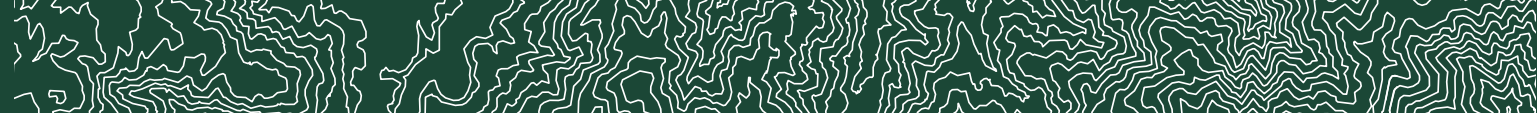
(2)

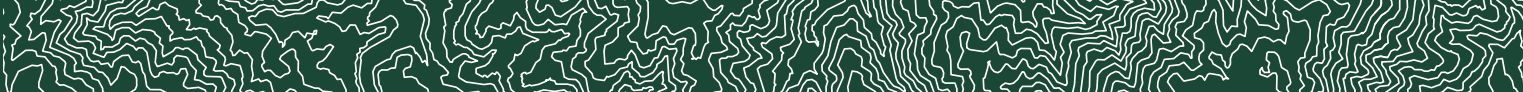

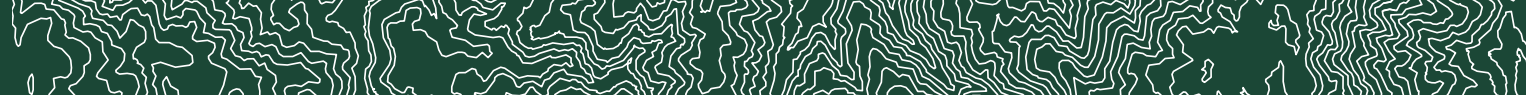

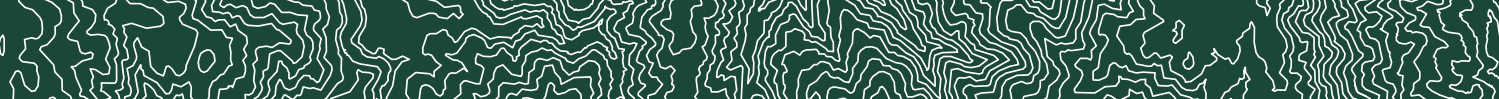
W 3.53 ( )

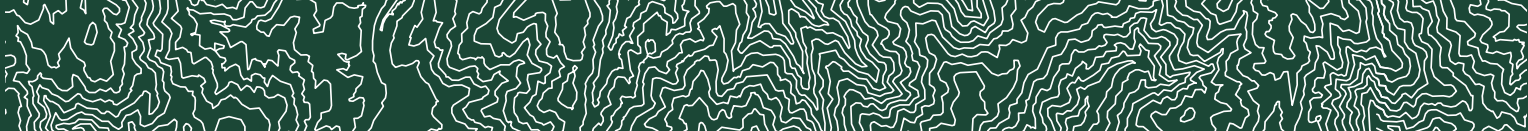
(1)

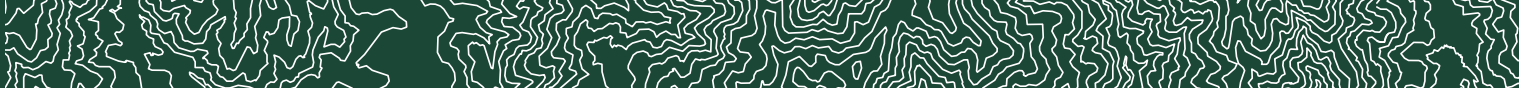

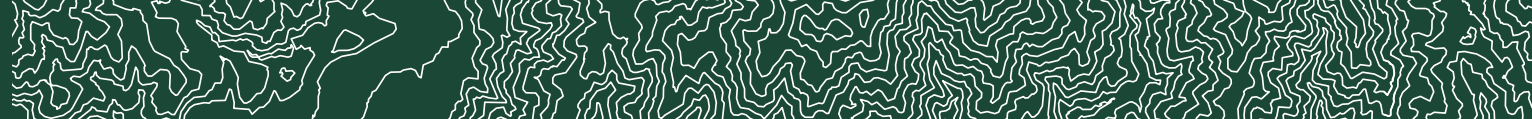
(1) (1) 


\section{Head for the Hills}

William Briscoe

A 120-point thesis submitted to the Victoria University of Wellington

in partial fulfillment of the

requirements for the degree of

Master of Architecture (Professional)

Victoria University of Wellington

School of Architecture 


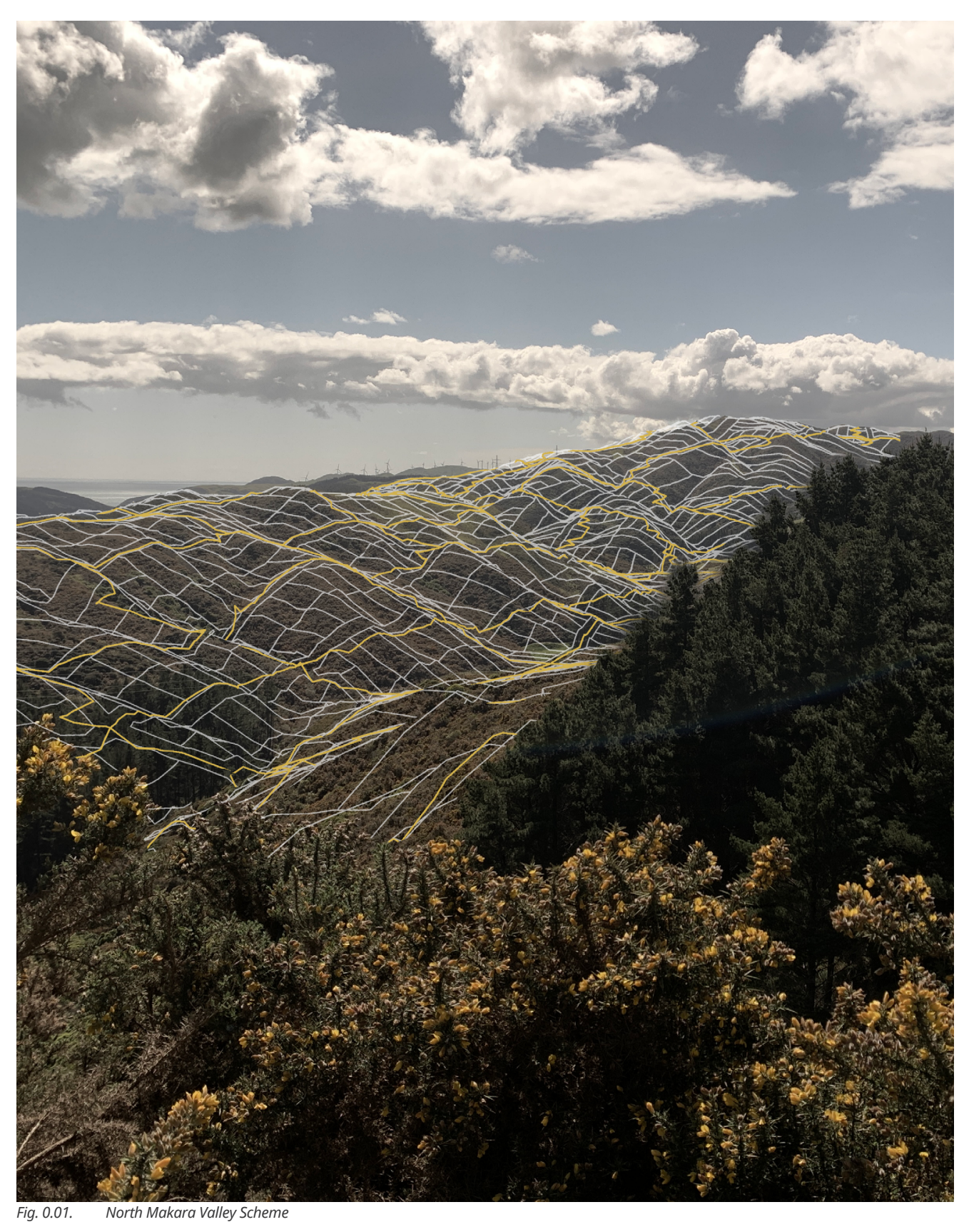

\section{Abstract}

In the context of urban design, generative design has been examined as a tool for expansion or optimisation of existing urban networks. This optimisation uses information such as geometry of the existing urban fabric and available space for expansion. However, very little research exists into designing around terrain factors, instead usually opting to consider difficult terrain as simply a boundary for network expansion

This research seeks to answer the question 'How can generative design improve the way urban networks are designed in complex terrain?' It does this by creating a tool that can interpret any terrain information, and with simple designer input, can create conceptual urban schemes in complex terrain.

The tool is developed using visual programming the Rhinoceros3D modelling software. Its development and proof-of-concept scheme are executed in Wellington, New Zealand. The city is one uniquely situated between harbour and steep hills, leading to several typologies of hillside urban schemes to use as precedent and comparison with the tool's outputs. The Wellington City Council Urban Growth Plan anticipates an increase of 80,000 people in the next 30 years, and the city requires additional areas to house the growing population.

Through a discussion of urban theory and existing generative design exemplars, the thesis settles on an urban grid-based logic for the tool. The thesis then records the process of designing the tool, using a Wellington site as a base for development.

Evaluation of the tool is undertaken using space syntax theory as a key framework, as well as qualitative comparisons with existing hill suburbs in Wellington. 


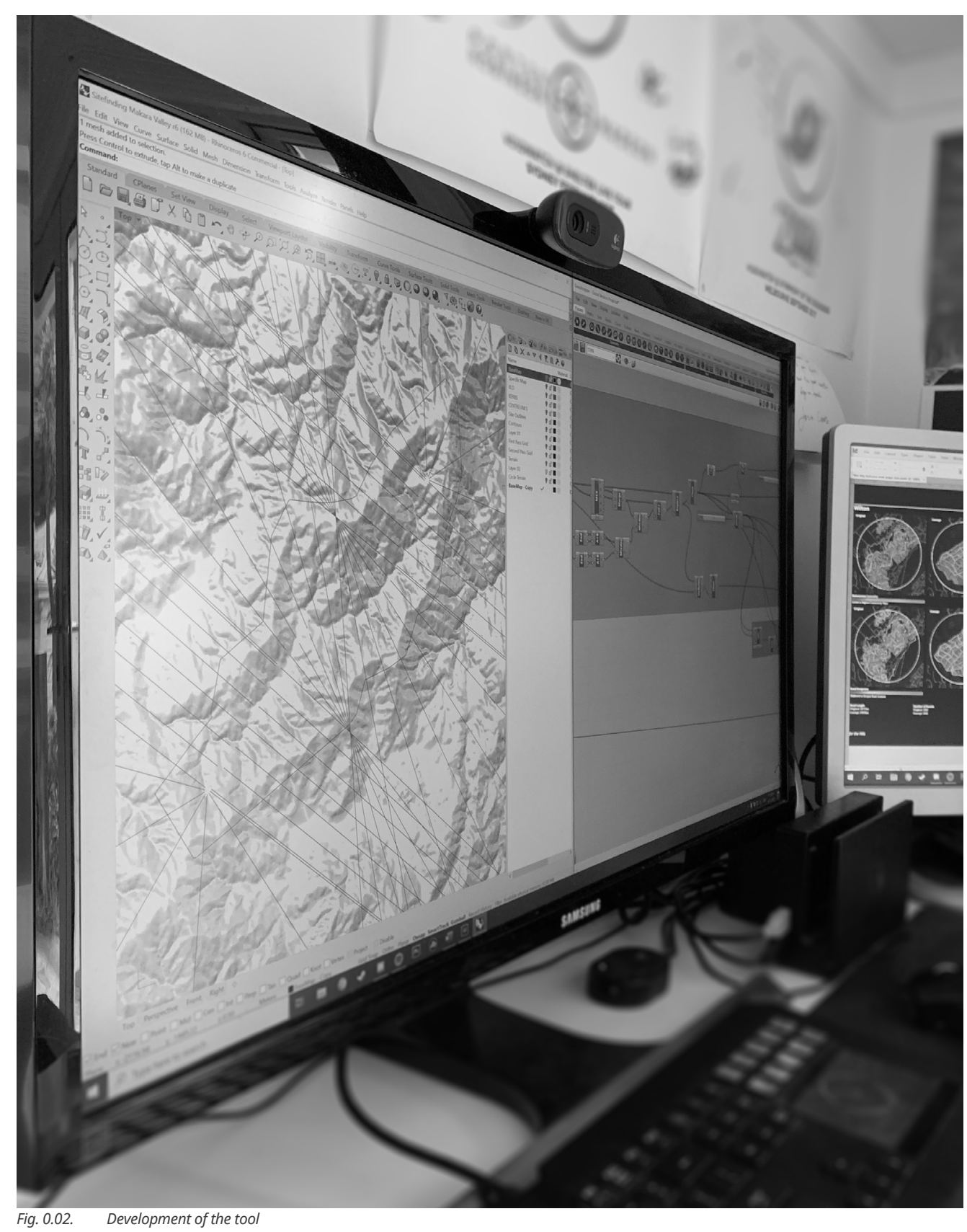

To Fabricio Chicca, my supervisor: your clarity of vision and industry experience were invaluable to this thesis. Thank you for your guidance throughout this year.

To my family, and especially my parents: thank you for your love, insight, willingness to get into the details, and frequent comfort food escapes. Not to mention all the LEGO!

Grace and Alice, I could not ask for better flatmates or better friends. Here's to me finally joining you in the real world.

Marina, thank you for keeping me on the straight and narrow. Architecture is a truly worldwide language and I'm so glad it brought us together. 


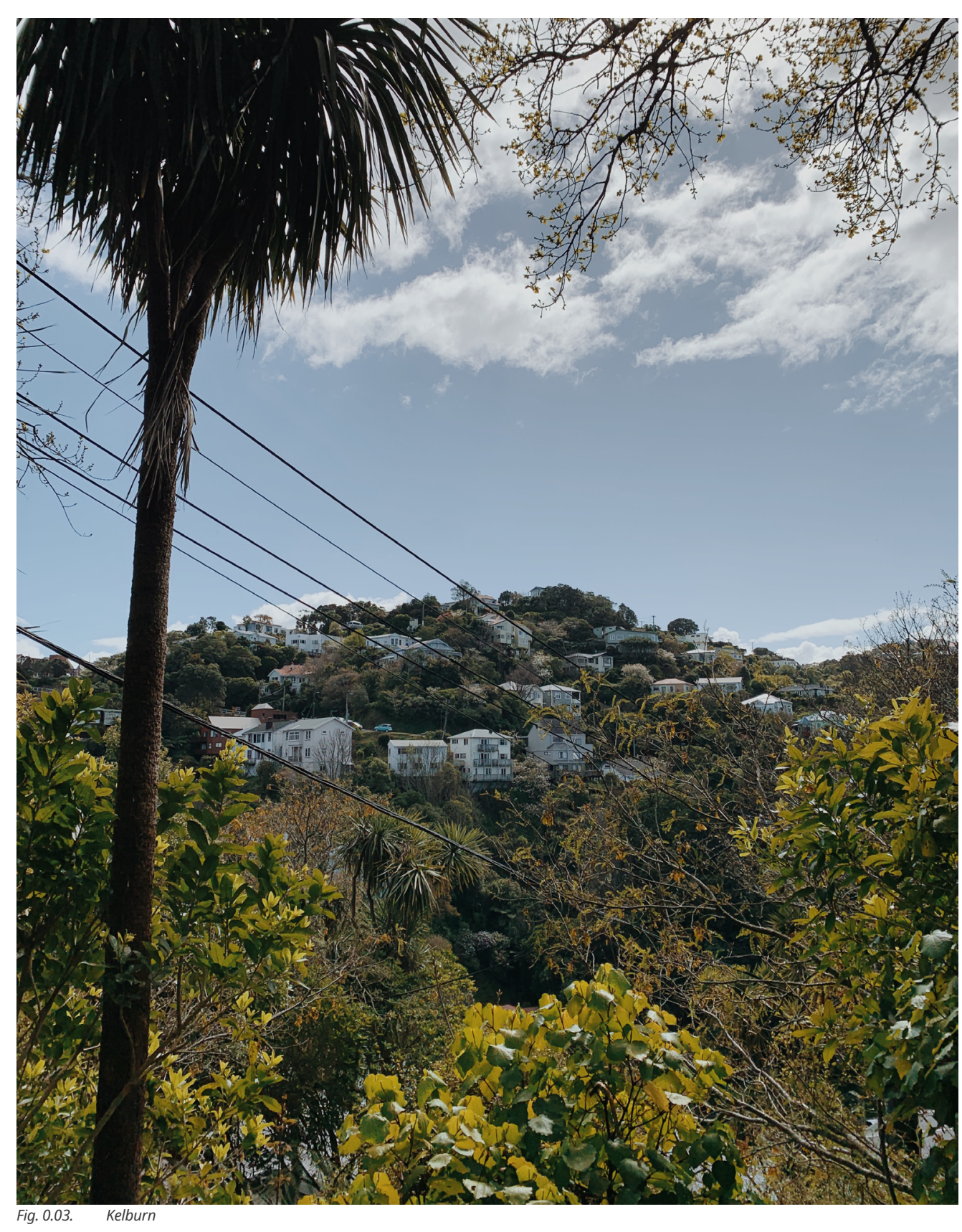

\section{Contents}

1. Introduction 1

Methodology 3

Scope

2. Background 9

3. Literature Review 16

4. Urban Logics vs Landscape Logics

Wellington Photo Journal $\quad 5$

Site

5. Prototypes

6. Proof of Concept: North Makara Valley 91

7. Evaluation 111

Rapid Concepts $\quad 116$

Limitations 126

8. Next Steps

9. Conclusion \& Critical Reflection

Bibliography 138

List of Figures $\quad 143$

Appendix $\quad 146$ 


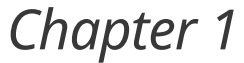 \\ Introduction}

As technology improves, designers' ability to harness growing computational power to solve difficult design challenges increases in turn. This manifests itself in different ways across the broad spectrum of design, but of particular interest is the emergent field of generative design. "Generative design combines parametric design and artificial intelligence together with the restrictions and data included by the designer", quickly creating iterative solutions to a design problem that can be moderated by the designer guiding the process (Souza 2020).

In the context of urban design, generative design has been examined as a tool for expansion or optimisation of existing urban networks (Koenig 2013). This optimisation uses information such as geometry of the existing urban fabric and available space for expansion. However, very little research exists into designing around terrain factors, instead usually opting to consider difficult terrain as a boundary for network expansion. This gap in the body of knowledge suggests a great deal of possibility. In order to make the most of available land, difficult terrain needs to be embraced as a possible space for urban environments.

New Zealand's capital city, Wellington, has been chosen as the focal point for this research due to the city's extreme geography. Built on a narrow strip of land between harbour and steep hillsides, Wellington has had no choice but to expand into difficult terrain and will likely need to expand further in the future. Population growth in the city will increase demand for housing, with Wellington anticipated to grow by 50,000 to 80,000 people over the next 30 years (Wellington City Council 2020). The need to pursue alternative sites is exacerbated by sea level rise due to climate change. New Zealand is predicted to suffer $5-10 \%$ greater rises than the global average (Ackerley 2013) and as such needs to begin preparing for where the residents of 43,680 homes (Bell 2015) will relocate to after their land becomes unviable.

The key question this thesis poses is 'how can generative design improve the way urban networks are designed in complex terrain?' Head for the Hills details the process of designing the generative urban design tool that addresses this question. The output of the thesis is a tool which functions as a computational urban design prototyping, or CUDP, tool (Miao et al. 2018). It focuses on how to bring rational order to complex terrain, bringing it in line with urban logics that take place on flat land. Despite using a New Zealand context for tool 


$$
\begin{array}{|l|}
\hline 1 . \\
\hline 2 . \\
\hline 3 . \\
\hline 4 . \\
\hline 5 . \\
\hline 6 . \\
\hline
\end{array}
$$

\begin{tabular}{|l|}
\hline 1. Terrain Modelling \\
\hline 2. Graphical Analysis \\
\hline 3. Urban Logic for Site \\
\hline 4. Road Placement \\
\hline 5. Building Parcel Placement \\
\hline
\end{tabular}

6. Further Detailing and Infrastructure

development, this research sets out to be adaptable and applicable to extreme terrain anywhere in the world.

The tooling process follows thesis Testing Terrain (Belesky 2018), which comprehensively discusses the epistemological implications of undertaking research through design by creating tools. The process of tooling is situated between the trinity of Research For Design, Research About Design and Research Through Design. Different parts of the process fit in better with different types of research, but the core part of the process is grounded in a research for design methodology. In the thesis' original landscape context, it aims "to help enable a specific type of design activity, rather than define normative theories of landscape architectural design...” (Ibid).

The environment selected for the development of the tool is Grasshopper, a visual programming language extension for the Rhinoceros 3D Modelling software. It allows for parametric control and development of geometry within Rhino in order to achieve complex design outcomes that can also be easily adjusted. Despite being executed within Grasshopper, the workflow embodied by the tool could be extended to other parametric environments or even a (time-consuming) manual process.

\section{Methodology}

The focus of the thesis is the design of the tool and the logic behind how it places roads and land parcels on steep sites. Due to the occasionally fickle nature of technology the work is structured in a way that would allow adaptation of the original research plan if a roadblock is encountered. Put simply, the methodology is structured around establishing whether something is possible, building it, then entering the appropriate parameters as determined by specific research in order to make the tool fit for purpose.

The thesis will follow a methodology as laid out in the diagram above.
The Grasshopper component of the tool development has been split into several steps that represent a workflow a designer might use to help to design a single urban plan. The parametric nature of Grasshopper means that with some adjustments the steps used to develop one urban scheme may be applicable to many sites.

Step 1 is terrain modelling. To ensure functionality across different topologies, the input for the remainder of the tool must be consistent in how it is modelled. Factors such as whether the topologies are digitized as meshes or surfaces, or the resolution of the information, affect how the tool will interpret the information. 
Step 2 is to have the tool analyse certain attributes of the topography. If the end product of the tool is to take into account factors such as solar exposure and site gradient, this information needs to be extracted from the 3D model. Wellington is a good example of a place where existing developments around hilly suburbs fail to account for sun exposure, one of a few disparate factors that account for the damp housing crisis in the city. One such example is Aro Valley, where $40 \%$ of houses are damp some or all of the time (Mckenzie 2020). This step's output is helpful for the person proceeding through the tool's workflow to visually understand certain aspects of the site, establishing whether the site is appropriate before continuing.

Step 3 will attempt to bring an order to the site that allows the application of conventiona urban design knowledge. Translating the linear nature of an urban grid system to the free-form of hilly terrain allows nodal points from which to structure the remainder of the tool's outputs. The relevant urban knowledge will be based on discussions within Public Places, Urban Spaces (Carmona 2003) and presented within the literature review chapter of this thesis.

Steps 4 and 5 are the difficult part of the development. Using gradient information, the tool will generate the most effective paths for roads for the urban grid, and then populate the roads with building parcels.

Step 6 is development time allowed in advance for extra layers of information that may be beneficial to add to the tool, as decided by evaluations later in the research process.

Once the tool is functional, the variables in the tool will be changed to reflect information collected through detailed research. Examples would be the maximum gradients for roads or the steepest possible site that could support a building. A specific site will be chosen in Wellington to provide an example of the tool's output, to be used to for demonstration and evaluation of the tool.

Throughout the process there will be constant referral to previous steps and evaluation of whether the tool is achieving a functional output. This will enable any additional steps or changes in development direction to occur over the course of the research.

\section{Scope}

As a relatively underexplored area within its wider field, this thesis represents a first step into terrain responsive generative urban design. Its primary concern is finding ways to consistently work on different terrain types to produce a viable outcome for a concept scheme. With this in mind, the tool will not proceed further than producing concepts, focusing on exactness instead of complexity. It is being produced as part of a single author Master's thesis and lacks the inter-university or industry support often seen in other papers in the field, and does not have access to coding resources to produce new extensions within text-based programming languages to expand Grasshopper's functionality. The tool relies on third-party open source plugins, and as such is not fit to be made into a commercial produc at this stage.

The tool itself has technical limitations, which will be discussed in Chapter 7. In term of design limitations, the tool will not be addressing aspects outside the scheme. While ultimately important to the success of any urban development, the external infrastructure that links it to the existing city network an improves capacity for transit to the city centre would need to be designed independently. While being able to report statistics about generated schemes, the tool will not be able to consider financial information during the generation process. Any financial constraints will have to be applied to the overall schem once completed, providing another metric by which to evaluate and modify the final scheme.

Living in extreme terrain is a research are which has the potential for great innovation, challenging the way people inhabit or circulate through space. To avoid diluting the planning and simulation-oriented focus of the research the thesis has opted to design for our curren level of urban circulation. Future advances in circulation in difficult terrain, like the introduction of alternative modes of public transport better suited to steep slopes such as funiculars or gondolas, could serve to furthe enhance the possibilities of the tool and its outputs.

\section{Thesis Structure}

\section{Chapter 2 - Background}

This chapter will provide a more detailed explanation of what Grasshopper is and how it works in the context of making tools for designers. This includes a discussion of how best to present the Grasshopper process and workflow in the thesis, and a list of third-party open source plugins that enable the tool to function. It also discusses why Wellington has been selected for development and application of this tool.

\section{Chapter 3 - Literature Review}

In this chapter, several key literature examples are identified and discussed. They vary in content across the full range of information applicable to this thesis:

- What urban knowledge to instil within the tool

- How tooling works in the context of a research thesis

- Whom the tool is being made for and whom it will be useful for

- Existing research on generative design concerning terrain

- Existing research on generative design in the Grasshopper environment

Each section comprises either a single text (with supporting research) or the relevant work of a particular author or group.

Chapter 4 - Urban Logics and Landscape Logics This chapter analyses the existing steep terrain typologies in Wellington. It does this initially 
through a series of graphic analyses which showcase the solar exposure and steepness of Wellington building footprints, and the steepness and connectivity of Wellington road network. The chapter then goes on to explore certain steep terrain typologies with a photo journal of select hill suburbs in the city, with conclusions drawn from the are explored about what logic to instil in the tool. It concludes by selecting a site that is most suited for the development of the tool.

\section{Chapter 5 - Prototypes}

This chapter presents and briefly discusse various small scripts that were developed as modular components of the overall tool. Mos of these prototypes went on to be incorporated in the tool, though some were experiments that were replaced or refined later in the process.

\section{Chapter 6 - Proof of Concept - North Makara}

While the previous chapter presented how elements of the tool work, this chapter details how to use them. Using the chosen site, this chapter goes through each step in the process of generating a scheme, starting from the map file inputs and ending with a complete scheme including road network and land parcels.

\section{Chapter 7 - Evaluation}

This chapter discusses the viability of the scheme generated in the previous chapter, as well as quick examinations of schemes developed for the suburbs covered in Chapter 4's photo journal. Aside from evaluating the quality of the schemes, it also evaluates the success of the tool and discusses its limitations.

\section{Chapter 8 - Next Step}

The next steps the tool could take are divided in two: ways to improve the tool's existing functionality, and ways to extend or utilise the information the tool produces. This chapter discusses these two possible paths based on the evaluation from the chapter before, and also suggests parallel research that might be valuable in future.

\section{Chapter 9 - Conclusion \& Critical Evaluation} This chapter summarises the thesis' findings, discussing its main successes and how the results of this research may eventually lead to real world urban schemes.

\section{Appendix}

The appendix contains screenshot-based and annotated documentation of the Grasshopper scripts for those familiar with the program. 


\section{onstar \\ Background}

This thesis seeks to build a methodology for working in extreme terrain anywhere in the world, yet in order to develop this methodology sufficiently there needs to be a base for development that can expand outwards. This chapter will explain the suitability of two elements of the research selected for the development of the tool: the methodology, which is being built as a tool in visual coding language Grasshopper, and the location, which aspires to be adaptable but initially is set in Wellington, New Zealand.

\section{Grasshopper}

Grasshopper is a visual programming languag extension for the Rhinoceros 3D Modellin software. It allows for parametric control and development of geometry within Rhino in order to achieve complex design outcomes that can also be easily adjusted.

Grasshopper was selected for the thesis as it is frequently used in cutting edge architectural research and has a host of plugins that enable it to interface with any facet of architectura design. It was described as "arguably the most popular computational design tool" in 2016 (Kilkelly 2016), and its usage has grown since then after moving from an extension to full-fledged function of Rhinoceros 3D 6 in 2018. In a single definition (the name for a GH file which is a collection of instructions), Grasshopper could interpret Geographic Information System (GIS) data, transform this data into a model of a landform, establish its position in the world and calculate its yearly solar exposure.

Visual programming languages (VPL) provide a more easily understandable way of visualising the steps involved in a program by operating in a graphical form instead of text. Grasshopper is classified as a Data Flow VPL, in that each component has inputs and outputs that linearly pass information along the definition (craft ai 2015). This makes the software highly suited to being used to make tools.

Data Flow VPLs are considered the most professional of the types of VPLs due to individual components being made in a conventional programming language and requiring a strong understanding of data structures being passed through the definition (Ibid). The tool is being designed in a way that streamlines its usage, but to fully troubleshoot and maximise the tools functionality the user will likely need some familiarity with the Grasshopper and Rhino environment. Most adjustable features of the tool will be presented 
as 'number sliders', so that the recommended range of inputs can easily be adhered to and nothing that should not be adjusted is touched by accident.

The logic behind my usage of Grasshopper follows that of Testing Terrain: Exploring the Computational Design of Natural System in Landscape Architecture (Belesky 2018) which explores a similar methodology to this research. He notes that "while the operative logic of each [GH Definition] is coupled to th Rhinoceros/Grasshopper environment, it is not inextricably tied to it” (Ibid). The workflow used in Grasshopper to perform one action may differ in another parametric software but the logic behind any particular step is transferrable to other software. To draw on Belesky's example, the process of creatin a circle in the Rhinoceros/Grasshoppe environment differs from the Revit/Dynamo environment:

Rhino.Geometry.Circle(new Rhino.
Geometry.Point3d(0, 0, 0), 1250)

As opposed to:

Arc.Create(XYZ.Zero, 1250, 0.0, 2.0 * Math. PI, XYZ.BasisX, XYZ.BasisY)

'The underpinning logic is roughly equivalent in each case - create a circle - even though each environment requires a different programmatic expression of that underpinning intent' (Ibid). Head for the Hills proposes not just a specific toolset created within the Grasshopper environment, but a workflow that could be carried out in another parametric environment or indeed a process that could be followed by conventional means, albeit with great difficulty.

How Belesky's work influenced this thesis will be expanded upon in the literature review section.

In this tool, users will generally interact with the Grasshopper script by modifying 'number sliders' to define variables. These will be referred to frequently in discussions of how the script works and how users can modify outputs. There will also be a discussion of Grasshopper data trees and how they separate the tool's information later in the thesis.

This thesis utilises the following plugins made by third parties, often as part of their own architectural or computer science research.

Ladybug (Sadeghipour Roudsari 2013)

Ladybug parses .epw weather files and allows for simulations using Grasshopper geometry to obtain preliminary site solar information.

\section{Anemone (Zwierzycki 2013)}

Anemone is a looping tool that allows for repeated steps within the Grasshopper environment. This can create additive transformations or simple agent based simulations.
Galapagos (Rutten 2010)

Galapagos is an evolutionary solving algorithm. By specifying a selection of variable number sliders (acting here as genes) and definin a 'fitness function' or measurable goal, the solver will be generate permutations using evolutionary logic to work towards an optima solution to the fitness function. The simulatio runs over time and constantly improves its result by 'breeding' successful genes together to work towards the solution.

Heteroptera (Bahrami 2014)

Heteroptera is a toolbox with a variety of features across five categories: Animation, Mathematics, Text, Uncertainty, and Networks. In this thesis only the network component is used to calculate the topology of the road network.

Weaverbird (Piacentino 2009)

Weaverbird is a mesh analysis and transformation tool that can refine, add complexity, or analyse meshes. In this thesis it is used for mesh analysis purposes.

DeCodingSpaces (Bielik et al. 2019)

DeCodingSpaces is a generative urban design toolkit. It will be discussed in depth in the literature review chapter of the thesis.

GHSHP (Hiteca 2017)

GHSHP is a simple component that allows Geographic Information System (GIS) shp files to be read in the Grasshopper environment. This allows a bridge from commonly available map data to mesh maps in Grasshopper.

Pufferfish (Pryor 2018)

Pufferfish is a large toolkit with components focused on complex geometry modifications. In this thesis it is being used for its convenient data handling components to reduce the bulk of the tool.

\section{Treesloth (Stasiuk 2015)}

Treesloth has components that enable more complex editing of data tree structures. This enables the tool to maintain a branched data structure throughout the workflow that can allow easy separation of key aspects of the output, in turn enabling specific sections to be edited or analysed. Without it, the data structure would be flat and retrieving specific sections of the output would be more difficult. The data structure of the tool will be elaborated upon later in the thesis.

Human (Heumann 2018)

Human has components specifically concerned with improving visualisation of the Grasshopper canvas and its output. In this research it is being used to highlight certain geometry within the tool for the user's benefit by allowing custom lineweight control within the Rhino viewport. This feature has also been used in preparing many of the graphics for this thesis.

Human does have capabilities of unifying controllable variables from the Grasshopper environment into one single control panel for ease of use. As this research is as much about 

decision has been made not to use this functionality. It is better for a user to follow the steps taken within the tool and adjust the variables in place for the benefit of future expansion of the research

\section{Grasshopper}

\section{Documentation}

While Grasshopper is a visual programming language and therefore visual by nature, understanding its data flow in a raw form is incredibly difficult even for seasoned Grasshopper users.
A City is not a Tree (Richards 2017) contains a discussion of how to best present Grasshopper definitions within a design thesis, which has been appended to reflect considerations more relevant to this thesis.

In the context of A City is not a Tree, Richards concluded methods 3 and 1 together were the most relevant way of presenting his definitions. The thesis revolved around generating generic city blocks and populating them with tall towers. Due to a lack of real world site and a randomly generated, seed-based output with many outputs displayed throughout the document, this choice is logical.
This content is unavailable. Please consult the figure list for further details.
With Head for the Hills, the slight difference in subject matter means that the best method of presentation may be different. While the tool is being designed to be totally adaptable, it is being developed with a site in mind to work as a proof of concept of the tool's functionality. As such, the best method will likely be a combination of 3, 4 and 1. Displaying the tool's output on the same site over the course of the workflow allows readers to clearly see the step by step process being undertaken without the need for a separate diagram. Textual and diagrammatic descriptions of the process assist readers in understanding the logic used between steps.

\section{Growth in Wellington and New Zealand}

While this thesis' applications are universal, Wellington has been chosen as a focus and source of a potential site. The city's unique position between steep hills and a deep harbour has resulted in necessary development of complex sites, but narrow roads, convoluted pathfinding and current congestion issues suggest there may be ways to improve the urban design of the parts of the city on stee gradients. "[Wellington's] street layout was largely derived from English town planning processes. Often it did not take the steepness of the land into account" (Wellington City Council 2011). Whether initial urban planners sought to bring order to the landscape with rigid grids, as in Mt Victoria, or to adapt to the landscape as best as possible, as in Kelburn, neither system presented a perfect solution to living in Wellington's landscape. The rigid grid can have incredibly steep roads where the landscape is not regular in slope. Roads that follow the landscape, originally farm trails used by early settlers that have since been widened (Kebbell and Ombler 2018), lack the connectivity in their network which in turn results in bottlenecks in modern transportation. The tool seeks to propose urban networks that tackle both issues and allow a maximization of land use in the

In the Draft Spatial Plan, Wellington City Council estimates the city is expected to grow by 50,000 to 80,000 people over the next 30 years (Wellington City Council 2020). Despite Wellington's steep terrain and limited flat land, this tool is not the sole solution for the city at present. A 2019 consultant's report preceding the Draft Spatial Plan provided Wellington residents with a range of scenarios that they could record their position on. The two most popular scenarios were the ones that focused on development in existing suburban centres (66\% agreed or strongly agreed) or the central city (58\% agreed or strongly agreed). Scenarios that prioritised greenfield development in Ohariu Valley (24\% agreement) and elsewhere around Wellington (29\% agreement) received comparatively little support, indicating that 
Wellingtonians appreciate an increase in density is better for the city (Global Research 2019).

Even with this taken into account, aiming towards greenfield development makes the most sense for the design of the tool as it allows terrain deemed too challenging for usage to be explored, and removes limitations of existing buildings that may inhibit or overcomplicate development of the tool. An increase in density in the central city is unlikely to be sufficien to house so many new Wellingtonians, and so there will likely be a place for the tool and for new greenfield development in the future.

By limiting this development to sites that are difficult to use for other purposes, hopefully this tool will help to mitigate the usual harms of greenfield development. If the tool were to be expanded upon to be relevant to Wellington right now, it would benefit from the functionality of being able to explore smaller areas of unexploited land within the core of Wellington to improve the density of the city. With that said, New Zealand is unusual globally with a low population density of only 18 people per square kilometre (World Bank 2018). If New Zealand had the same density as, for example, the United Kingdom (at 294 people per square kilometre), it would sustain a population of 74 million instead of 4.9 million (World Bank 2019).

In the future, more land will need to be used for housing both in Wellington and throughout the country. This tool will enable difficult sites to host that housing, instead of flat land that is needed for agriculture, nature preservation or other functions impossible on hilly terrain. The density of urban plans produced by the tool is something that will be able to be defined, ensuring versatility in its outputs that can be suited to all development types.
This content is unavailable. Please

consult the figure list for further

$$
\text { details. }
$$

Fig. 202.

'Clambering up the sharp ridges and rambling down into the deep gullies of the foot of the northern island of New Zealand, there is an unusual city. A determined city, that has elbowed a space for itself when there was none, and bound itself on to the inhospitable hills with roads that twist down to a blue enclosing harbour.'

'At first, settlement was a game of follow the leader along the ridges. But soon, there were more players than rules. Now it looks like citizens compete to see who can perch a home in the most precarious position.'

Stills and quote from 'Toehold on a Harbour', Ryan 1966 


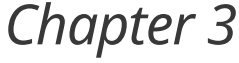 \\ Literature Review}

Now that the context for the development of the tool is set, the next step is to establish the procedure and logics that the tool will follow. In order to do this, a wide host of literature has been reviewed across the breadth of the spectrum of design: urban design theory, epistemological considerations, tool agency and user groups, and comparable generative design research. This literature comes together to guide both the logics and processes of the tool. Each section comprises either a single text (with supporting research) or the relevant work of a particular author or group.

\section{Urban Knowledge}

Public Places, Urban Spaces Matthew Carmona, Steve Tiesdell, Tim Heath, Oc Taner 2003

Urban design is a complex and multifaceted discipline that would be impossible at this level of technology to reduce to a simple set of instructions carried out by a computer The tool developed in this thesis needs to be able to negotiate a solution that fulfils critical urban design metrics that make sense within the context of difficult terrain. Public Places,
Urban Spaces (Carmona et al. 2003) contains a comprehensive discussion of all aspects of urban design and provides a strong repository of information from which to select the urban logics that steer the tool's development. There are two chapters within this book that are particularly relevant to this thesis, both within Part 2: The Dimensions of Urban Design. These chapters are The Morphological Dimension and The Functional Dimension.

The Morphological Dimension focuses on the configuration of urban form and space, and the spatial patterns of infrastructure that support it'. This tool seeks to bring a logic to urban design on complex sites that is comparable to the functionality of a successful urban network on flat sites. The key difference between the flat and complex sites is their morphological dimensions, as the typical layout and connectivity found in, for example, a regular urban grid, is impossible on a complex site due to gradient constraints.

Two keys ways in which development of urban space begins are either through the development along a main circulation route, such as the development on burgage plots as seen in English medieval towns (Conzen 1960),

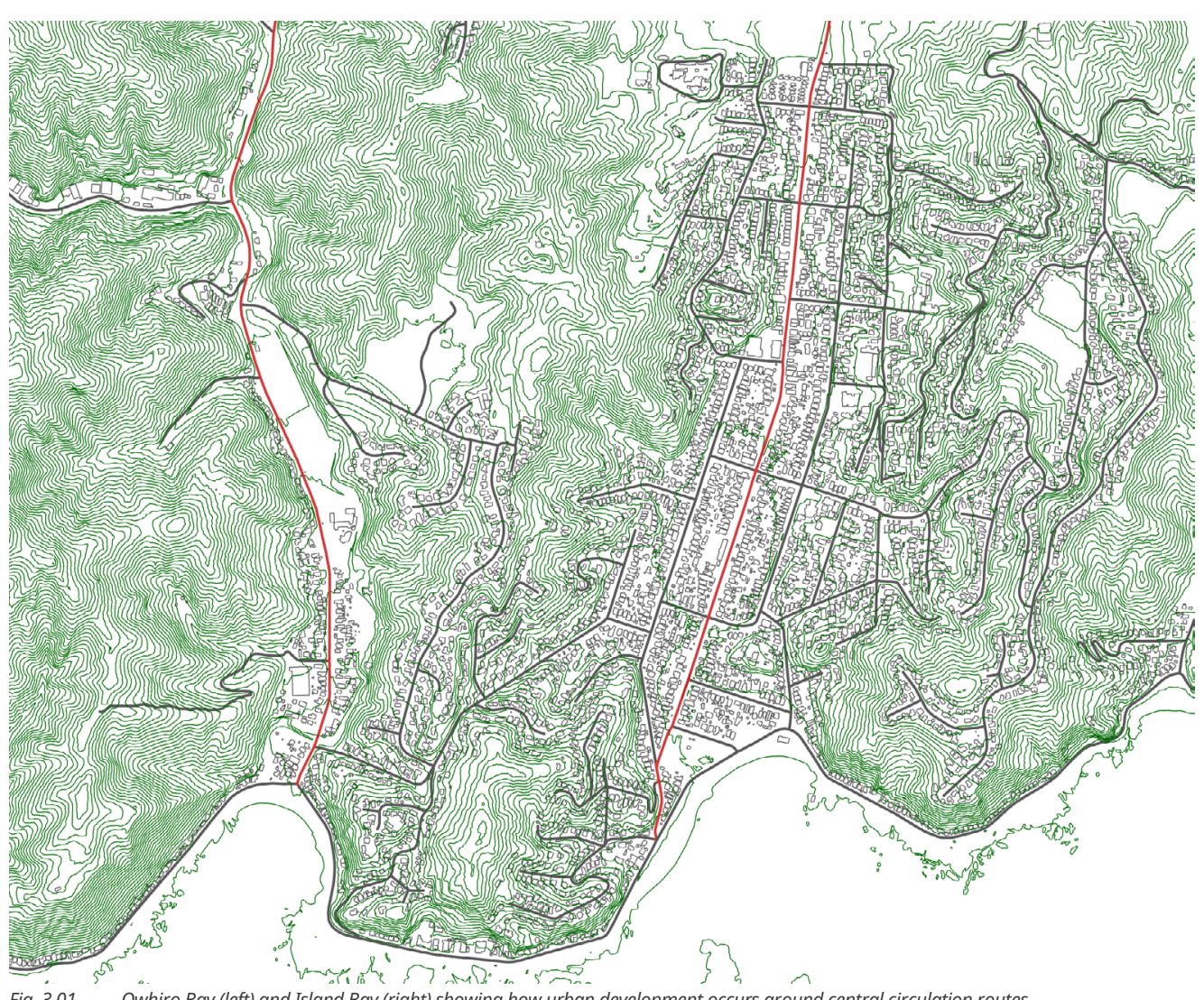

or in the case of places with no such existing routes, the urban grid. These two typologies are the most relevant to the tool as it is intended at this stage for new greenfield development.

While Wellington may not have literal burgage plots, both these typologies can be seen within the city. In the case of burgage plot style developments, a strong side by side comparison can be seen between Island Bay and neighbouring Owhiro Bay. Initial urban development occurs around key circulation routes, gradually forming blocks as perpendicular roads link together. Island Bay is a more mature suburb in this sense, whereas Owhiro Bay, constrained in a tighter valley, has less branching roads within its valley or on the neighbouring ridges. This style of development is suited to the ridges and valleys of extreme terrain but does result in a network with low connectivity, as it encourages central arterial roads that follow the landforms.

In terms of urban grid style developments, 
Mt Victoria, Hataitai and Mt Cook are good examples. While both typologies mature into blocks over time with organic growth occurring around the fringes, the best result will be achievable when planned out in its entirety and not left to natural growth. As such, planning for an entire urban grid system would produce a better result.

Carmona also identifies two distinct types of urban space:

- 'buildings embedded in urban blocks defining streets and squares... [with] interconnected, small-scale, finely meshed street grids'

- buildings as separate freestanding 'object-buildings' standing in an amorphous 'space' [within] large-scale road networks surrounding superblocks with discontinuous road layouts within'

The transition from traditional urban space (the former) to modernist urban space (the latter) took place in the second half of the twentieth century (Pope 1996, Bentley 1999). Modernist urban space tends to have been designed around vehicular circulation, and this is also true of some suburbs in Wellington. These locations are characterised by wide roads and ample car-parking space, though sometimes at the expense of adequate pedestrian space.

Carmona notes that "containing only movement space, roads divide and separate areas" (Carmona et al. 2003). This thesis would assert that this statement is not as true in
New Zealand as it may be in other parts of the world: Suburban roads in New Zealand are not the barriers they may be in other locations. Low population densities mean roads have relatively few cars travelling on them during the day, allowing a greater freedom of movement and more pedestrian-friendly environment. Whether this can continue in future when population density in Wellington increases remains to be seen. The experiential quality of Wellington suburban roads can be seen in the photo journal later in the thesis.

The next highly relevant chapter is The Functional Dimension, which discusses factors that affect the functionality of a successful urban scheme. The chapter examines urban functionality through two different perspectives: the visual-aesthetic tradition, which ‘drawing on the physical sciences... often [is] abstracted out and reduced to technical or aesthetic criteria, with function considered in terms of daylighting, over-shadowing, traffic flow, access and circulation, etc' (Ibid) and the social usage tradition, which examines 'how the design of the environment supported its use by people' (Ibid).

In terms of this thesis, the most relevant portion of this is the visual-aesthetic tradition and in particular how it studies movement. Within the process of developing a tool that relies on quantitative site information, it makes sense to stay within the realm of physical sciences.
Within the functional dimension of urban space, the tool is most concerned with movement. In this iteration of the tool, the outputted schemes will be at a concept stage, and thus unlikely to have enough detail to go beyond the most basic considerations of land use and infrastructure. However, a complete road network will enable speculative analyses of movement, particularly through Space Syntax. Space Syntax is an area of urban analysis that asserts that movement densities can be accurately predicted by analysing spatial configuration and the structure of the urban grid (Hillier and Hanson 1984).

The concern for movement is summarised with "If a space is poorly located within the local movement pattern, it matters little how well it is designed as it is unlikely to ever be well used... Thus, if a space is well located, then good design can enable it to realise its... untapped potential" (Carmona et al. 2003).

Movement in complex terrain is highly complex due to the constraints of both pedestrian and vehicular movement in a vertical direction. Building on hills actually provides an advantage to pedestrian movement over vehicular movement - able humans on stairs are able to move up steeper gradients than vehicles. Dedicated pedestrian spaces are beneficial for urban spaces and there is a 'symbiotic relationship between pedestrian movement and economic, social and cultural exchange and transactions' (Ibid).
In Wellington where town planners failed to anticipate the effect of gradient on the urban grid, the result was a network of 'paper roads', pedestrian roads that never became fully fledged roads and thus which only exist on paper (Cooke 2020). These accessways have become beneficial to the overall Wellington urban scheme by making the city more walkable, separating pedestrian and vehicular circulation, and making the most of pedestrians' greater ease of movement in steeper areas. This precedent could be followed by the user of the tool, informing their decisions as to whether certain circulation routes are roads or footpaths and allowing for good pedestrian connectivity within the urban scheme even over sites too steep for vehicular access.

Accessibility will remain a challenge for movement in steep terrain. Complex sites are by nature difficult for those with impaired movement, and stair networks or steep sidewalks on roads will cause further difficulty. Wellington's gradient limit for roads is meant to be $12.5 \%$ (though there are many exceptions in the city) in order to ensure streets are accessible for wheelchair users (Wellington City Council 2012).

Dealing with these issues may be outside the scope of the tool but instead up to the person evaluating the resultant scheme. In any proposal there may be areas of lesser gradient, which will then be more suitable for public or commercial areas, or to host houses aimed at people with impaired mobility. The tool should be able to visualise this information in order 
to enable designers to make these evaluative decisions.

On evaluation, the previously mentioned Space Syntax theory will serve as an important evaluative tool for any outputted urban schemes. Typically, Space Syntax deals with axial lines, "the longest and fewest set of lines of sight passing through all the convex (open) spaces in the area" (Carmona et al. 2003). The key logic behind this is that when pathfinding, pedestrians tend to favour routes with visual permeability, and are more likely to follow an axial line than turn at a junction. In difficult terrain, line of sight becomes a more complex equation. The terrain may provide a lesser or greater ability to see available routes at different positions. As a result, instead of reducing the scheme to axial lines, the road network will be analysed as is. The analysis that will be used is called Betweenness Centrality (Freeman 1977), which describes the frequency a certain path is used in the shortest route between any two nodes. This analysis provides a good visual representation of where the most important roads in the system are, which is particularly relevant for understanding where traffic and congestion will occur in the system, and which areas might benefit most from commercial or civic land uses.

\section{Tool Development as \\ Design}

Testing Terrain: Exploring the Computational Design of Natural

Systems in Landscape Architecture Philip Belesky 2018

In his $2018 \mathrm{PhD}$ thesis, Belesky comprehensively discusses the epistemological implications of undertaking research through design by creating tools. He situates the process of tooling between the trinity of Research For Design, Research About Design and Research Through Design. Different parts of the process fit better with different types of research, but the core part of his process is grounded in a research for design methodology. In his landscape context, he aims "to help enable a specific type of design activity, rather than define normative theories of landscape architectural design...” (Belesky 2018).

While the core methodology is research for design, that is not to say there is no design going on; Belesky alludes to other papers that establish tooling as a design process in itself. This tooling process could be considered “design for design" (Fischer 2008). Belesky seeks to "establish a close dialogue between the for and through modes of knowledge production that occur during design" (Belesky 2018).

Ranulph Glanville, a theorist frequently referenced in Belesky's writing, sought to differentiate between two approaches in computational design: that of design tools and design media. Design tools "[obey] our commands" and carry out the tasks they are made to do in the same way a person carrying out that task would, though usually in a faster and more efficient way. A design medium is when the computing tool is "participating and informing" and changing the output of the human designer by virtue of its method (Glanville 1992).

With the advent of generative design this split between tool and medium has been severely blurred because the tools have evolved from the "automated draftsman" (Glanville 1994) that CAD software was at its inception. Parametric design enables designers to create schemes that are no longer constrained by what humans can achieve with pencil and paper.

On this spectrum of tool to medium, Head for the Hills is positioned more on the side of the tool. It seeks to analyse and optimise, producing conceptual schemes that a person could conceivably design by hand but at a far faster rate. Tying analytical functions to the tool expands its usefulness as an estimator of financial and infrastructure statistics that operates faster than one individual designer.

As outlined above, that is not to say that the computer is not complicit in the design process. The finished script will 'participate and inform' the process in line with Glanville's description of a design medium. It could be argued that it is the landscape of the chosen site that is driving the design, but the same is true of any good site-responsive design. The tool's attempt to bring order to the complexity of a natural landscape requires it to have its own methods and logic. There is also a degree of the intent of the designer enshrined within the code that steers the output, a concept which will be covered in the next section.

\section{Who is the tool for?}

Stakeholder Views on Value and Urban Design

Matthew Carmona, Claudio De

Magalhães, Michael Edwards 2002

This tool is most appropriate for use at a concept stage of an urban scheme to ensure individual sites are well placed under several criteria: adherence to certain building codes, zone plans or climatic conditions. Simulation and optimisation tools strive for notions of perfection, though that ideal is impossible in the qualitative realm of architecture. This is exacerbated by that different stakeholders of an urban development have very different goals for a project, and as such very different ideas of what perfection could be.

Carmona et al. (2002) provides a good analysis of stakeholders in urban projects: their main motivations and their concerns over the actual quality of the design. 
This content is unavailable. Please consult the figure list for further

details.
This content is unavailable. Please

consult the figure list for further

details.

While the table was based on several sources, the main source drawn on by Carmona was the "most comprehensive stakeholder-based study undertaken in the UK" (Ibid) by the Royal Institute of Chartered Surveyors (RICS) and Department of the Environment (DoE) in 1996. This study was based on several office developments around England but can be applied easily to residential developments as

This clear difference in goals held by stakeholders in a project indicates a different possible 'perfect outcome' for each party. As such, being able to develop a tool that somehow caters to all potential users' needs is unlikely. Options will need to be examined. For example, the ability to select the density a which the tool places sites might make it more appealing to a developer (seeking high density and likely higher yield) and a typical NZ homeowner (probably seeking lower density) simultaneously. However, having the too generate geometry accounting for financi factors is currently outside the scope of this thesis. The tool might be able to output what these costs could be after the design workflow has completed, but it will not steer the design.

The three key stakeholders in the creation of a development are the developers, designers and council. From the table above, each has a central drive, but each of these three parties may somewhat desire what the other parties have as their main driver insofar as it improves their own in turn. For example: high building performance or strict adherence to codes even at concept phase can both help to increase the profitability the developer seeks. However, more often than not, developers seek the bare minimum in terms of quality in order to truly maximise their yield. As long as the council's rules are adhered to, they have no objections to this.

This is where the 'I-methodology' (Akrich 1995) comes into tool development. The I-methodology is where designers, often subconsciously, 'consider themselves as representative of the users'. This unconscious bias permeates the way in which the tool is understood, operated and the ends it 
seeks to achieve. The author's familiarity with Grasshopper could result in a tool too complex for the layman to properly wield, or the architectural and worldly perspective of a student could result in a tool suited only to designers in similar situations.

Whom, then, should the tool be for to make it as useful as possible? Instilling the author's idealism in the tool would generate schemes well suited to living but potentially not to building and selling. The final party involved is the end user, and in this development style the have little say on the end product. Protecting their interests is important and one of the initially stated goals of this tool. There are many pieces of research undertaken to challenge the status quo of development and which makes the case for investing heavily in sustainable material use and building performance (Saris 2017) that improve environmental impact and the lives of the end user. This tool is being developed on the shoulders of this research. It is for councils, developers and designers to utilise, but still seeks to protect the interests of the future residents.

\section{State of the Art: \\ Difficult Terrain}

This thesis is positioned adjacently to two pieces of research that start to consider the simulation of urban schemes on hills: Santa Marta Urban Grammar: Towards an understanding of the genesis of form and $A$ Grammar-Based Generative Urban Design Tool Considering Topographic Constraints. The key difference between this thesis and research that represents the state of the art is the framework used to generate geometry. While all under the umbrella of parametricism, the state-of-the-art research uses shape grammars, a specialized ruleset for creating geometry, to describe how an urban scheme might evolve.

As a concept, shape grammars began in Shape Grammars and the Generative Specification of Painting and Sculpture (Stiny and Gips 1972), but the groundwork for them as an architectural or spatial tool was laid out in Two Exercises in Formal Composition (Stiny 1976). In this article Stiny demonstrated how grammars can be used to describe an existing formal composition, transferring a designer's logic into a mathematical ruleset. He then demonstrated that if formal composition can be made into a language, new formal compositions can be devised using that language; that these rules can be used to generate similar compositions or projects infinitely.

Beirao and Duarte selected shape grammars as their chosen medium for the following reasons:
This content is unavailable. Please consult the figure list for further details.
- Analytical purposes where design rules and processes are understood

- Synthetic purposes where new design rules are generated

- Regulatory purposes where the limitations of new design alternatives are controlled - Prediction purposes where simulation algorithms are developed accordingly (Beirao and Duarte 2018)

The usefulness of shape grammars for advanced simulation or speculation was advanced by Unveiling the structure of the
Marrakesh Medina: a shape grammar and an interpreter of generating urban form (Duarte 2007) which developed a ruleset that described a complex and human-driven expansion process. The methodology had been previously applied to simpler 'languages', such as more modern urban schemes like the modern city grid, or classical, logical compositions like colonnades. By successfully finding a ruleset for a chaotic ancient urban scheme that had grown organically over time, the potential for this system to be applied to other unique situations has improved. 
Santa Marta Urban Grammar: Towards an understanding of the genesis of form

Debora Verniz and José Duarte 2017

Santa Marta Urban Grammar takes part of the logic from the Marrakesh Medina and applies it to another organically growing urban typology but adds the additional complexity of considering terrain. The paper focuses on the Santa Marta favela in Rio de Janeiro, Brazil. Using a digital model of the favela made from aerial photos, Verniz and Duarte identify the driving forces that determine how the urban plan grows:

- Topography, which 'works as a barrier to the growth of the formal city, which tends to occupy flat or slightly sloped surfaces. leaving [other] areas empty for occupation by the informal city'

- Urban context, 'as the placement of buildings is influenced by existing buildings'

- Functional Organization, 'as it constrain how buildings are shaped and sized'
Using these forces, shape grammar 'rules' were devised to generate buildings from limited initial geometry. This geometry began as the existing buildings on a site, the borders of an existing street, and the contour lines of the site. From this starting point, the shape grammar rules proliferate additional dwellings, with each new dwelling applying the ruleset to the updated environment around it to inform its shape.

According to the paper's conclusion, the 'key to the definition of the grammar was identifying the topological relations between buildings and pathways, and between adjacent buildings, as well as recognizing the influence that factors like topography, urban context, and functional organization have on site occupation'.

While Santa Marta Urban Grammar is highly relevant to this thesis, the shape grammar definition is still primarily concerned with the existing built fabric as opposed to the topography of the site.
This content is unavailable. Please consult the figure list for further details. 
A Grammar-Based Generative Urban Design Tool Considering Topographic Constraints

Mina Rahimian, José Beirao, José Duarte, Lisa Iulo 2019

The second paper presents a shape grammar at an urban block scale as opposed to that of individual dwellings. As such, it is more suited to focusing on topography's influence on the urban plan. The paper uses the Californian city of San Diego as its case study and is built as an extension for Beirao's CItymaker tool.

This paper identifies three different key typologies in San Diego's urban schem that exist depending on their underlying topography.

- Organic Grids: "are often created where the topography fluctuates considerably within the region limits and close-tonarrow ridges and alleys are observed. I organic grids, buildings are constructed on the ridges and alleys where minimum construction standards are met"

- Orthogonal Grids: "are normally planned and constructed where a massive area of the site is flat or has a limited steepnes. According to San Diego's GIS data, a flat area is where the slope is less than $15 \%$.
An orthogonal grid is made of a series of parallel streets which run at right angles to each other forming a grid"

- Mixed Grids: "are the combinations of the previous two typologies. Mixed grids emerge where the orthogonal grid meets the edge of a steep slope and gets distorted, organically, in consequence"

The research then develops a shape grammar tool primarily concerned with the latter two typologies. The tool locates the border of when the grade of a slope exceeds $15 \%$ to establish a boundary of the orthogonal grid, then uses the CItyMaker tool to populate this boundary with structured city blocks and buildings.

Once again the paper does not completely align with the aims of the thesis: while both strive to bring the order of a grid to difficult terrain, the San Diego shape grammar stops where the slope exceeds $15 \%$. This thesis aims to utilize the land that would otherwise be considered too steep to properly build on. The most useful aspect of the paper is its analysis of the three typologies in San Diego. Wellington has these typologies but they can be divided into further categories, as the next chapter will examine.
This content is unavailable. Please

consult the figure list for further

details.
These papers have provided a strong starting point for this thesis. Representing the state of the art for generative urban design responding to terrain, they highlight a gap in the body of knowledge: the need for a tool that can focus on and address complex terrain without additional anthropological inputs. Their usage of shape grammar highlights its power as a medium for generating form, but there is potential for research in this area using techniques other than shape grammars. By approaching the question of generative urban design from the direction of macro-scale urban layout rather than the micro-scale geometry focus that shape grammars require, new learnings can be found.

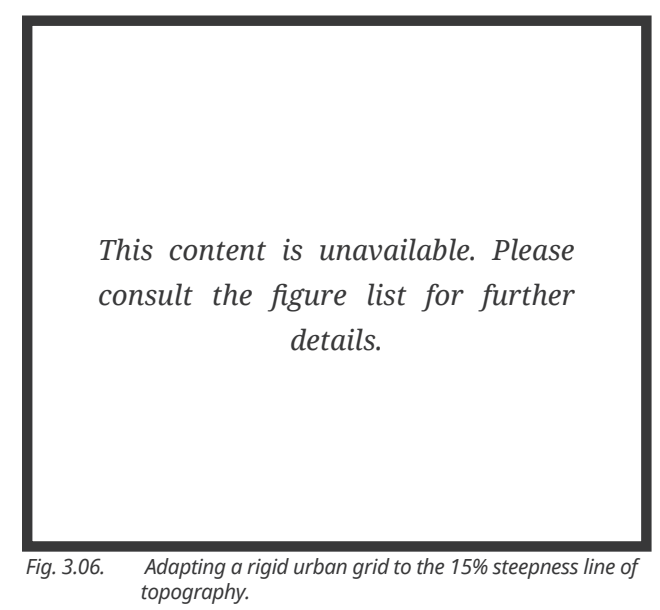




\section{State of the \\ Art: Generative \\ Design within \\ the Grasshopper \\ Environment}

There is research ongoing into generative urban design outside of the realm of shape grammar. Within Grasshopper, the state of the art components are developed by the Computational Planning Group as part of the plugin DeCodingSpaces Toolbox. This toolbox is a CUDP (Computational Urban Design Prototyping) tool, like the tool produced by this thesis. The following papers have been produced by members of the Computational Planning Group as proof-of-concept research demonstrating the functionality of certain aspects of the plugin

Graphical smalltalk with my optimization system for urban planning tasks

Reinhard Koenig, Lukas Treyer,

Gerhard Schmitt 2013

One of the plugin's functions is the generatio of street networks as defined by this paper. Streets are generated from 3 rules: the length of streets, the angle between streets at a node, and the number of streets possible on a node. This logic is applicable to every node in the system, and can also take into accoun existing streets, allowing a proposed solution to properly relate to existing infrastructure.
The application and tuning of these rules are controlled by a genetic solving algorithm, which sim steers the solution using evolutionary logics (Rutten 2010). Genetic solving algorithms require a 'fitness function' to work towards, which in this case is a measure of Betweenness Centrality or Choice, a space syntax term that describes the frequency a certain path is used in the shortest route between any two nodes (Freeman 1977). The solver will prioritise solutions that have higher net choice values, where the network is not extremely reliant on single paths for circulation as these paths would experience heavy congestion in real world examples.

This content is unavailable. Please consult the figure list for further details.
While a good workflow for Grasshopper and a system that works well on flat land, there is currently no provision within this plugin for adapting to terrain. The other issue with this plugin is that its mass-simulation based nature results in an incredibly long calculation time. As a parallel example of how to execute generative plans in Grasshopper, there will be a full discussion of the difference in final workflows between this research and my research in the conclusion.

Computational urban design

prototyping: Interactive planning

synthesis methods-a case study in

Cape Town

Yufan Miao, Reinhard Koenig

Katja Knecht, Kateryna Konieva,

Peter Buš, Mei-Chih Chang 2018

Another generative function of the plugin is its ability to divide blocks into parcels. This component works by recursively slicing the block as much as possible until a minimum width is met for each parcel. This slicing had to be executed in a way that would still allow every parcel to have a connection to a street.

This paper describes a case study in which both the street and parcel generation tools are used in a real example. The researchers looked at developing a new urban layout to replace an informal settlement in Cape Town, South Africa as part of the Empower Shack project. Beyond generating the urban plan, further steps were taken in the research such as developing a preference-based clustering algorithm that would allow residents to maintain existing social connections when they move from their informal dwellings into the new urban plan.

This content is unavailable. Please consult the figure list for further details.

Fig. 3.08. One proposal for the Cape Town project produced using

This paper provides excellent insight into how a computational urban design prototyping tool can be expanded upon to provide a comprehensive concept for any given site. While not regarding terrain, it is adjacent to this thesis in terms of its further application and additional internal steps. The parcel generation component is also something that could be utilized by my tool.

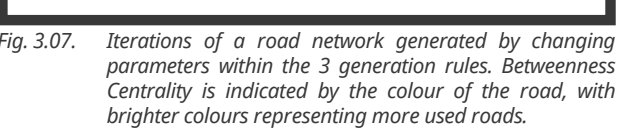




\section{Summary}

This chapter covered a broad section of research that relates to the thesis from different angles. Public Places, Urban Spaces discussed aspects of urban design that are able to be instilled within the tool and other aspects which should be left to later designers, allowing a clarity of focus for the tool's initial outputs. Testing Terrain discussed the epistemological aspect of making tools and how that sits within the design discipline. Stakeholder Views on Value and Urban Design looked at whom the tool might benefit. The works of Beirao, Duarte and the Computational Planning Group situated the tool in the realm of generative design and highlighted the need for a generative urban design that specifically addressed terrain in its logic

A key advantage of utilizing the Grasshopper environment for this tool is its compatibility with other generative urban design projects. Grasshopper provides a common thread here between A Grammar-Based Generative Urban Design Tool Considering Topographic Constraints and the works of the Computational Planning Group, and with careful handling of data could link processes and scripts from between these two projects, as well as the wealth of other Grasshopper scripts within the overall body of knowledge.

With regard to Public Places, Urban Spaces and the generative design precedents, the literature studied stresses the importance of the urban grid system as either an inevitability of organic growth or a goal to work towards to ensure good connectivity. As a result, this is the logic that will become central to the tool: applying the logic of the urban grid to terrain that cannot usually support a rigid grid structure. 


\section{auters \\ Urban Logics vs Landscape Logics}

With literature supporting the use of a grid logic in tool development, examining real world precedents can verify that this is the correct approach. Continuing to focus on Wellington,

this chapter contains graphic analyses of

Wellington that highlight the logic behind the

growth of Wellington's urban network and

suggest geographic factors that affect the city's

contemporary issues.

The chapter then continues with a photo journal which examines a spectrum of hill suburbs across Wellington in the context of the urban typologies presented in A Grammar-Based Generative Urban Design Tool Considering Topographic Constraints (Rahimian et al. 2019). The combination of graphic and qualitative analyses will verify if the selected approach to tooling is correct. 


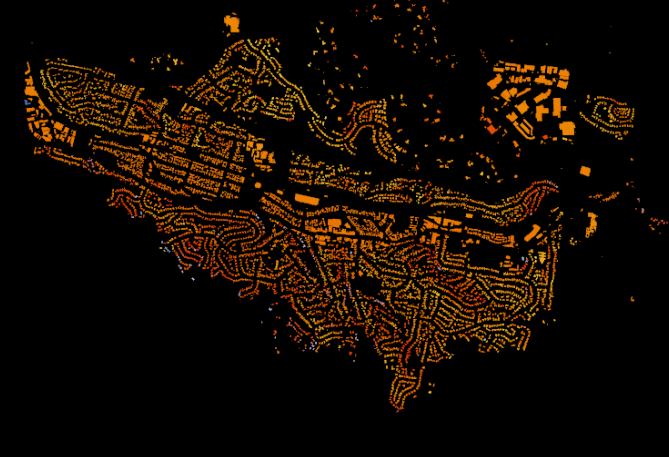

\section{Building Footprint \\ Solar Analysis}

Lowest to Highest Solar Exposure

While always a concern in the discipline of architecture, solar exposure is of particular interest when building on hillside sites. In the southern hemisphere, buildings on south facing hillsides will receive considerably less sunlight than those built on hillsides with other orientations or flat land.

Wellington buildings need to pay particular attention to this due to the damp and humid climate the city experiences for much of the

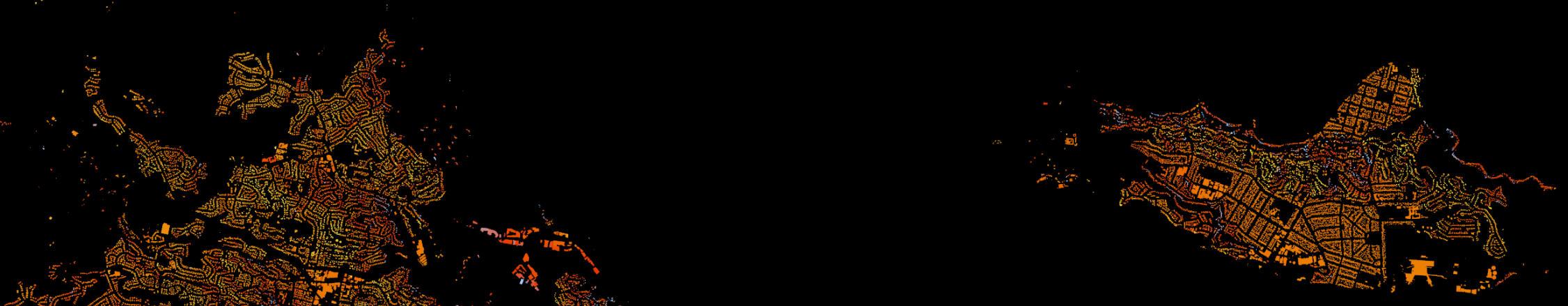

year. While a complex multifaceted problem, the city's endemic damp or mouldy homes problem is certainly prevalent in areas that receive comparatively less sunlight. One such example is Aro Valley, where $40 \%$ of houses were found to be damp some or all of the time (Mckenzie 2020). It may be beneficial for the tool to have inbuilt solar analysis capabilities so that design choices can be made around whether proposed sites receive enough sunlight.

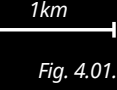




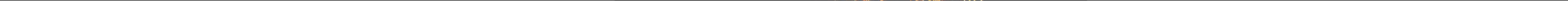



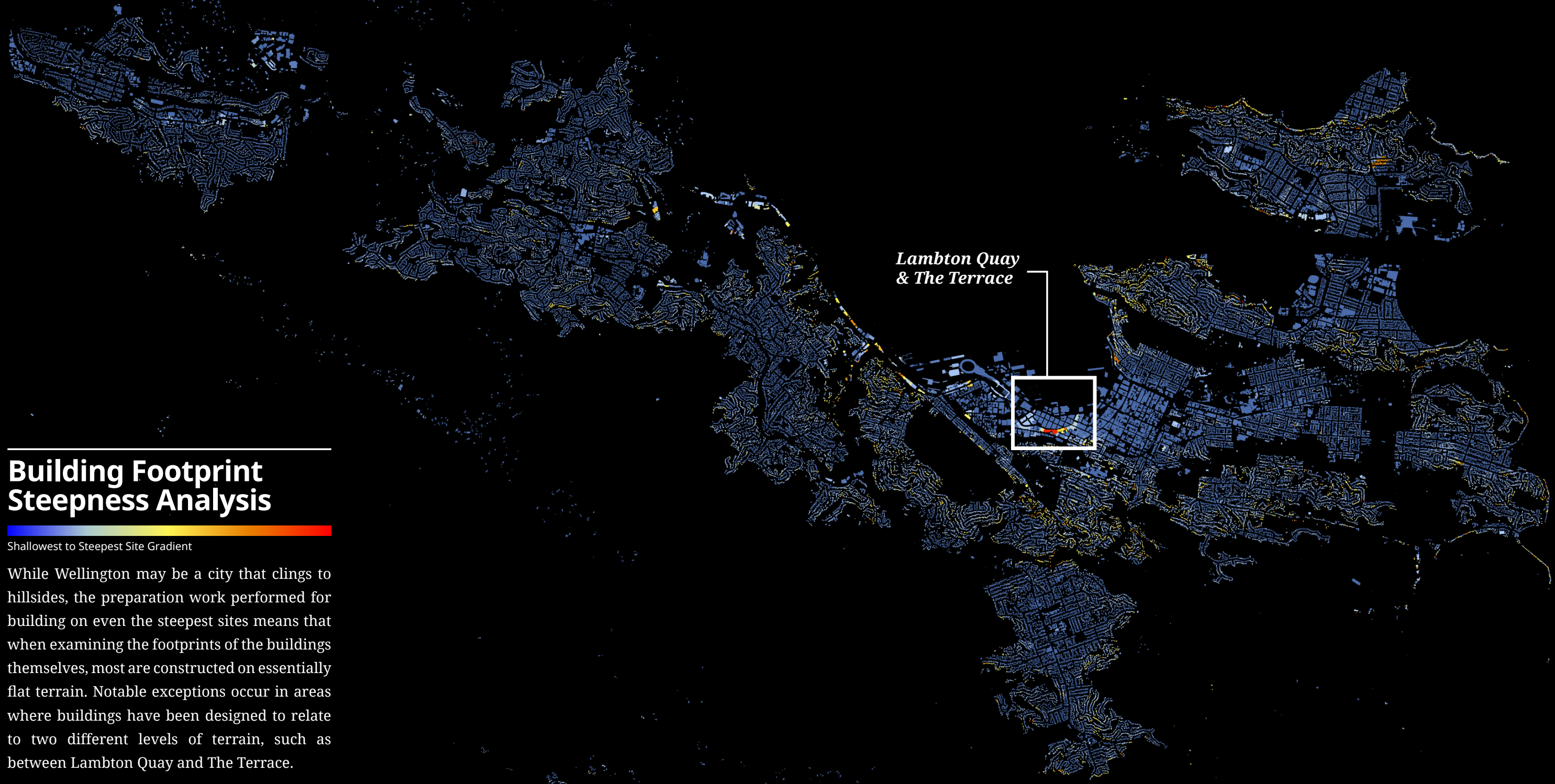

between Lambton Quay and The Terrace. 


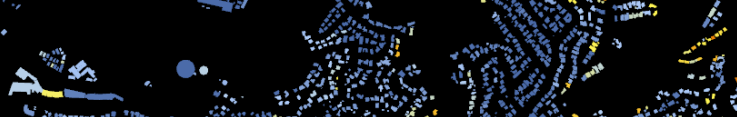
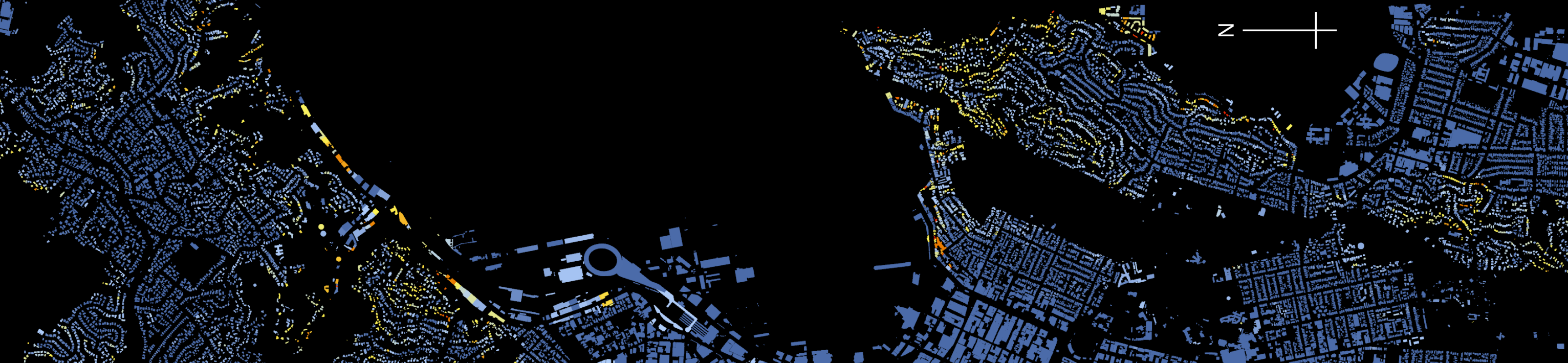

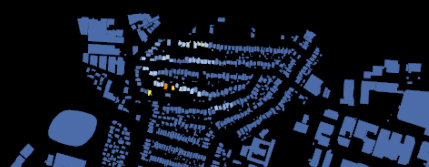

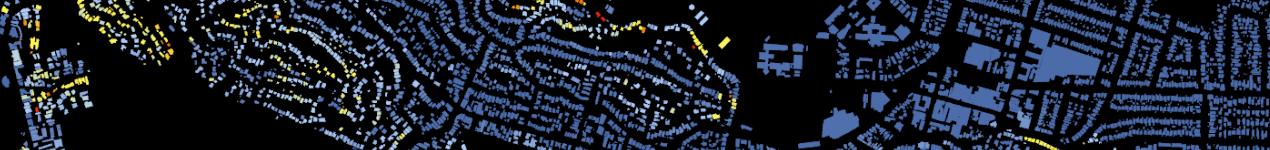
1. 1. 30<smiles>C=[Tl]</smiles>
n * 10 mo

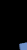

\section{Building Footprint}

\section{Steepness Analysis}

mithon

\%

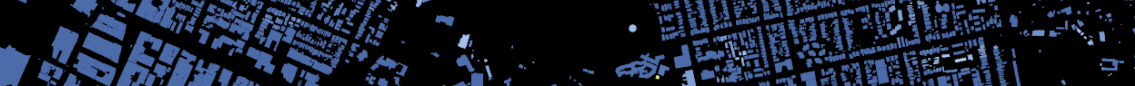

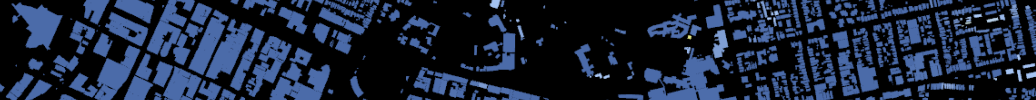

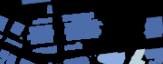

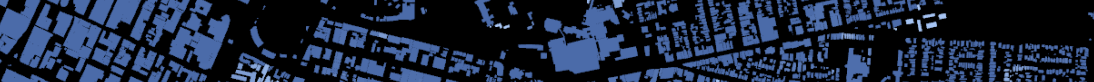
$=1, y$

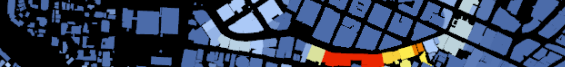

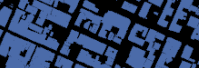

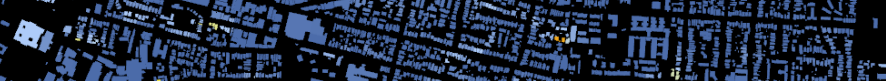

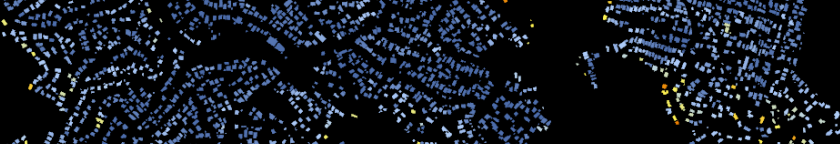

1.

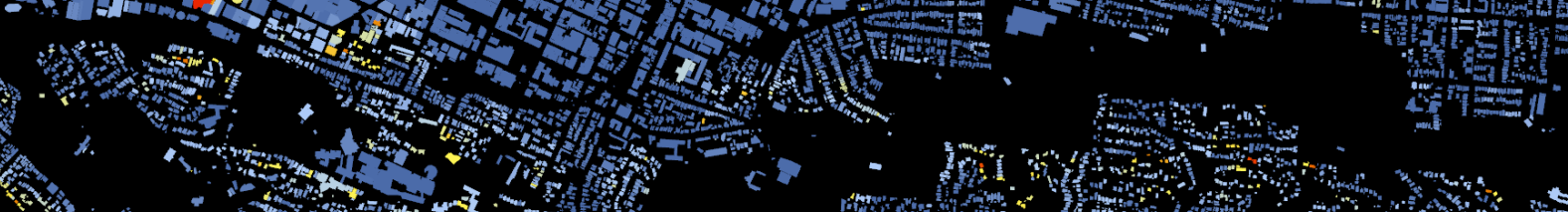

3.

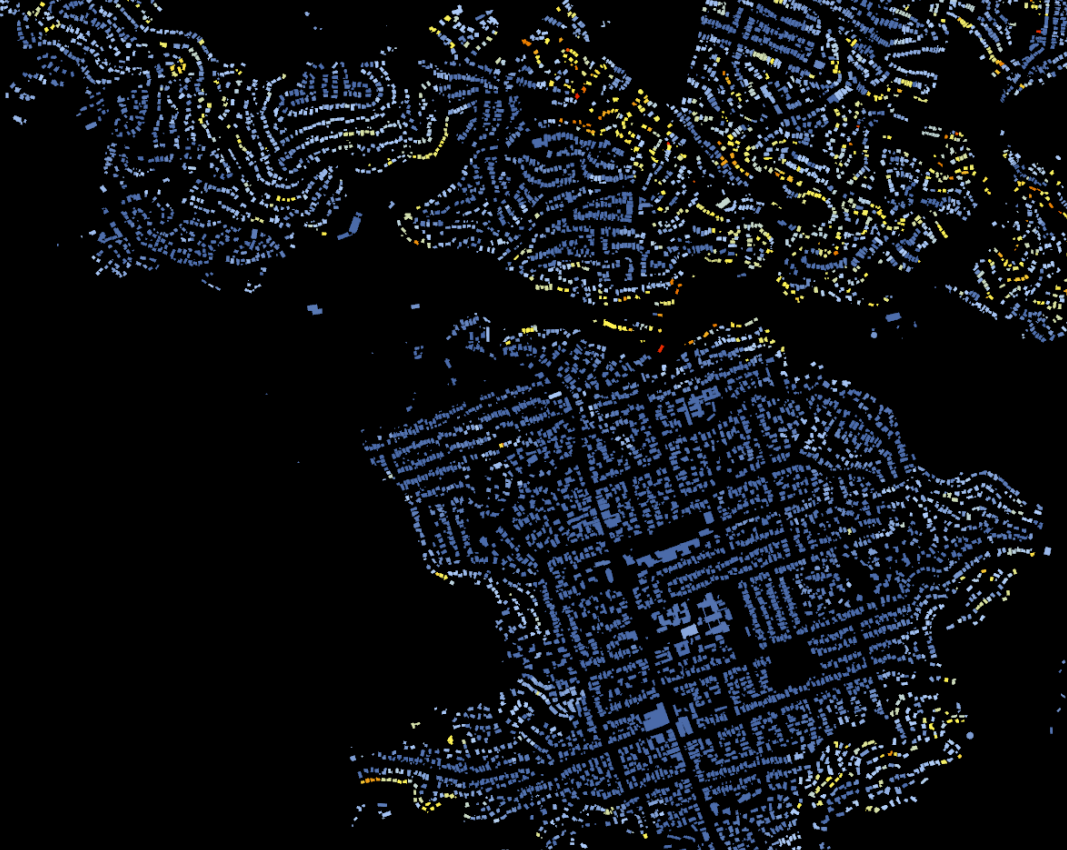

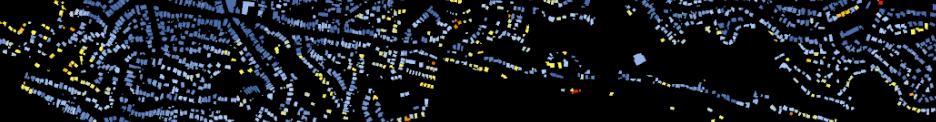
(3)

Shallowest to Steepest Site Gradient 

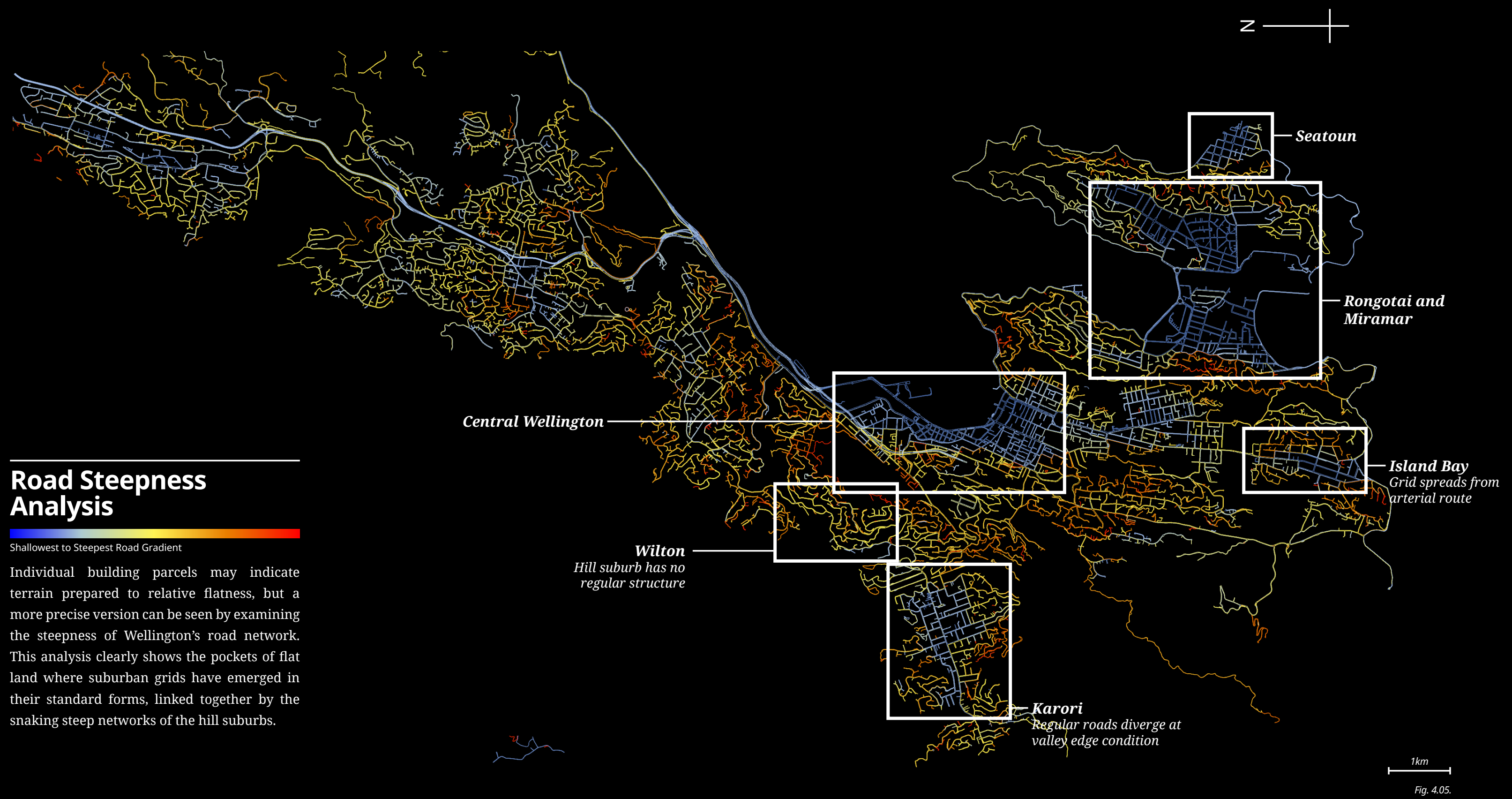


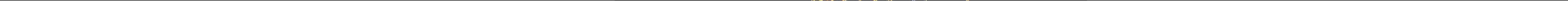




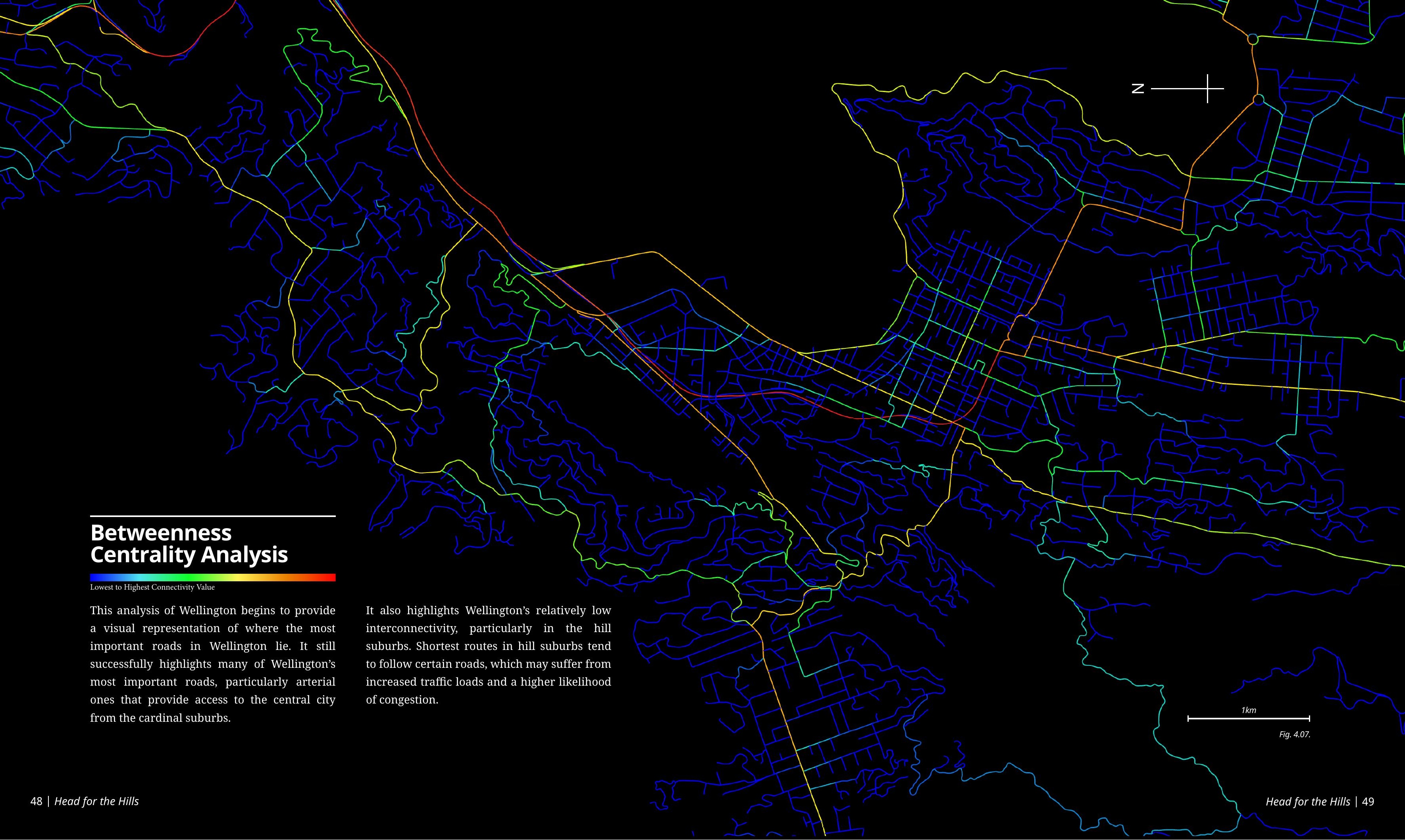




\section{Wellington \\ Urban Typologies \\ Photojournal}

To reiterate, Beirao and Duarte (2019) found three typologies of the urban grid around sloping topography: organic grids, orthogonal grids, and mixed grids.

This content is unavailable. Please consult the figure list for further details.

Fig. 4.08. The typologies of the San Diego Urban Network.

As this thesis is focusing on how to bring rational order to the steepest terrain, the concept of the organic grid is the most relevant. Using urban schemes around Wellington as precedents, the three typologies can be reclassified into 6 typologies:

- Standard Urban Grid

- $\quad$ Face Adjusted Grid

- The urban grid can continue beyond slopes of $15 \%$ but only makes sense if the orthogonal roads are exactly perpendicular or parallel to the landscape

- Face Adjusted Parallel

- 'Parallel' in this case describes when the road system mostly follows parallel to contour lines, moving between levels only when the terrain is at a suitable grade
- Grid-to-parallel edge condition

Typically the edge between the two systems will be at the base of the hill, similar to the $15 \%$ slope edge in Beirao and Duarte (2019)

- Ridges and Valleys

- This is most similar to the Organic Grid (Ibid), but describes a more arterial system rather than a grid system

\section{Landscape Adaptive}

- This typology occurs in more freeform terrain where the key ridges and valleys are less prominent. Small topographical features dictate cul-de-sacs and narrow access roads for maximum site usage

These typologies begin to inform what logic will be coded into the tool. In order to understand how these typologies relate to the built vernacular that the terrain necessitates, brief photo journals were created for each. The suburbs chosen as study areas are varied in density, age and demographics. The 'density' denoted to each suburb is relative to the suburbs selected Wellington has very few areas that are 'high density' in the commonly accepted use of the term, and all of them within the central city and on flat land.

Included in the photo journal is a plan view of the relevant typology and a simple betweenness centrality analysis. This analysis gives a graphical representation of where the important roads in a network lie and how they relate to the overall system.
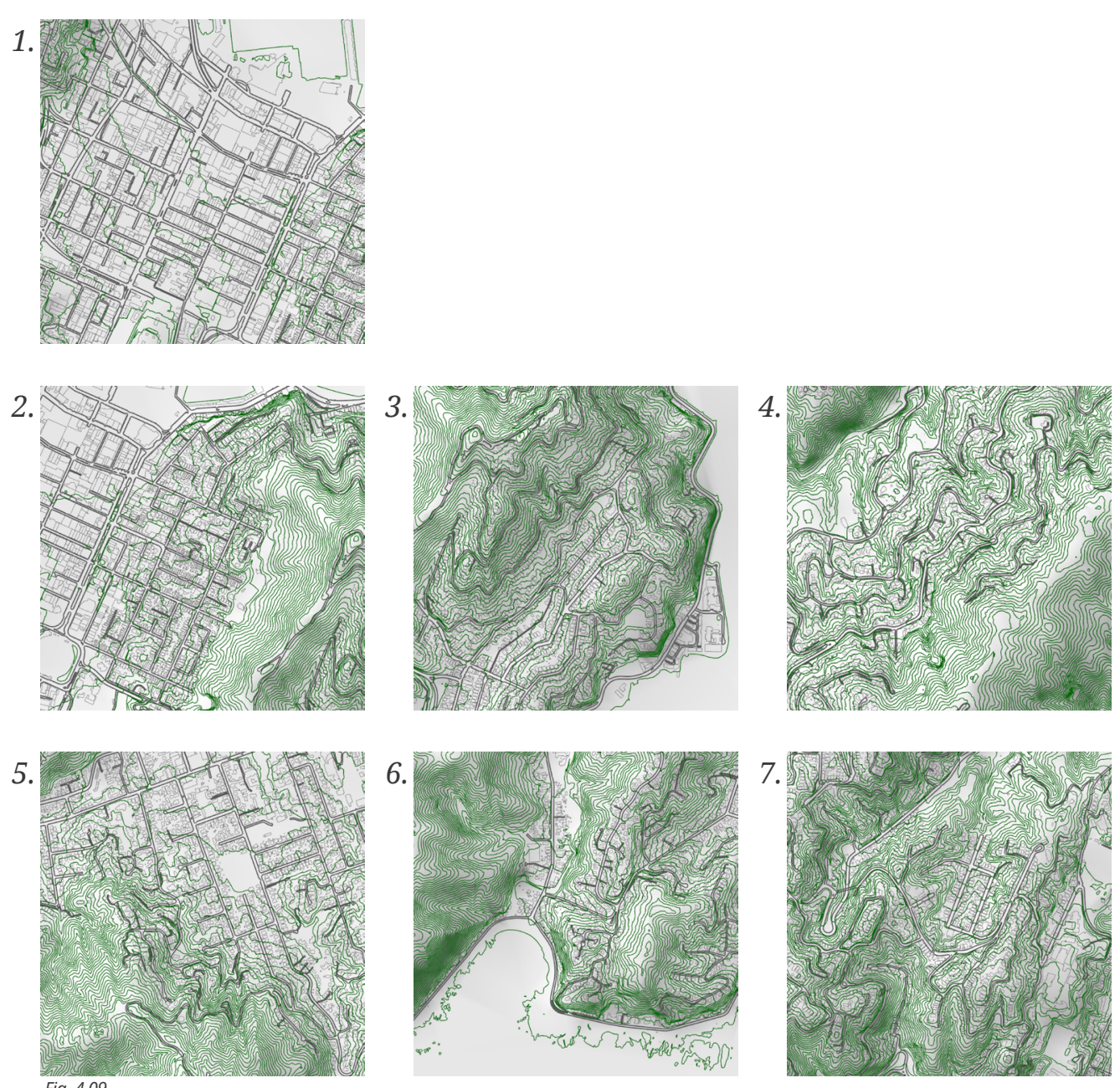

1. Standard Grid: Te Aro

2. Face Adjusted Grid: Mt Victoria

3. Face Adjusted Parallel: Hataitai 4. Face Adjusted Parallel: Wilton 5. Grid-to-parallel Edge: Karori

6. Ridges and Valleys: Owhiro Bay 7. Landscape Adaptive: Kelburn 

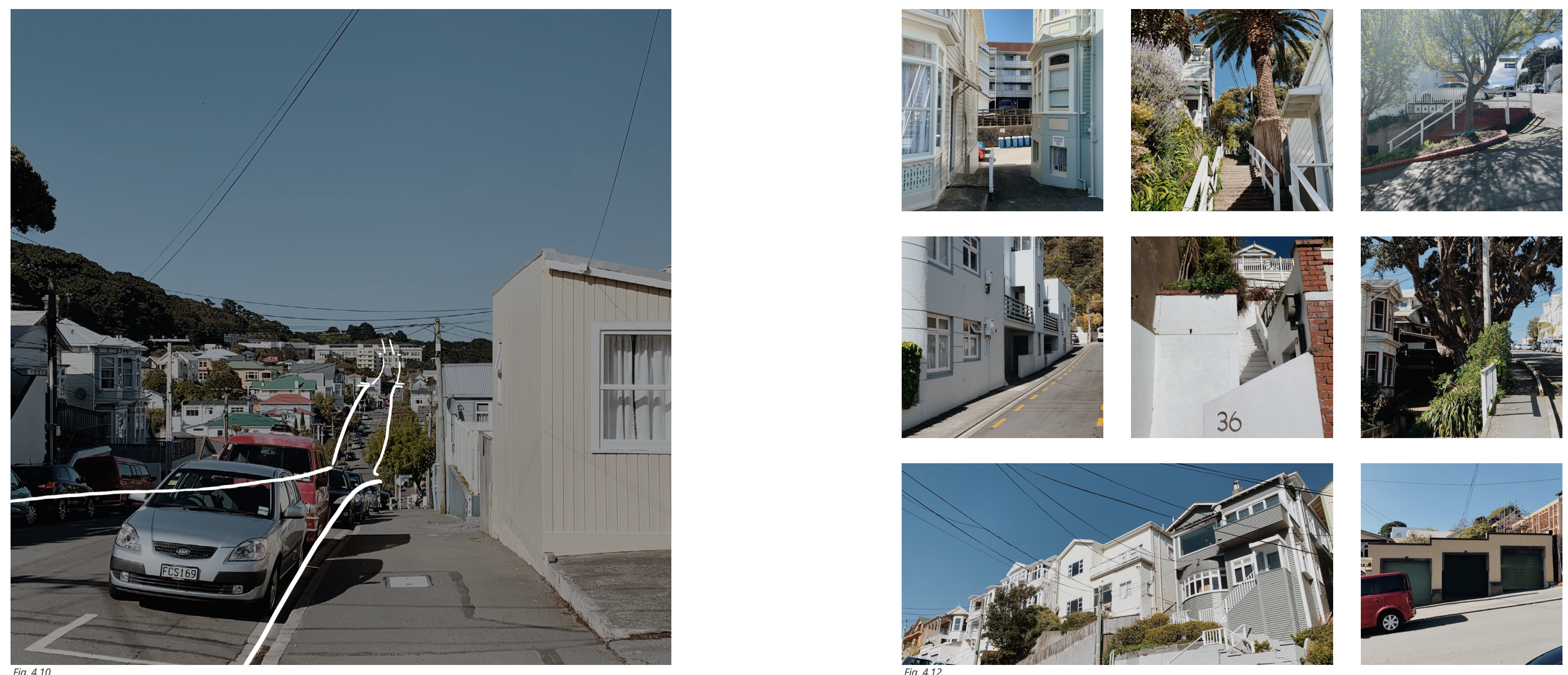

\section{Mt Victoria}

Fypology

Density

1840

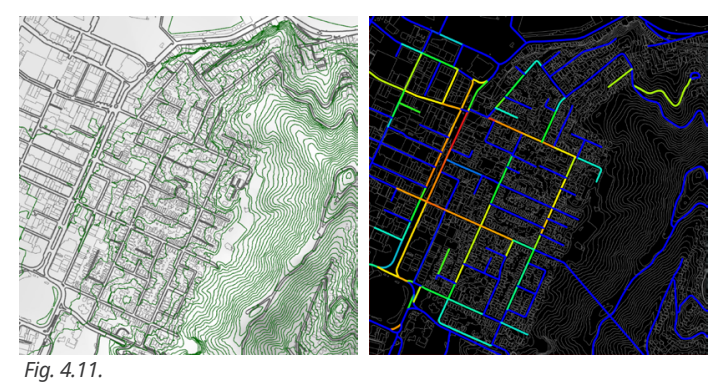

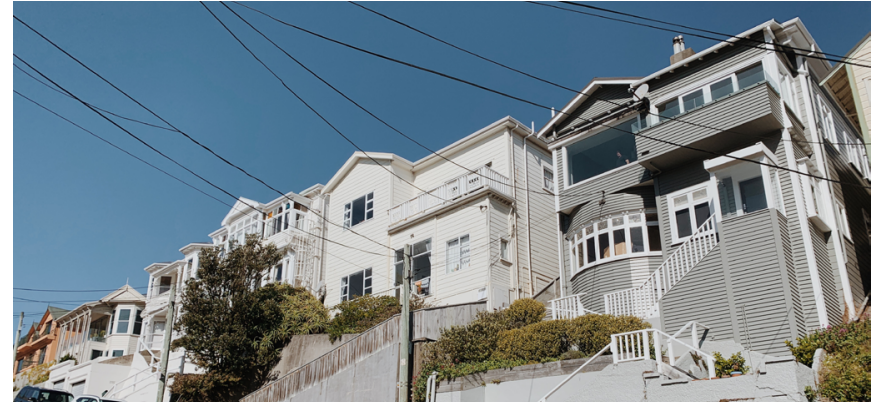

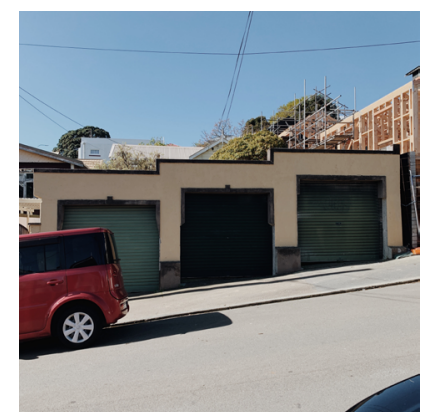

The regular grid of Te Aro remains intact as it meets the base of Mt Victoria, climbing until it meets the Town Belt. Towards the north, the

grid is warped around the curvature of the landscape before becoming a parallel typology towards Oriental Bay.

The landscape is not completely even, meaning that the roads parallel to the fall line of can occasionally be very steep. The average steepness of these roads throughout the suburb is $9 \%$, with the most extreme segment being in Scarborough Terrace at 23\% (Hunt 2019). 

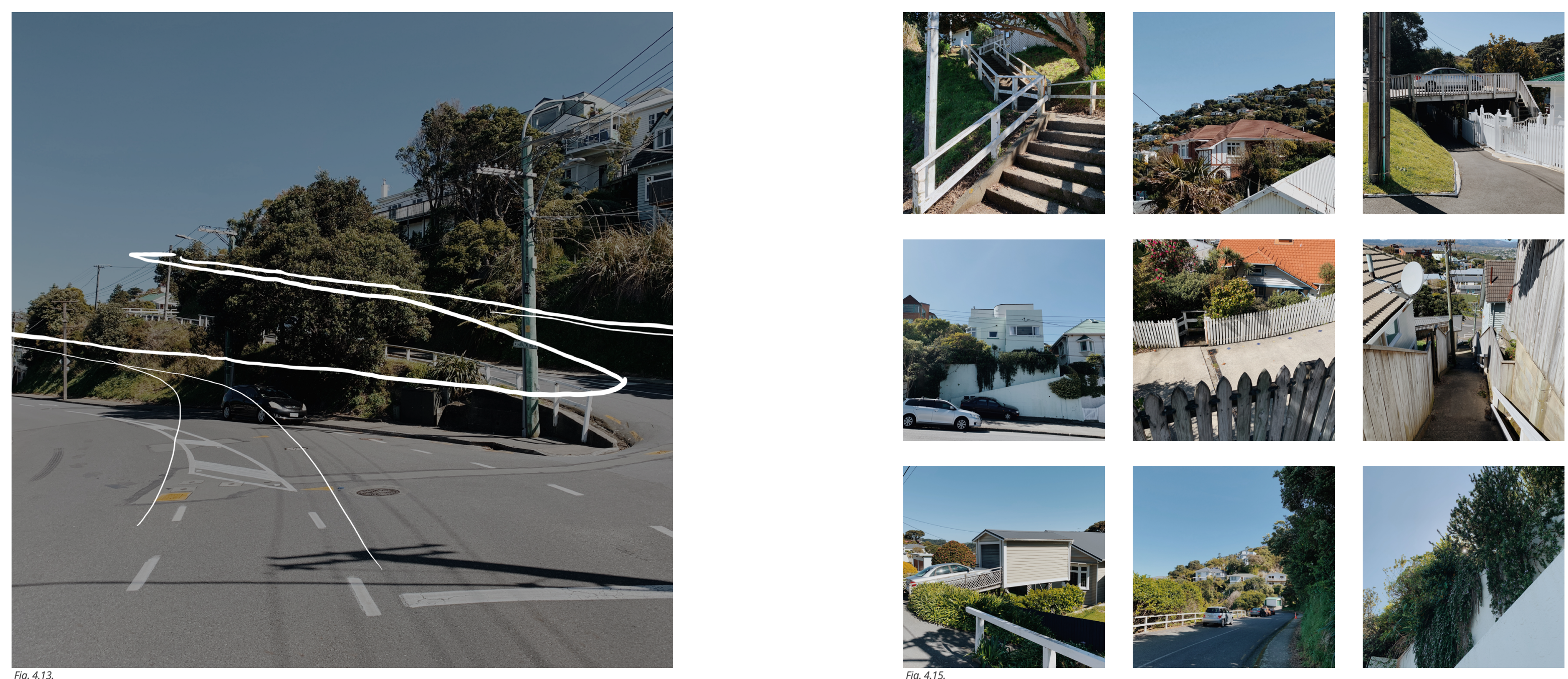

\section{Hataitai}

Fypology

Density

1902

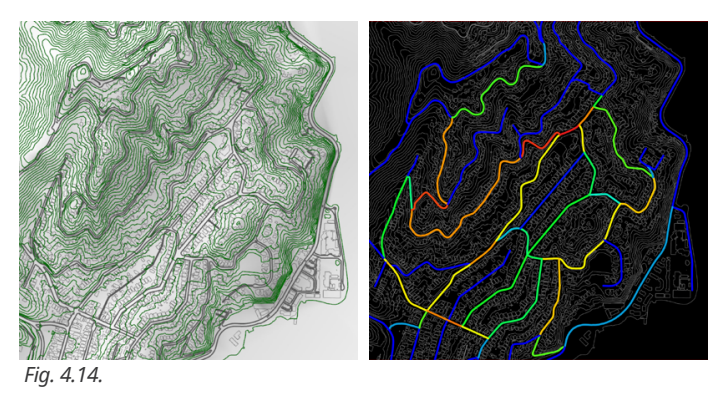

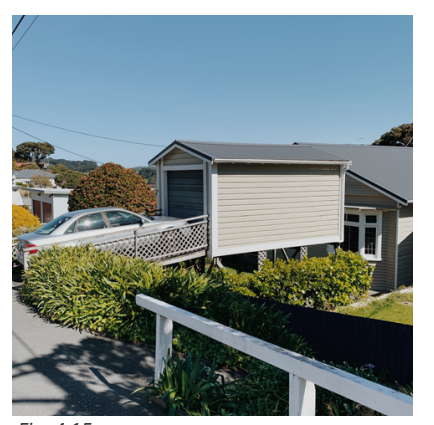

Hataitai developed from rural farmland into lifestyle blocks for wealthy settlers before

adopting its suburban morphology. Roads

developed organically from farm tracks,

resulting in the contour-parallel configuration

of the suburb seen today (Kebbell and Ombler

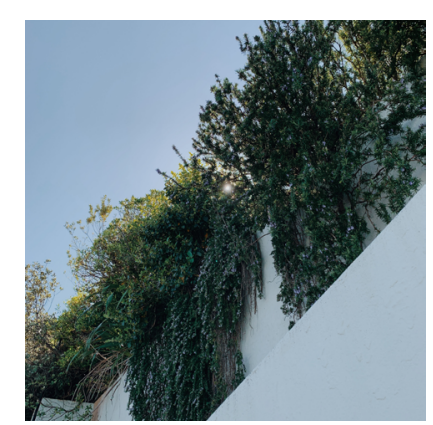

018). Circulation between these roads is provided by limited pedestrian staircases, though access to houses is often provided by private staircases that could possibly be linked to complete a cross-block access (Dalley, 2020) 

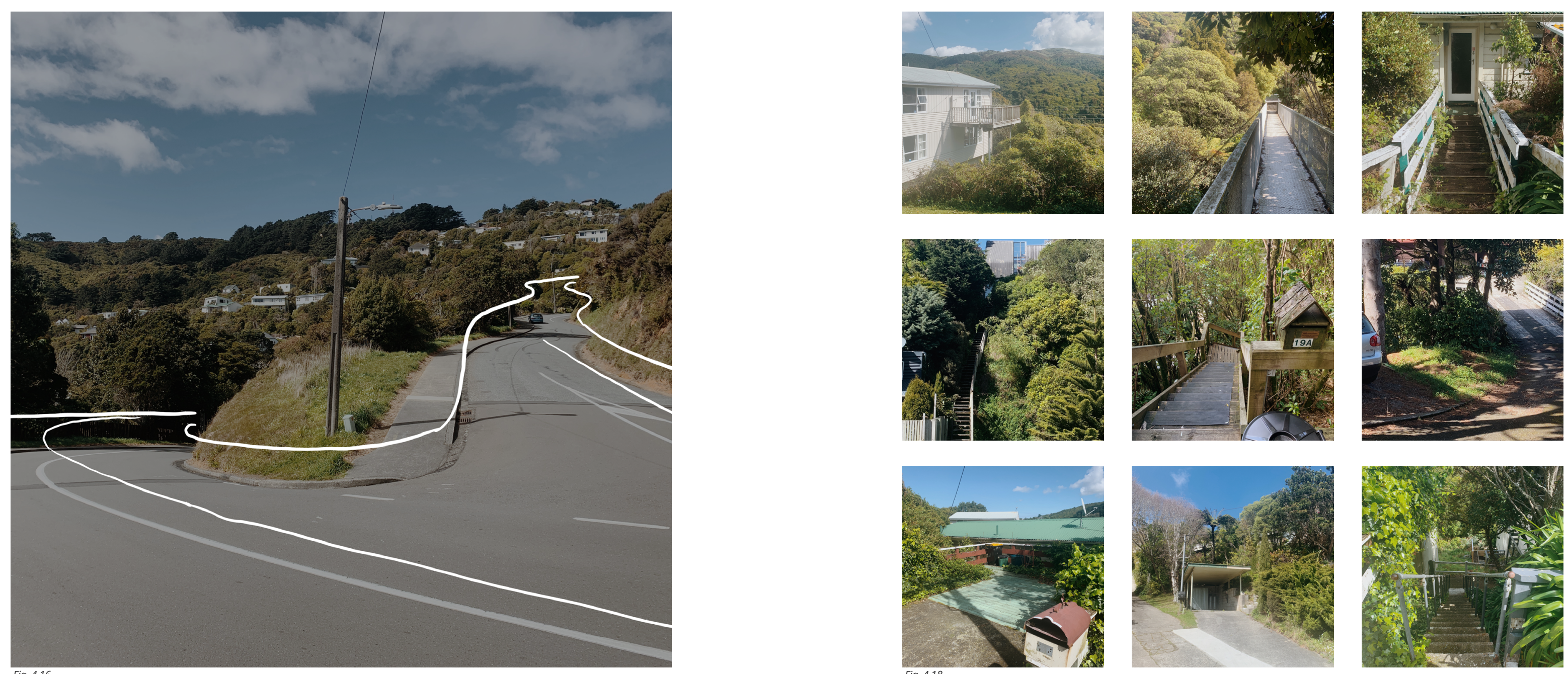

\section{Wilton}

Fypology

Low

Start of Development

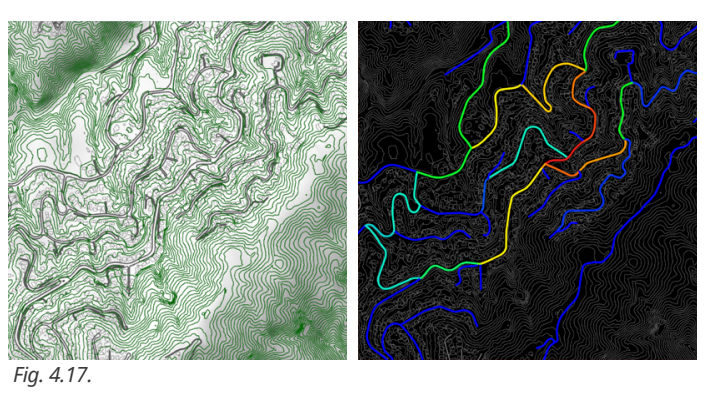

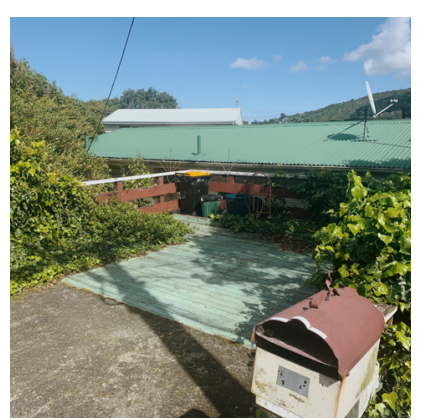

As a suburb further from the centre of the city and younger than the previous two, it comes

as no surprise wilton fits the definton o

modernist urban space with buildings reading

as individual "objects in space" (Carmona et

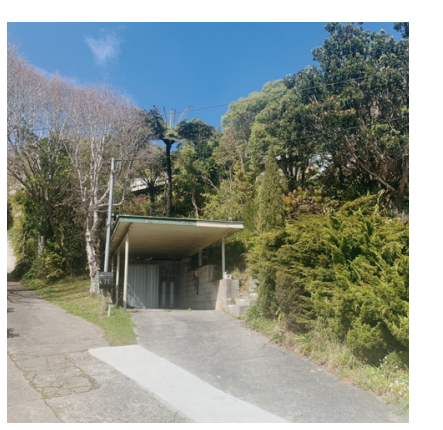

al. 2003). Some buildings utilized bridges to circumvent unique conditions in the terrain, in order to access sites across a stream from a main road or to access the second floor of a building directly from street level. 

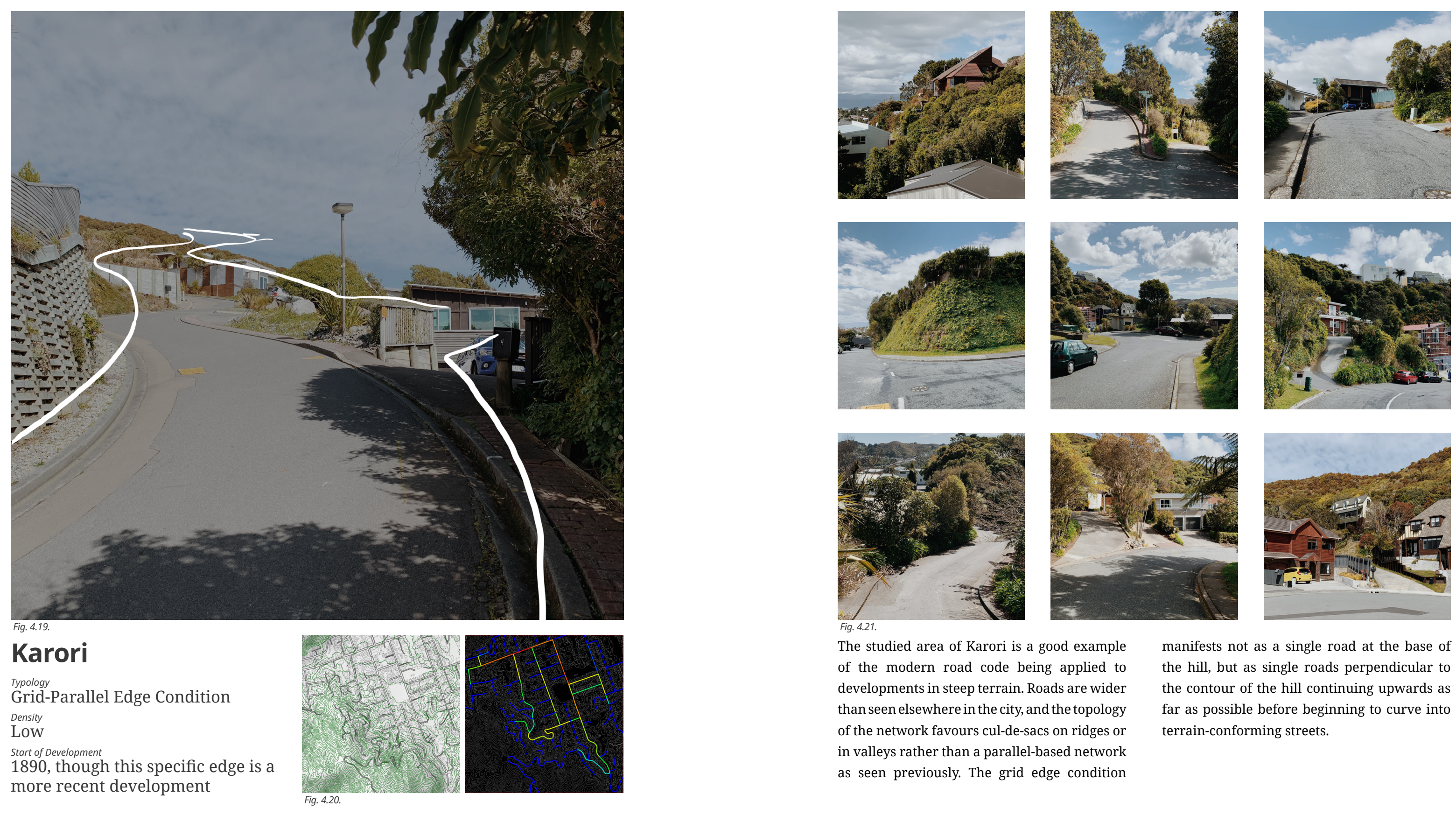

The studied area of Karori is a good example of the modern road code being applied to developments in steep terrain. Roads are wider

than seen elsewhere in the city, and the topology of the network favours cul-de-sacs on ridges or in valleys rather than a parallel-based network

manifests not as a single road at the base of the hill, but as single roads perpendicular to the contour of the hill continuing upwards as far as possible before beginning to curve into as seen previously. The grid edge condition 

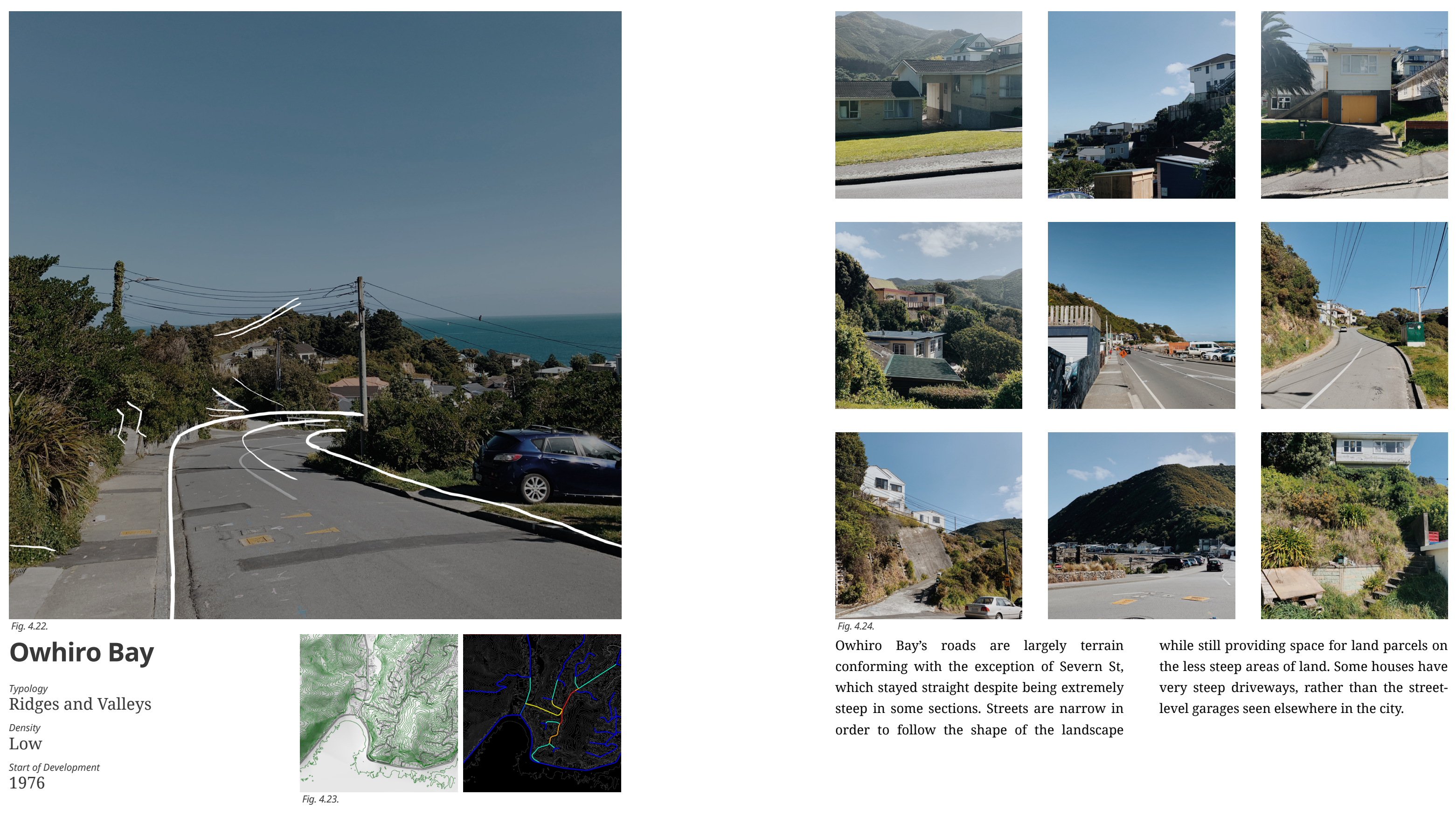

Owhiro Bay's roads are largely terrain

conforming with the exception of Severn St,

which stayed straight despite being extremely

while still providing space for land parcels on

steep in some sections. Streets are narrow in tess steep areas of land. Some houses have very steep driveways, rather than the streetorder to follow the shape of the landscape 

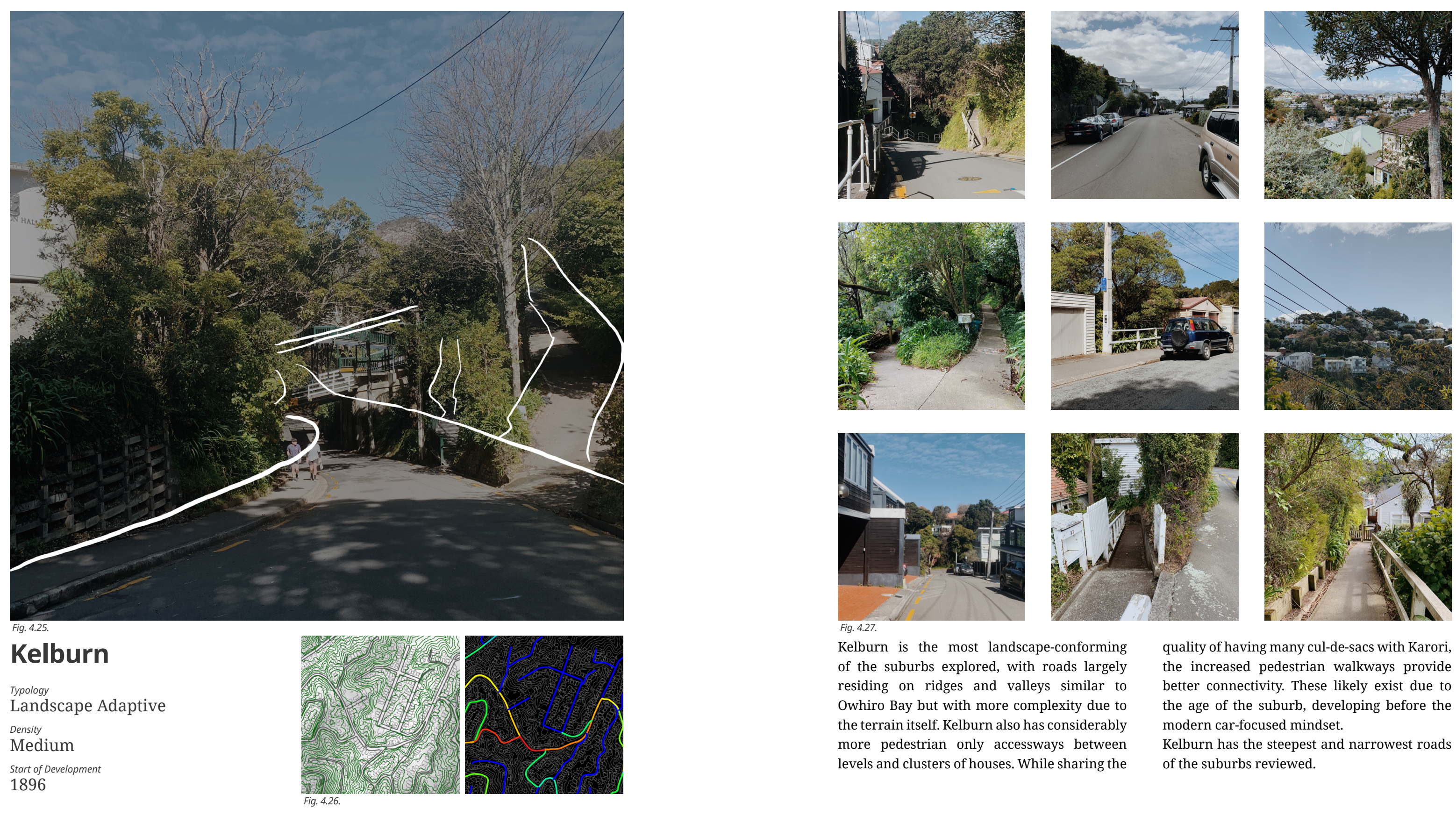

Kelburn is the most landscape-conforming of the suburbs explored, with roads largely

residing on rides and valleys similar

residing on ridges and valley

plexity due to

the terrain itself. Kelburn also has considerably

more pedestrian only accessways between

quality of having many cul-de-sacs with Karori, the increased pedestrian walkways provide better connectivity. These likely exist due to the age of the suburb, developing before the levels and clusters of houses. While sharing the

modern car-focused mindset

Kelburn has the steepest and narrowest roads of the suburbs reviewed. 
The key conclusion that can be drawn from the photo journal is the incredible resilience and adaptability of Wellingtonian housing design. Across all these suburbs, houses above hug retaining walls or improbable garages on stilts flank the narrow roads that negotiate the hilly terrain.

There are three different kinds of site configuration possible when building on grade: uphill, downhill and flat (Levin 1991). Uphill and downhill sites refer to where the building platform is located in relation to its access road, and flat sites are ones that have had earthworks done to remove the grade factor.

In Wellington the uphill and downhill typologies are distinctive all the way up to their intersection with the road: car storage presents a regular but difficult challenge for houses

on the winding hillside roads. In the steepest suburbs surveyed, cars are parked on platforms that are either a simple deck or full garage structures on downhill sites.

On the uphill side, garages tend to be dug into the hillside, either forming a basement for the house or sitting underneath the level of the house's front yard. Car storage remains an important issue to tackle as Wellington is currently a highly car-focused city. The viability of any new development requires infrastructure intensification not just on the site itself but within the network that would connect it to the central city.

In the suburbs with higher density, there is more of a tendency to build right up to the site boundary particularly on uphill sites. Uphill sites where the road was considerably

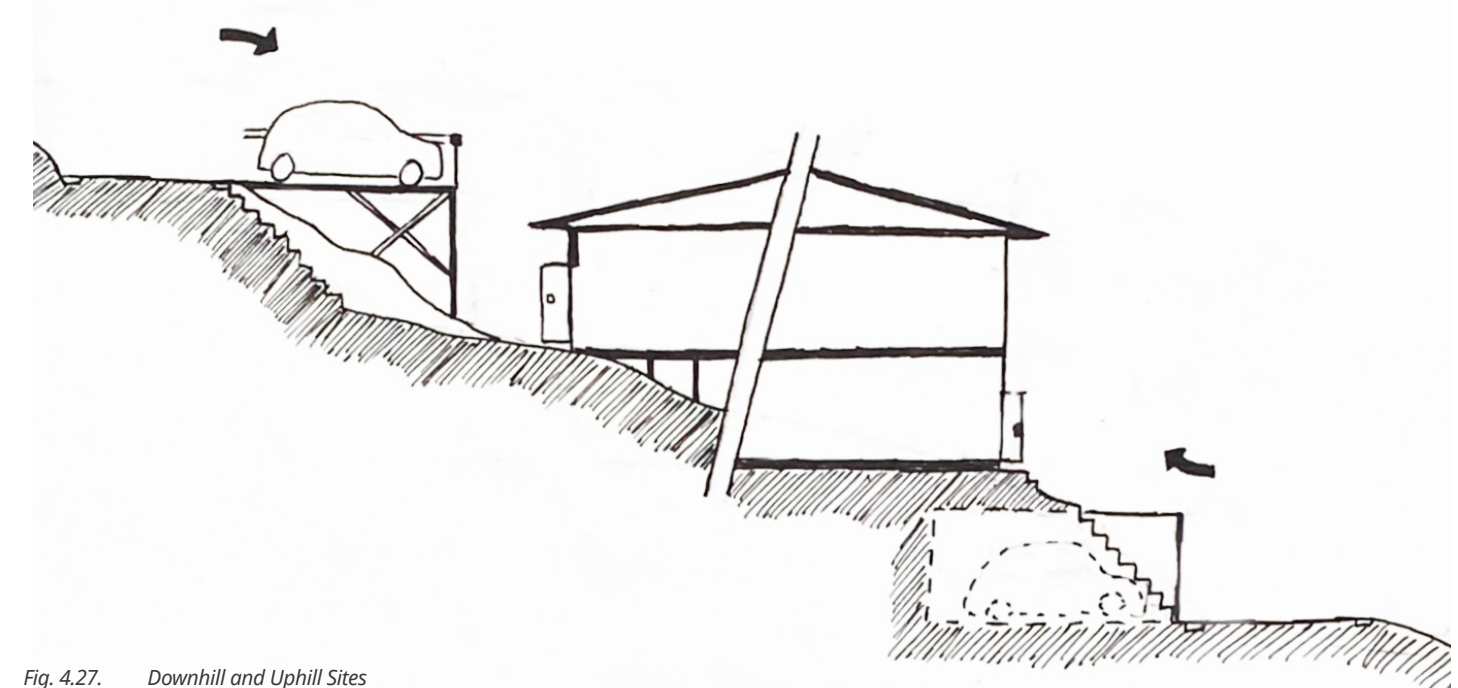

lower than the building platform often have retaining walls which give the impression of the built fabric starting at the street boundary itself. The greatest impression of verticality occurs when the suburb fits Carmona's definition of "traditional urban space", with buildings embedded in block and not serving as freestanding objects in space (Carmona et al. 2003). On the steepest sites 2 storey villas became essentially 4 storeys high from street level, after their garage and retainin wall levelled the site for construction. Thes buildings form a manmade landscape that sits soundly within the terrain and give a strong impression of the landscape's articulation.

Now that it is established that the built fabric is capable of adaptation to terrain in both traditional and modernist space morphologies, the spatial layout of the suburbs can be considered. The different urban typologies have different levels of connectivity within them, and to varying degrees of success. The specific landform of each suburb determines whether the network is one with a grid, with limited connections between bands of roads, or is a series of cul-de-sacs. The qualitative feel of the area is affected greatly by this well connected areas feel more lively and have more pedestrians, whereas cul-de-sacs (particularly in the large new developments in Karori) are unwelcoming. While cul-desacs enhance the privacy of houses, current urban thinking frequently decries them as "the isolated, insular, private enclave, set in formless sprawl of similar enclaves, separated socially and physically from the larger world, and dependent upon the automobile for survival" (Southworth \& Ben-Joseph 1997). The photo journal supports the decision from the literature review to proceed with a grid logic as the main driver of the tool.
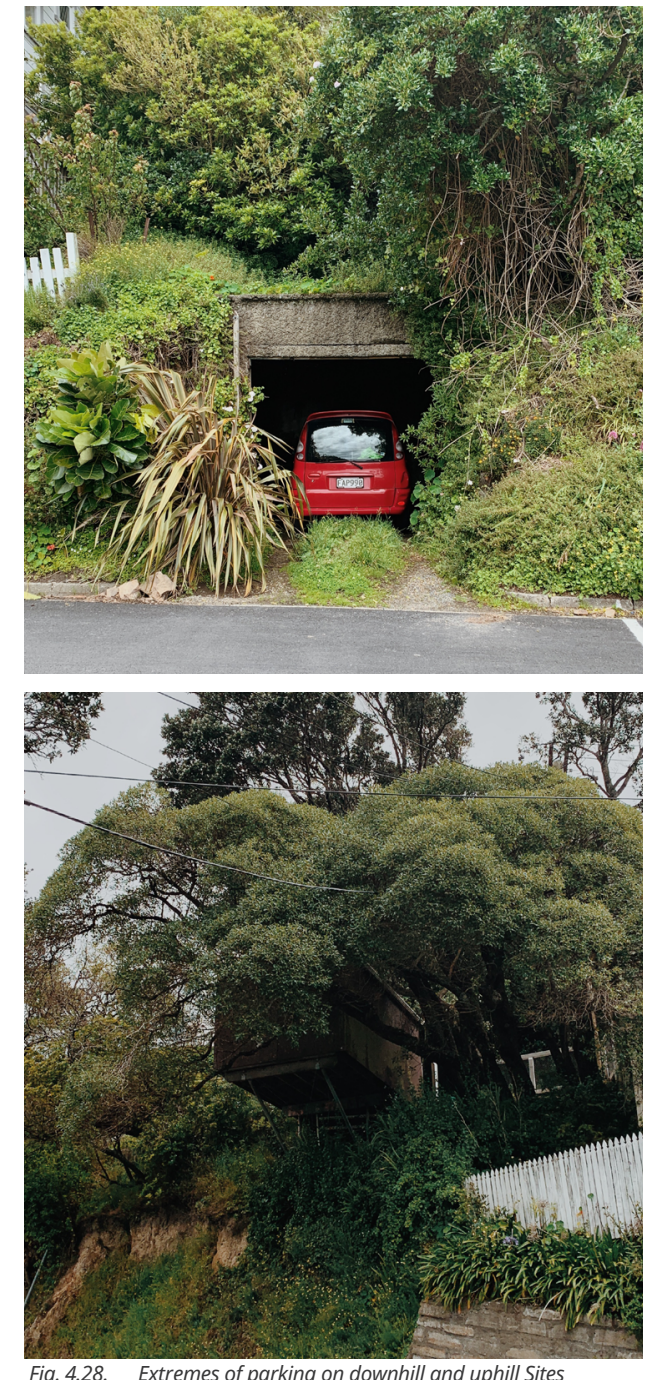


\section{Site}

In order to serve as a consistent base for development of the tool and an opportunity to produce a proof-of-concept scheme, a Wellington site has been selected to use throughout this research.

The selected site is a valley between Karori and Makara currently used as a forestry site. This will be referred to as North Makara Valley, as there are no named features in this valley other than North Makara Stream (NZGB 2013).

This site was selected among considered Wellington sites for having properties that would suit the tool's development but challenge its output: a variety of terrain steepness, an entire valley of land area that will experience different solar conditions, and a potential for connection to existing urban networks.

Were this site to be pursued as a future development it would require a comprehensive infrastructure upgrade in Karori and Kelburn to enable residents to travel into the city. Thi planning is outside the scope of the thesis and will not be undertaken as part of this research.

In order to further demonstrate the tool, after the step by step record of the proof-of-concept scheme, a series of other outputs from the suburbs studied in the photo journal will also be included.

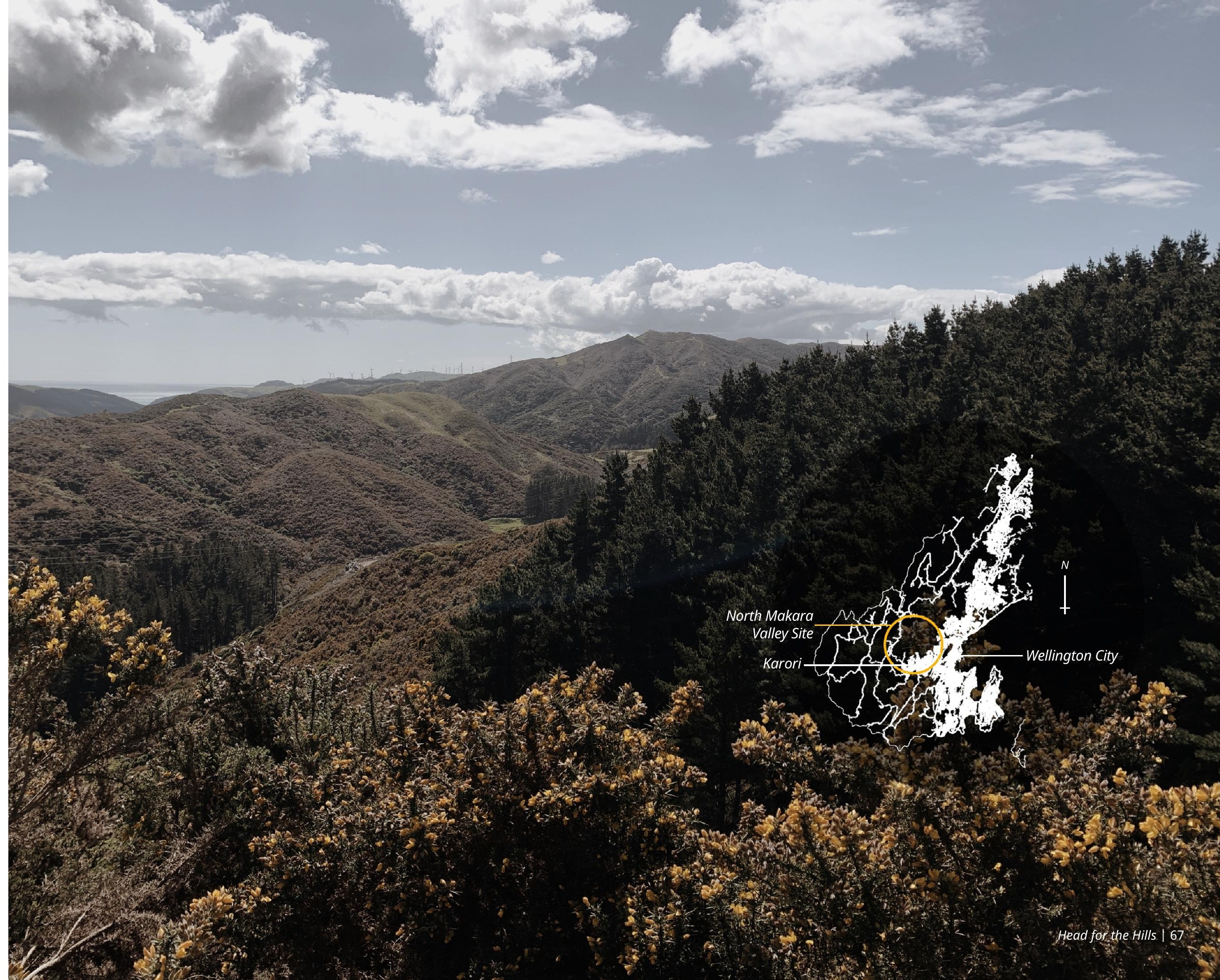




\section{Chapter 5 \\ Prototypes}

This chapter describes a series of prototype

scripts designed to fill the six purposes outlined

within the methodology diagram. They are

presented here in the order they were created,

which roughly corresponds to the workflow

suggested by the methodology. 


\section{GIS Modeller}

Geographic Information Systems (GIS) are "frameworks for storing and analysing spatial information” (Belesky 2018). GIS is commonly used by city and regional councils as a way of maintaining a publicly available database of information about the geography of their jurisdiction. This can range from simple topographical information like contour lines or streams to infrastructure like roads or drainage, all the way to human-imposed regional boundaries like the borders between electorates or land use areas. For this thesis the focus is on the topographical information of the site.

The inputs of this definition are GIS files describing contours, building footprints and road kerbs, which were obtained from the Wellington City Council and Greater Wellington Regional Council websites. These files were collated using the open source GIS program QGIS (QGIS 2020) to ensure the maps overlaid properly and had the same map projection. A boundary curve also needs to be drawn by the user to determine how the topography is cropped before creating the final mesh.

The plugin used in Grasshopper to interpret the GIS Shapefile (.shp file) is GHSHP (Hiteca 2019).

Contour lines are put through a simple series of components. After being cropped to conform with the boundary, the lines are joined, then divided into points at regular intervals along the length. The distance between intervals is configurable, allowing the user to control the resolution (complexity and accuracy) of the final mesh. These points are then put through a Delaunay connectivity (Delaunay 1934) component, which connects close points in groups of 3 to create the triangular faces which compose the mesh.
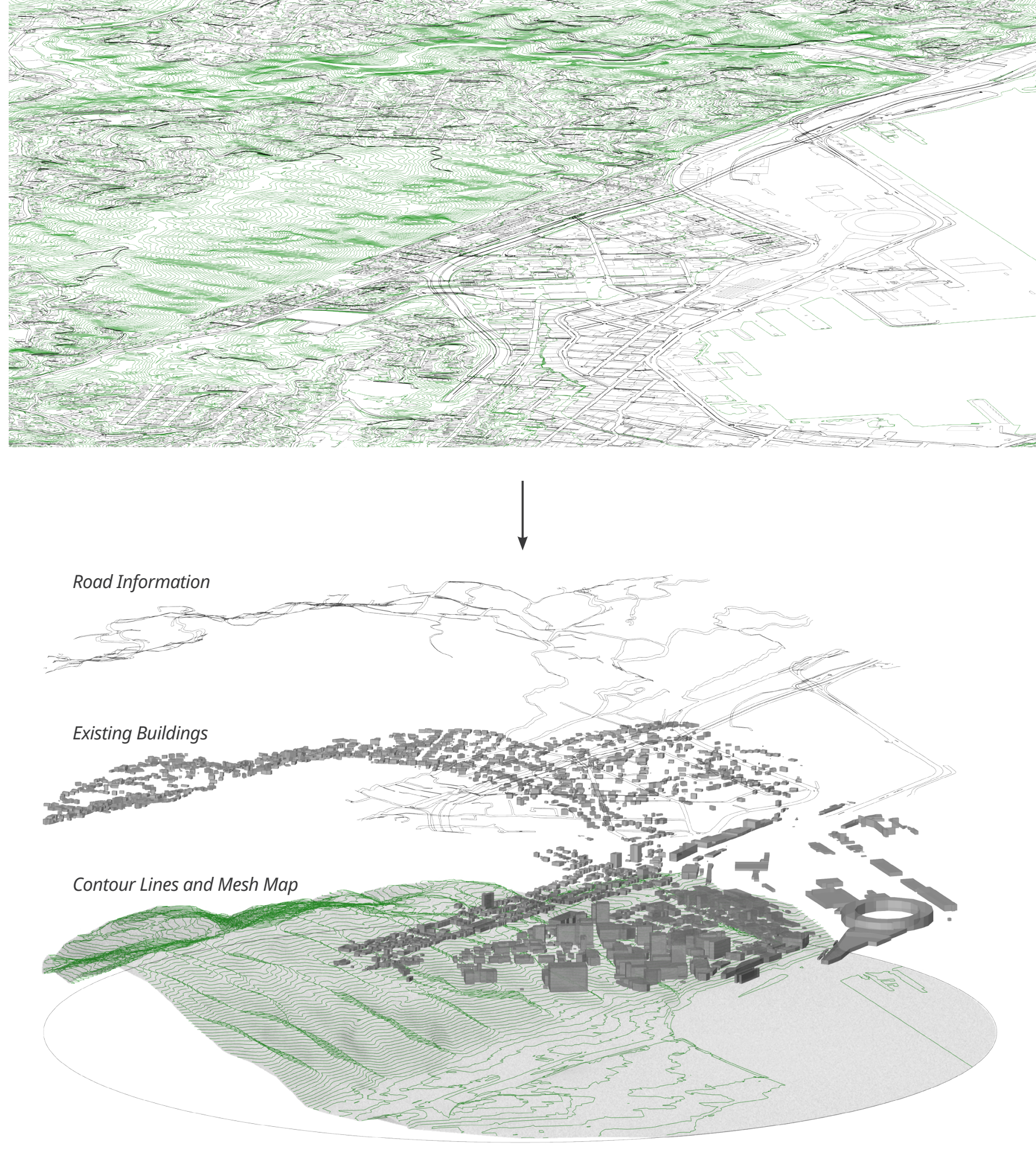


\section{Mesh Analysis}

Grasshopper is able to easily translate information in a mesh into coloured representations in order to highlight certain qualities of the mesh. For the purposes of this thesis, certain qualities of the terrain are likely to be valuable in driving site decisions. Three such analyses are a height analysis, steepness analysis, and solar analysis.

\section{Heightmap}

The terrain mesh in Rhinoceros is easy to understand three-dimensionally when a user can move around the object. When printed or displayed statically it can be harder to understand. A heightmap is a simple way of colour-coding the mesh based on each point's height. This enables the mesh to have a visual representation of its height, even when viewed in plan. Each mesh vertex is assigned a colour based on its z-coordinate.

\section{Steepness Map}

Another way of understanding a mesh in plan view that is particularly relevant to this thesis is examining how steep the mesh is at any location. The normal vector (a vector perpendicular to the surface) of each mesh face is obtained, and then the z-component of this vector is used to colour the mesh face. This visualisation provides a good way to evaluate areas that may or may not be suitable for development at an early point.
Solar Analysis

As discussed in the urban analysis chapter, it is valuable terrain when deciding on a site to develop. This is achieved using Grasshopper plugin Ladybug (Sadeghipour Roudsari 2013), can simulate the solar exposure of the mesh All that is needed is an .epw file which can be obtained from Energy Plus, an open-source weather data and analysis project.

Using Ladybug's built in functionality, a custom date range can be used for the analysis, or the default whole year. This could be helpful to focus study on winter months when solar exposure is at its lowest.
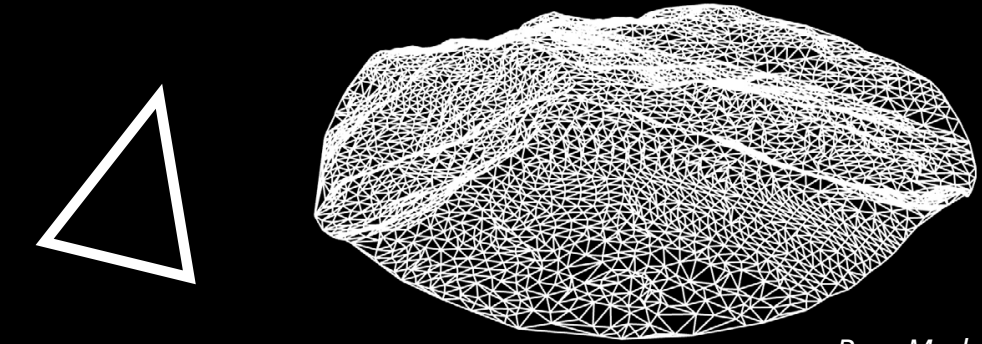

Base Mesh

$z$
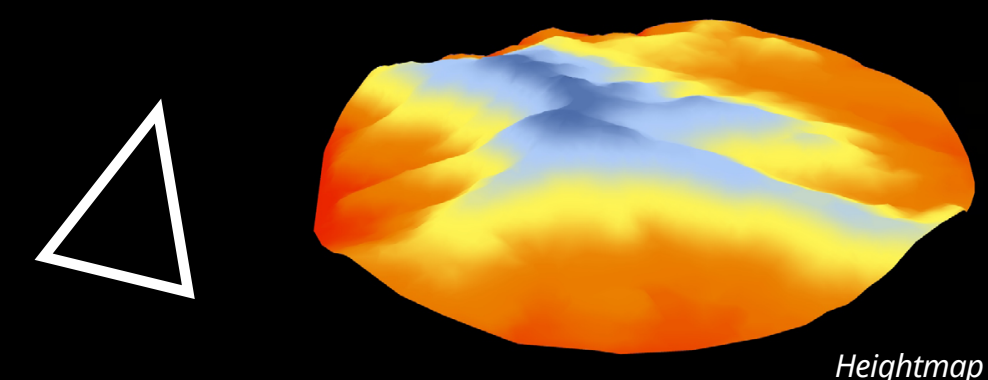

Heightmap
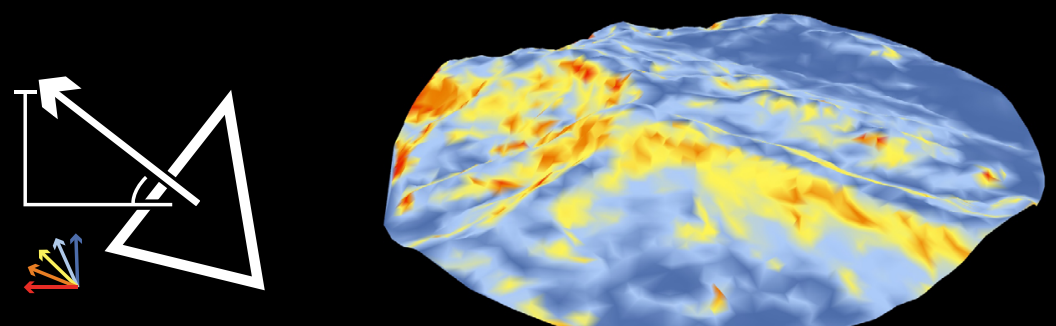

Steepness Map

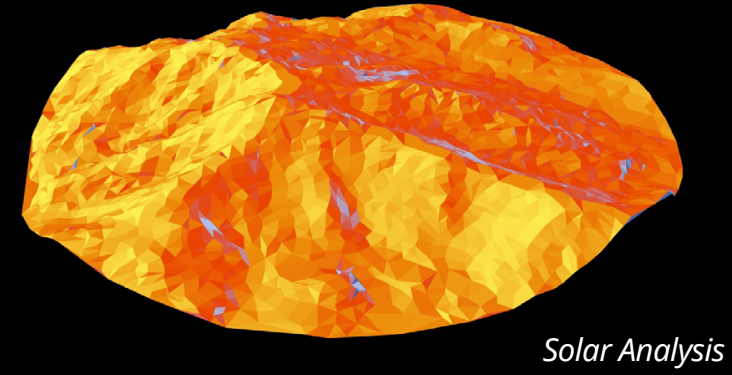




\section{Pathfinder}

The process of negotiating a road up a sloped site is a difficult one, where overly steep slopes limit vehicles that can travel up them. This part of the tool uses a script produced as part of a workshop run by the University of Pennsylvania and PEG Office of Landscape + Architecture (VanDerSys 2017). The script uses the Anemone plugin (Zwierzycki 2013) to enable parts of the definition to loop, allowing the simulation of an agent moving over the terrain and evaluating the direction it should proceed in with each step towards a goal.

The agent moves in steps of a configurable length. From its starting point, it projects a cylinder around itself with a height (both above and below) equivalent to the maximum amount it can climb in that distance. This value is also configurable and using a simple rise over run formula can be obtained as a grade percentage.

This cylinder intersects with the terrain mesh, creating a line on the mesh of where the next point could be. Then a Closest Point component finds the point on that line closest to the goal. This point is fed back into the Anemone loop to continue the pathfinding process until the goal is reached or a certain number of loops occurs. In order to keep the definition lightweight and prevent software crashes, the original terrain input mesh is run through a script that reduces its complexity (Fotiadis 2017).
To improve the script from its initial state, two changes were made within the Anemone loop to facilitate better outputs. Firstly, each new point evaluates the total distance between itself and its end point goal. If this distance is lower than the specified step size, the distance to the end point will be used to specify the radius of the circle for that step. This ensures that the road will eventually meet its end point, as opposed to circling the point due to a constrained step size which occurred in the original University of Pennsylvania script.

The second change was one designed to make the tool produce outputs that might better reflect a real road. Initially, if the tool could not make a straight path over terrain, it would frequently make very tight switchbacks that fit within its maximum gradient criteria. In order to prevent very tight switchbacks which are unlikely to be navigable by vehicles, the script moves away from using extruded circles to using extruded arcs. The internal angle of the arc is definable to allow user control over the tightness of any switchbacks.

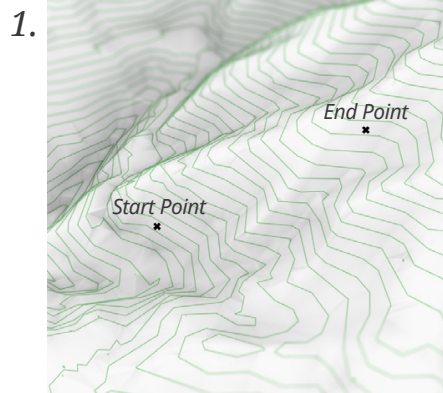

2.
4

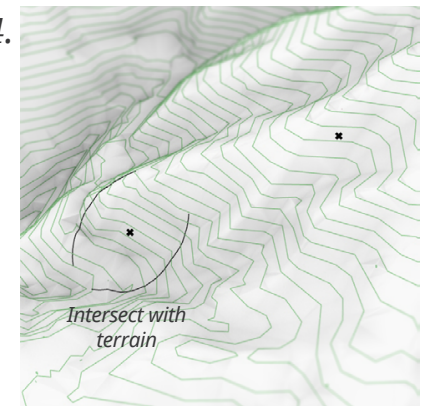

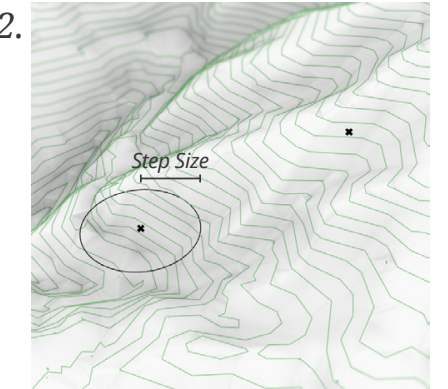

5.

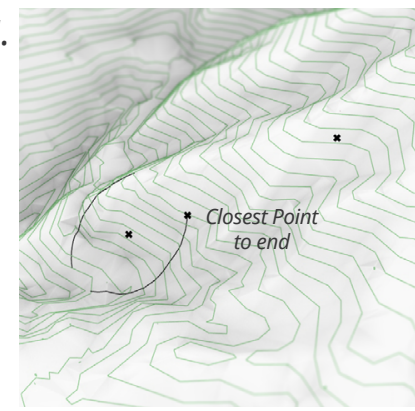

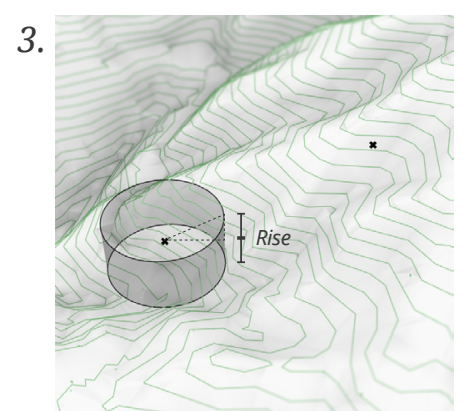

6.
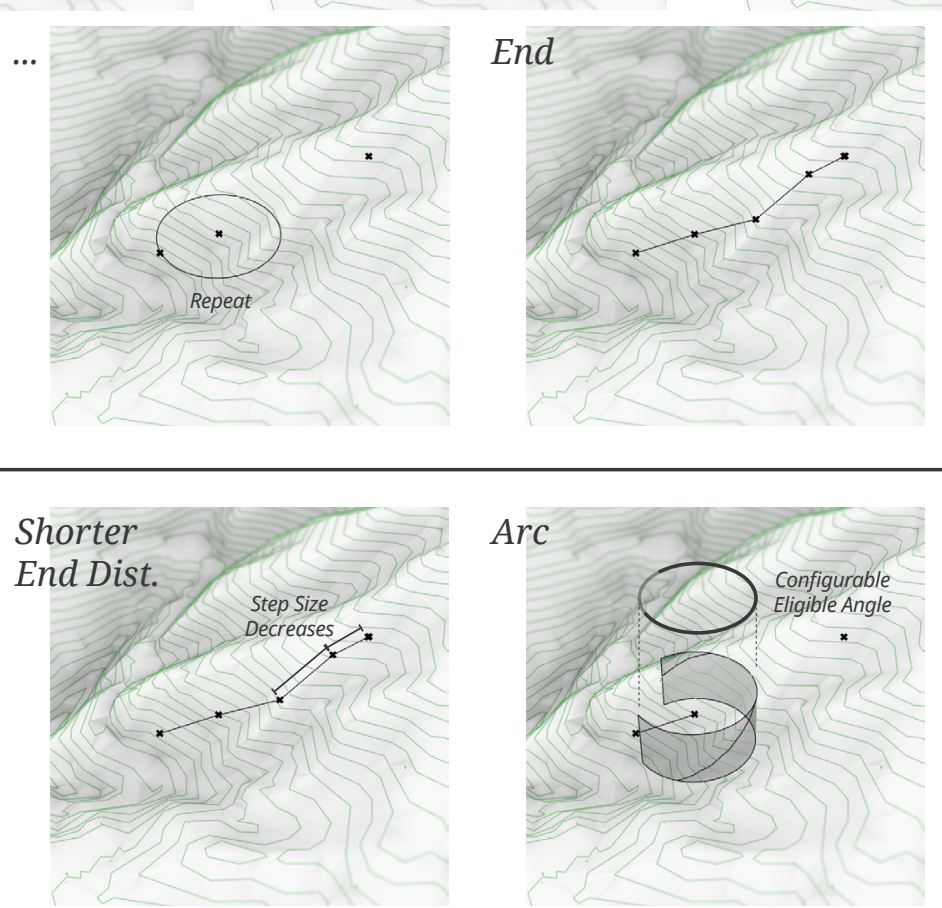


\section{Drainage Model}

This thesis' goal for the tool was to minimise the amount of input required to achieve a result. This included how the boundaries of the site are determined after the mesh is inputted. The pictured example was produced using a drainage direction script (Soler 2012) that calculated how water would run down the surface, highlighting the valleys of the topography as that is where water would collect. By reversing the direction in which the script runs, it can also highlight the ridges.

Unfortunately, this method did not produce a serviceable result and development of the tool moved on with manually inputted calculation boundaries. This experiment was abandoned, though its premise could still be used in future refinement of the tool. The drainage model definitely has promise, and served as a road generator for one of the Computational Planning Group's student semester workshops (Dennemark et al. 2017).

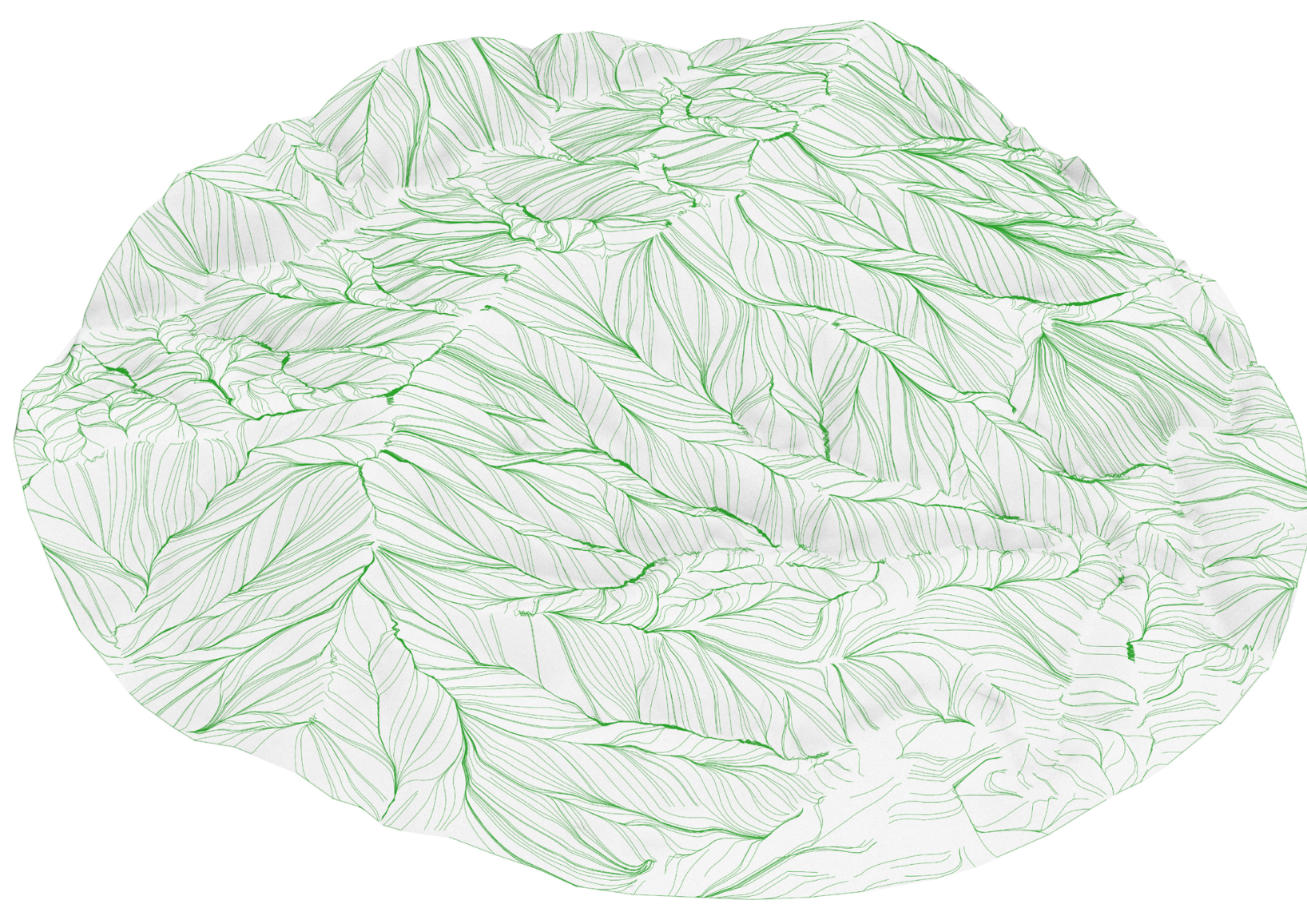

Fig. 5.04. The combined drainage model simulating both with and against the force of gravity, highlighting both ridges and valleys 


\section{Block Maker}

This prototype rationalises the boundaries of a site into a grid system. Lines can be extrapolated from the borders of the terrain model, or drawn on manually and projected on to the terrain.

If the terrain is a valley, a central line must be specified. This line is offset to make a closed shape which is necessary for the block calculations. The spacing of the grid is variable, and two adjustable number sliders control:

- Distance between gridlines in areas where lower boundary is straight

- Distance between gridlines in areas where lower boundary is curved

Where the lower boundary is curved and is convex, a smaller distance may be desired so that roads are not too distantly spaced when they meet the outer edge. Where the boundary is curved and concave, the tool will remove lines that will overlap before meeting the outer edge of the site.

The number of 'rows' (roads that will run perpendicular to the slope of the hill) is variable but applies to the entire system: where the inner and outer boundaries are closer together, the roads will be closer together also.

In the event that the chosen site is a single slope, the top and bottom lines of the desired area simply need to be drawn on the site in the Rhino environment. The script will then generate an urban grid between the two lines.
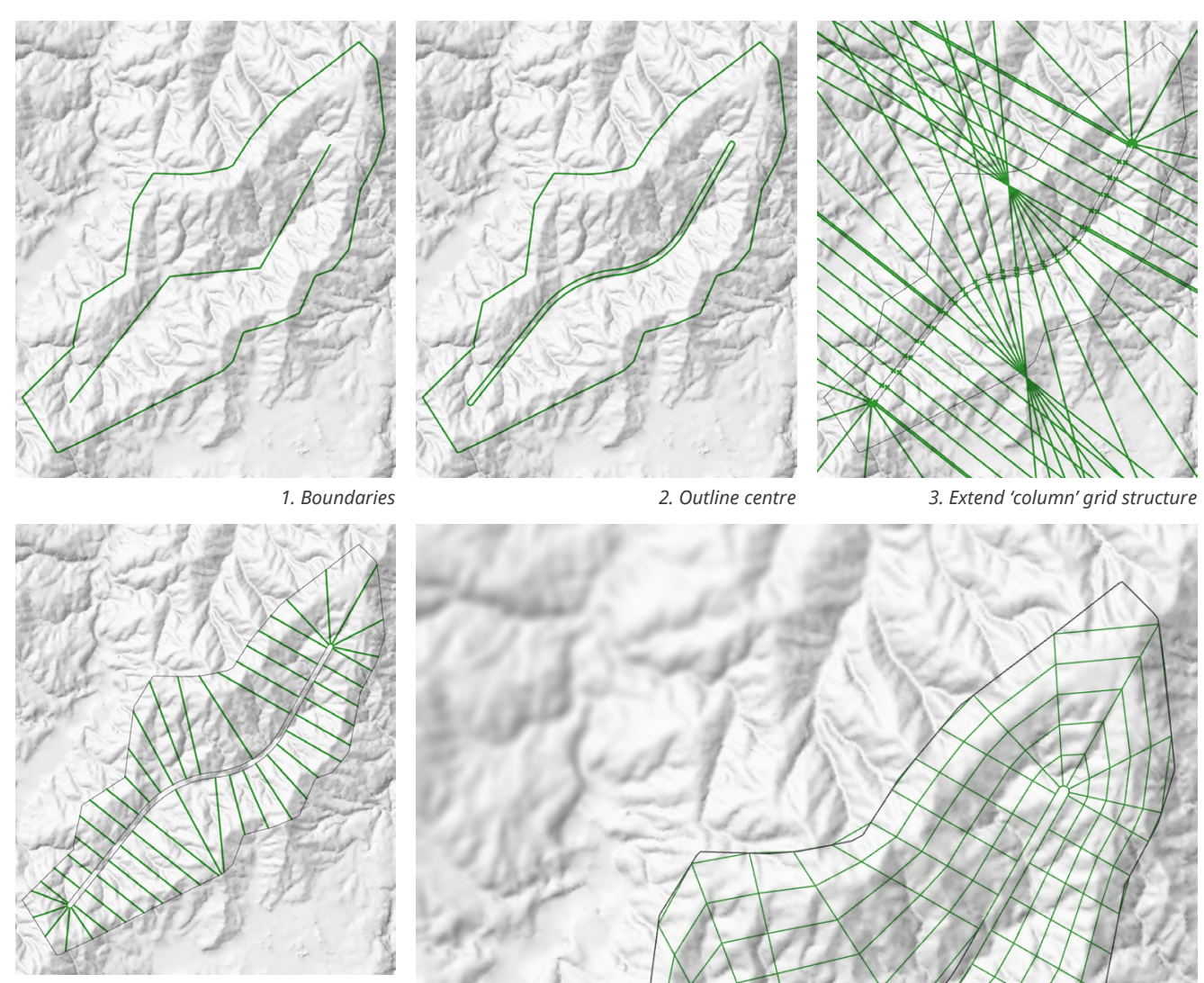

4. Trim and remove overlaps
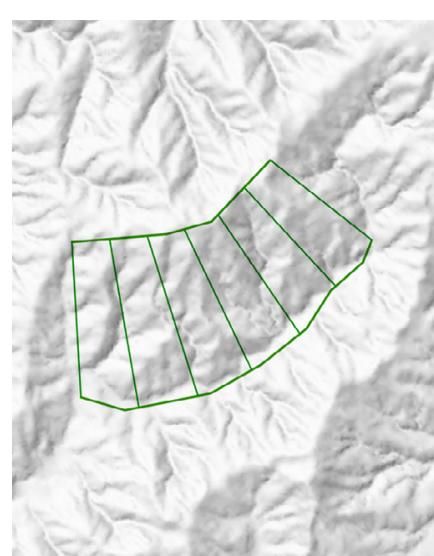

single Slope examp

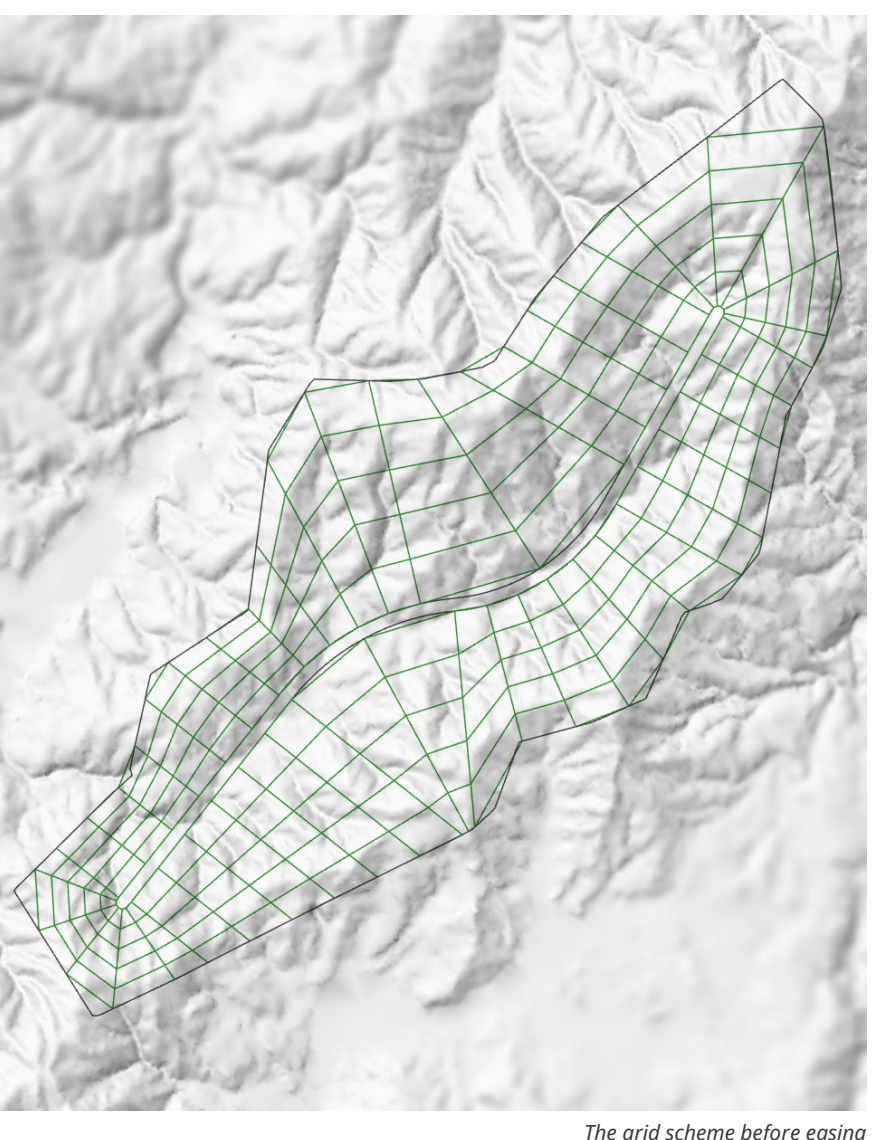




\section{Block Easing}

Taking the grid output from the last prototype, the tool uses the genetic solving algorithm Galapagos (Rutten 2010) to 'ease' the grid and make the connections between each node as shallow as possible. Initially this was done by shifting nodes along the parameter of their host polyline. As the host polylines were those determined by the number of rows, their position was static and this meant that nodes were fixed in one axis of movement. A second version was created which functioned by drawing a circle around each node with a radius that has the circle intersect with the next closest node. Each node is free to move around within its circle, and Galapagos simulates thousands of alternative positions to find the most optimal one for each node within the scheme.

Using a genetic solving algorithm is appropriate in this case as outlined by Rutten is his brief overview of pros and cons of the system in his blog post on Galapagos' release. The algorithms are forgiving in how they operate and so can adapt to tackle any scheme fed to it from earlier inputs in the tool, and are progressive in that 'intermediate answers can be harvested at any time' (Ibid). Full discussion of how genetic solving algorithms work is outside the scope of this thesis, but its ability to test targeted alternatives that are more likely to be successful makes it suitable for this stage of the tool.

The most optimal node positions are based on the following criteria (the fitness function):

- Minimising the slope of the connection with neighbouring nodes

- Minimising the length of connections between neighbouring nodes

How much weighting these factors have is controllable by adding expressions to their components within Grasshopper.

Pictured overleaf is the grid at several stages of the easing process, including image 0 before easing has begun. The genetic solving algorithm starts with drastic changes that are rapidly refined down to find the most appropriate solutions. The 6 images pictured are just samples out of many generations, each generation containing 50 solutions that are 'bred' together to conclude on a refined result.
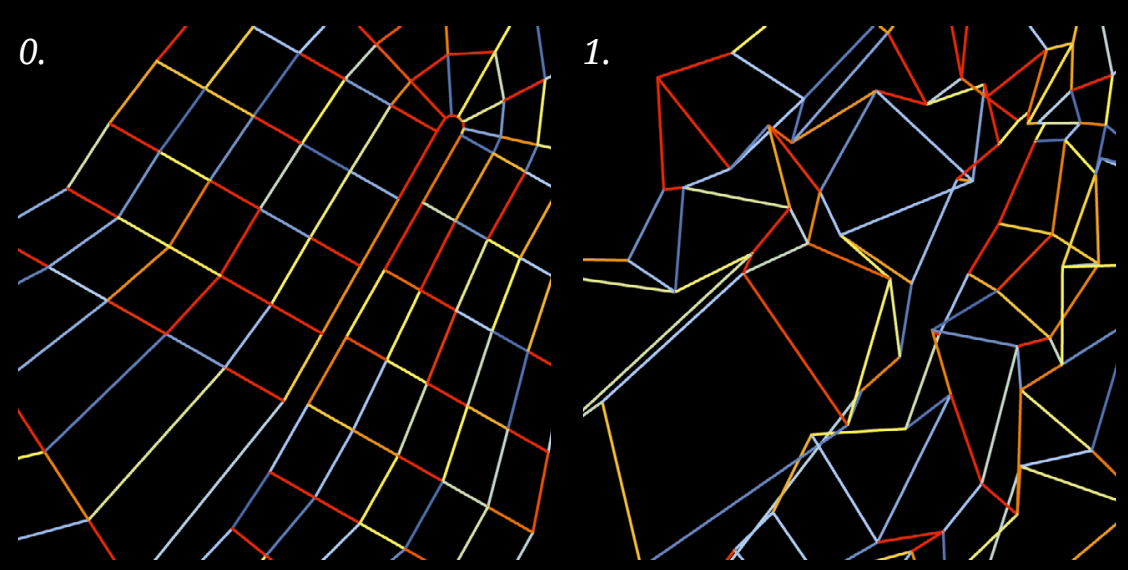

2.

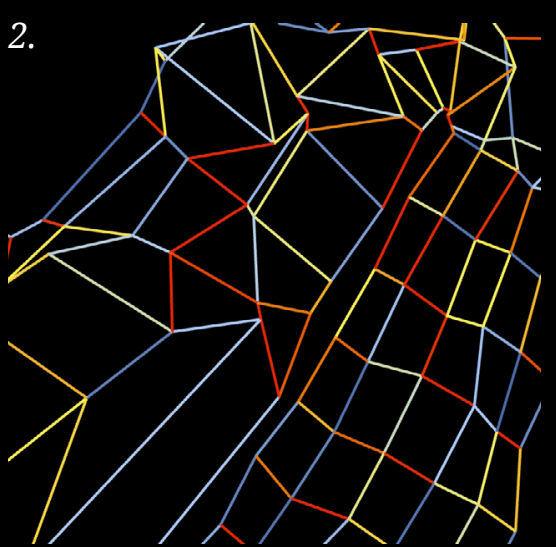

3.

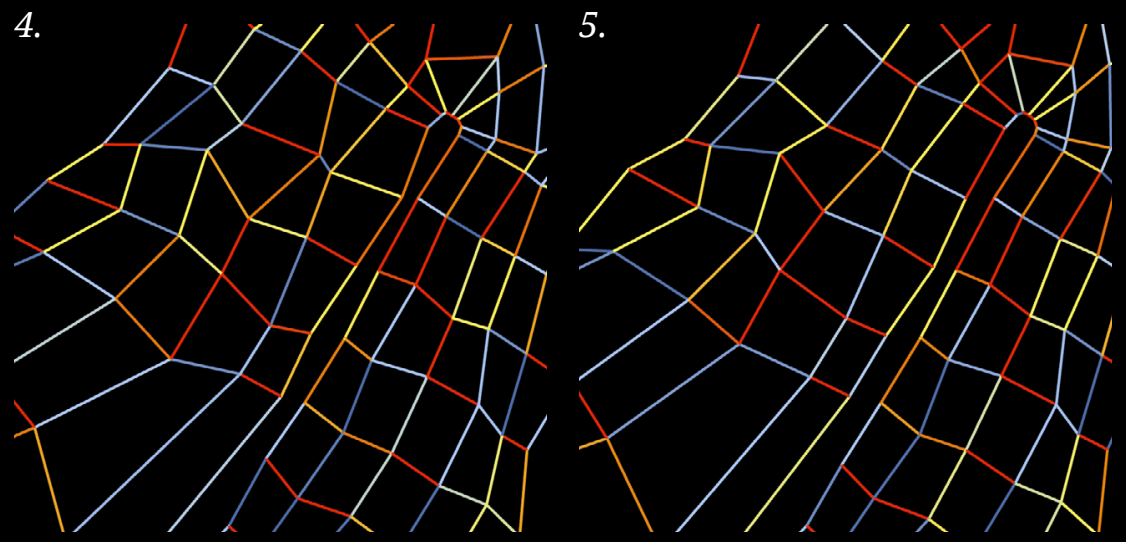




\section{Road Maker}

Though the grid has been eased to have as shallow a gradient as possible, there may be sections that are still too steep to have roads on them. The road maker definition uses the previously mentioned Pathfinding script and applies it to the grid system, dividing the curves of the grid system and denoting a start and end point for each connection. This data structure is then used as the input for the pathfinder definition. The maximum steepness of the pathfinding tool is configurable.

A good default setting for this steepness is hard to find and depends on how extreme the chosen site is. For context:

\begin{tabular}{|l|l|l|}
\hline Source & Street Classification & Maximum Gradient \\
\hline Wellington City Council & Arterial & $5 \%$ \\
\hline & Principal & $6.7 \%$ \\
\hline & Collector or Sub-collector & $10 \%$ \\
\hline & Local & $15 \%$ \\
\hline Christchurch City Council & Public parking access up to 20m & $20 \%$ \\
\hline Steepest Road in Wellington & Private parking access up to 20m & $25 \%$ \\
\hline Steepest Road in New Zealand & $\begin{array}{l}\text { Fore St/Winchester St, } \\
\text { Kaiwharawhara }\end{array}$ & $28.6 \%$ \\
\hline & Baldwin St, Dunedin & $34.9 \%$ \\
\hline
\end{tabular}




\section{Road Evaluation Toolkit}

Due to the roadmaking script being an agent based simulation that works on simple stepby-step logic and on an individual basis, some outputs may not be viable in relation to the network as a whole. These short scripts highlight roads that meet certain criteria, allowing them to be highlighted and subsequently modified or removed.

Total number of switchbacks

Roads are unlikely to be viable if they have too many short switchback segments in close proximity. The script operates by detecting the angle between subsequent segments of the road path, and highlighting roads which have a number of small angles beyond an adjustable threshold.

\section{Overall road steepness}

If a road is at the maximum possible gradient for its entire length, it may be unviable. The script extracts the vector of each path segment and averages the $\mathrm{z}$ component of this vector giving the average gradient of the length of road. It will then highlight roads with an average above an adjustable threshold.

Potential to be replaced by a bridge

If a road passes over a valley (typically a row road), it may be better for the overall network if it is replaced by a bridge. This script averages the height of the start and end point of the road, and then compares it to the average height of each segment of the road. If the average height of the road is considerably lower than the average of its extremes, the road will be highlighted as having potential for a bridge. How much lower the average road length needs to be compared to the extreme points is adjustable.

\section{Proximity to other similar roads}

Some areas of the network, particularly in valley-based systems, may have roads that originate from nodes that are very close together. These nodes would occur in tightly curved segments of the base curve, or where relief has encouraged two parallel roads to travel over the same terrain. This script detects where exclusively parallel roads have come too close together and could be replaced or reduced. They are detected by placing a point on the centre of the road and then creating a circle centred on that point. If a parallel road's centrepoint falls within the circle, the road will be highlighted as potentially too close to its neighbour. The radius of the searching circle is definable to allow for the creation of blocks of all sizes.

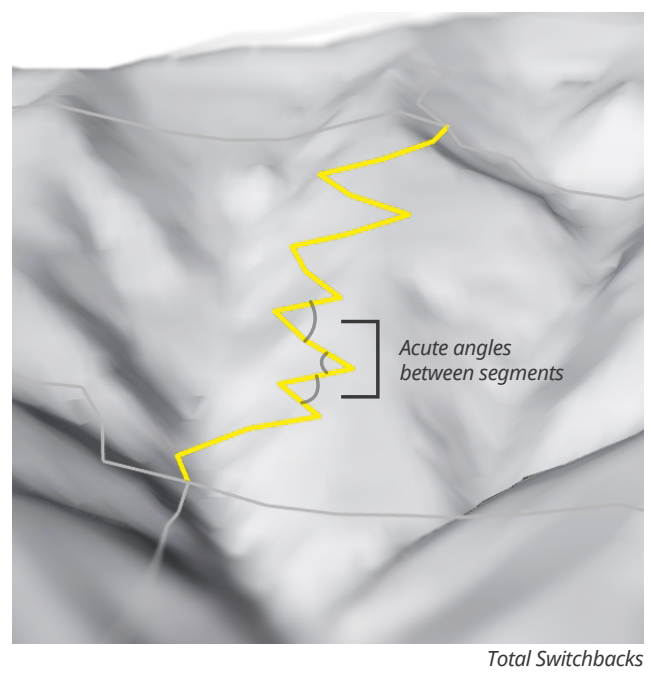

Total Switchbacks

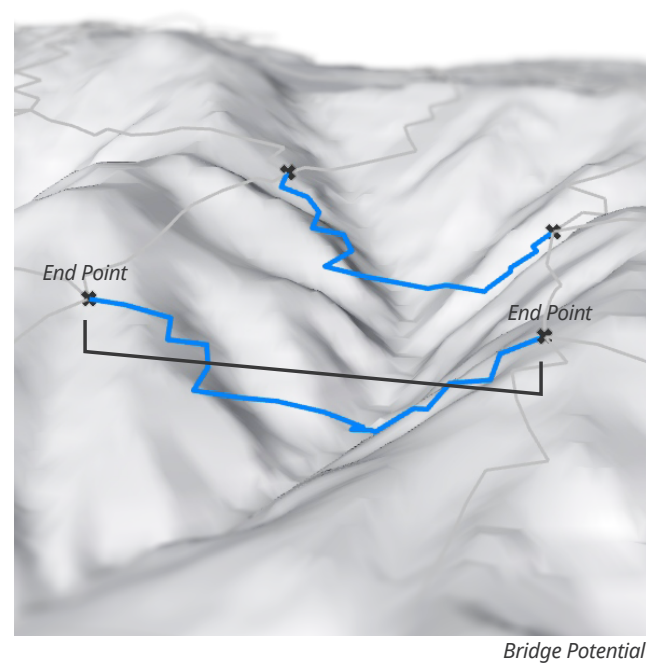

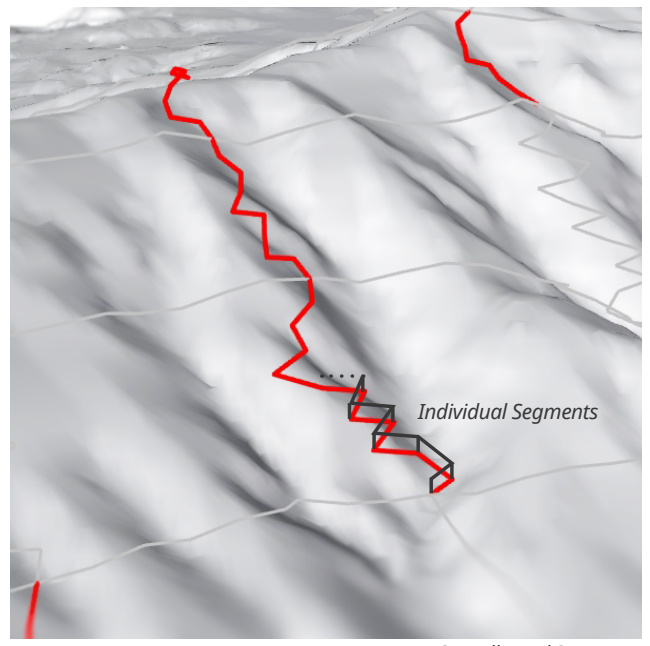

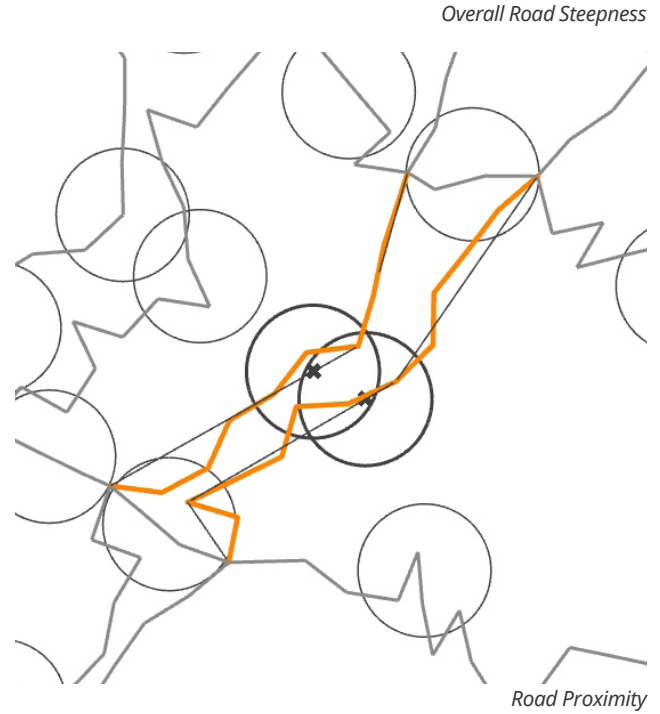




\section{Parcel Maker (Alpha)}

The plugin Heteroptera (Bahrami 2014) is used to extract the topology of the grid network (not to be confused with the topography of the site) and then, using the preserved data structure carried through the Grasshopper definition, is able to apply this topology to the more geometrically complex road network. From here the complexity of the roads is reduced to allow the offset operations to succeed in the most possible instances - leaving the roads at full complexity would cause frequent failures even with a double-pass system included within the tool. By reducing the complexity, the rough geometry of the road is preserved, as compared to operating from the initial block structure which would ignore any curvature in the road.

The resulting simplified roads are offset within the blocks, creating a border of a definable length within the block. This is then divided (again by a definable length) to create packages of land.

In its initial state this parcel maker does not reflect the typical reality of how land parcels are laid out within a city block - certainly in New Zealand there would not usually be empty space in the centre of the block unless it was set aside for a park or public space. There are precedents for this logic in international designs, for example: European high density city blocks frequently have central courtyards within a built perimeter where community areas for residents are kept private. However, dependent on the scale of the overall scheme the tool is being used to process, these courtyard spaces would often be considerably too large and the resultant costs-to-yields ratio would make the proposal very unattractive to developers.

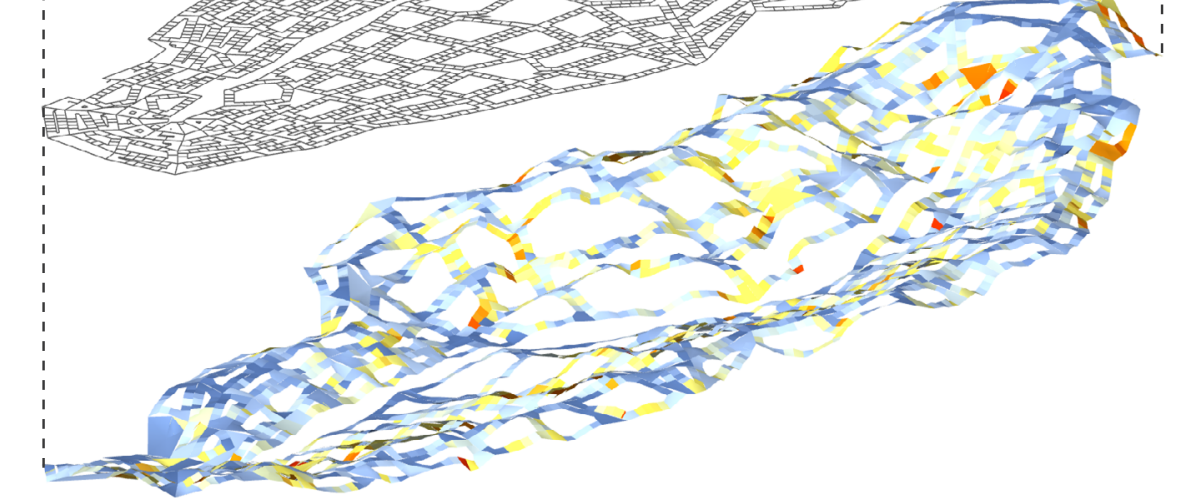

Fig. 5.09. The step-by-step transformations of the parcel maker 


\section{Parcel Maker (Beta)}

In order to generate parcels within a block that utilizes all available land, the Parcels component within plugin DecodingSpacesToolbox has been used. Using Heteroptera to obtain the topology as in the first version of this prototype, the rough polyline boundaries of each block are generated. Again, the road network needs to be slightly simplified to enable the definition to work correctly.

These boundaries are plugged into the parcel component, which outputs a completed parcel solution for each block. The parcel component takes two definable inputs: the width of the street (which is calculated for each block, so needs to be halved), and the minimum length allowed in any parcel. The component will repeatedly divide geometry within the block until it can no longer divide without the output being smaller than the minimum specified length.

These parcels are individual polygons which allows them to have visual analyses performed on them to determine average site steepness, solar exposure, and other criteria that will be important in determining their viability.

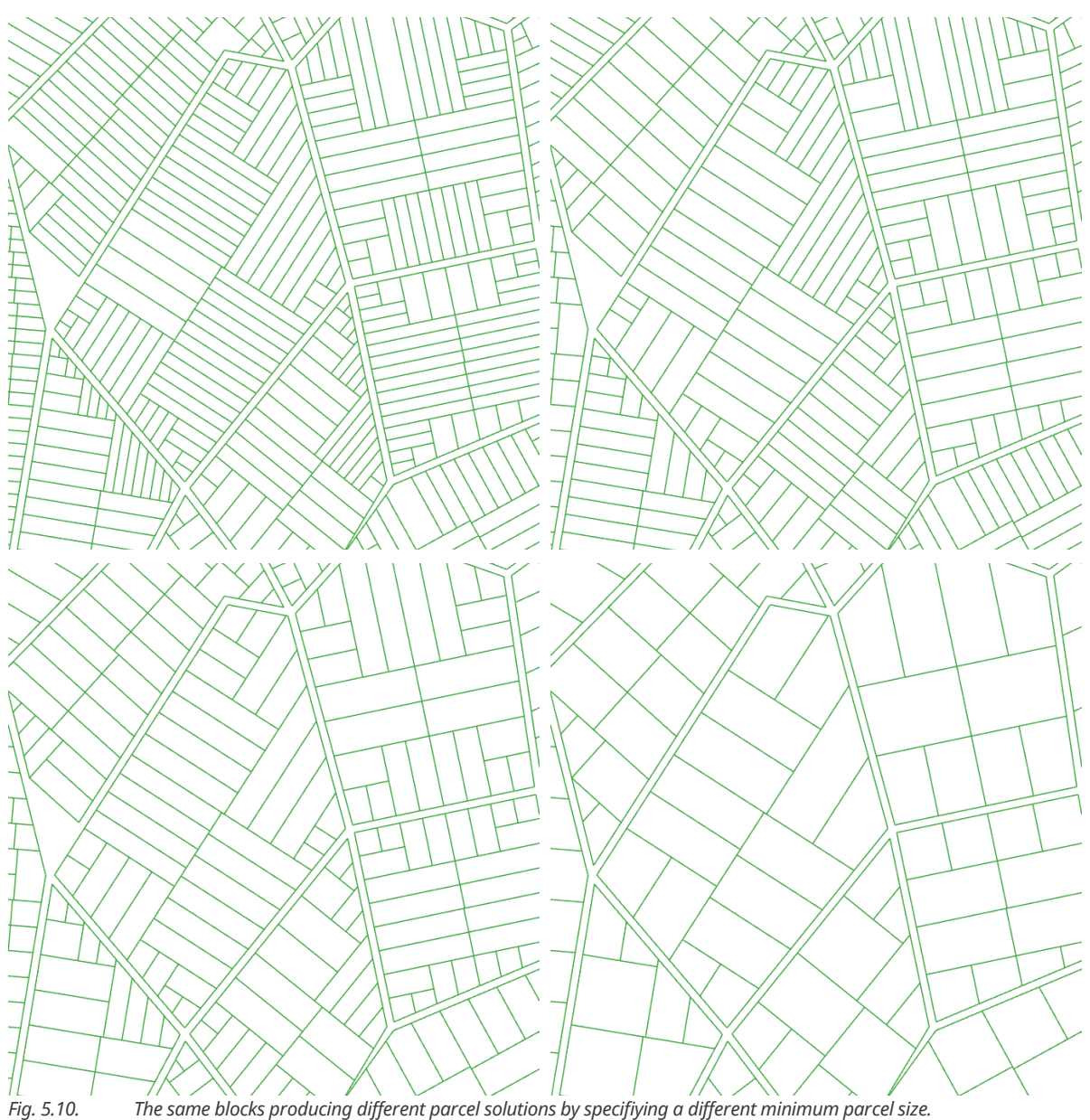




\section{Conerse \\ Proof of Concept: North Makara Valley}

While the previous chapter presented the different scripts designed for the tool and outlined how they work, this chapter provides

a step-by-step of how the scripts link together,

been prepared in a way to make the workflow clear for users unfamiliar with Grasshopper, and those familiar with Grasshopper may benefit from reading it in tandem with the

providing an overview of the workflow from scripts provided in the Appendix of the thesis. 


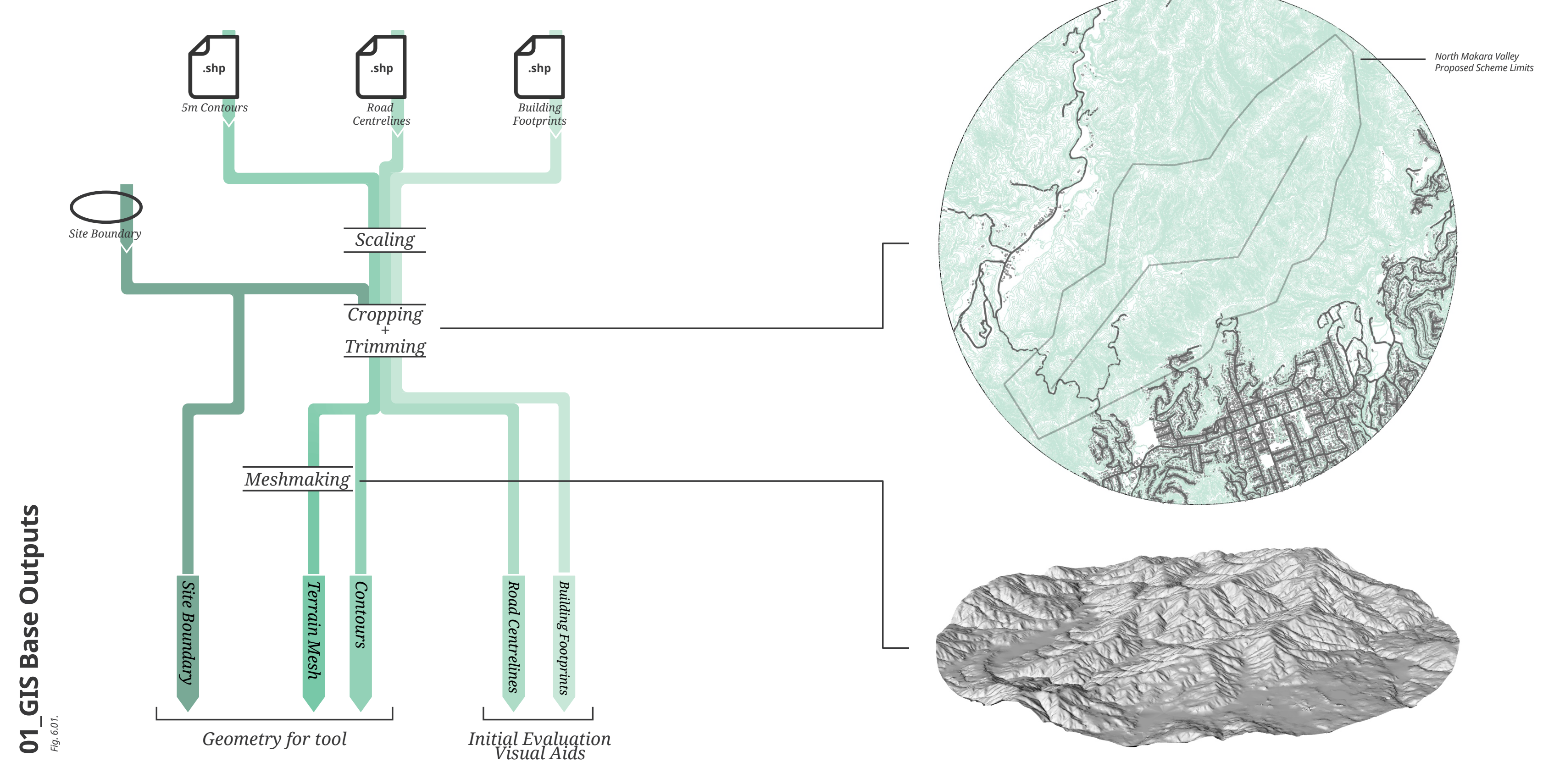




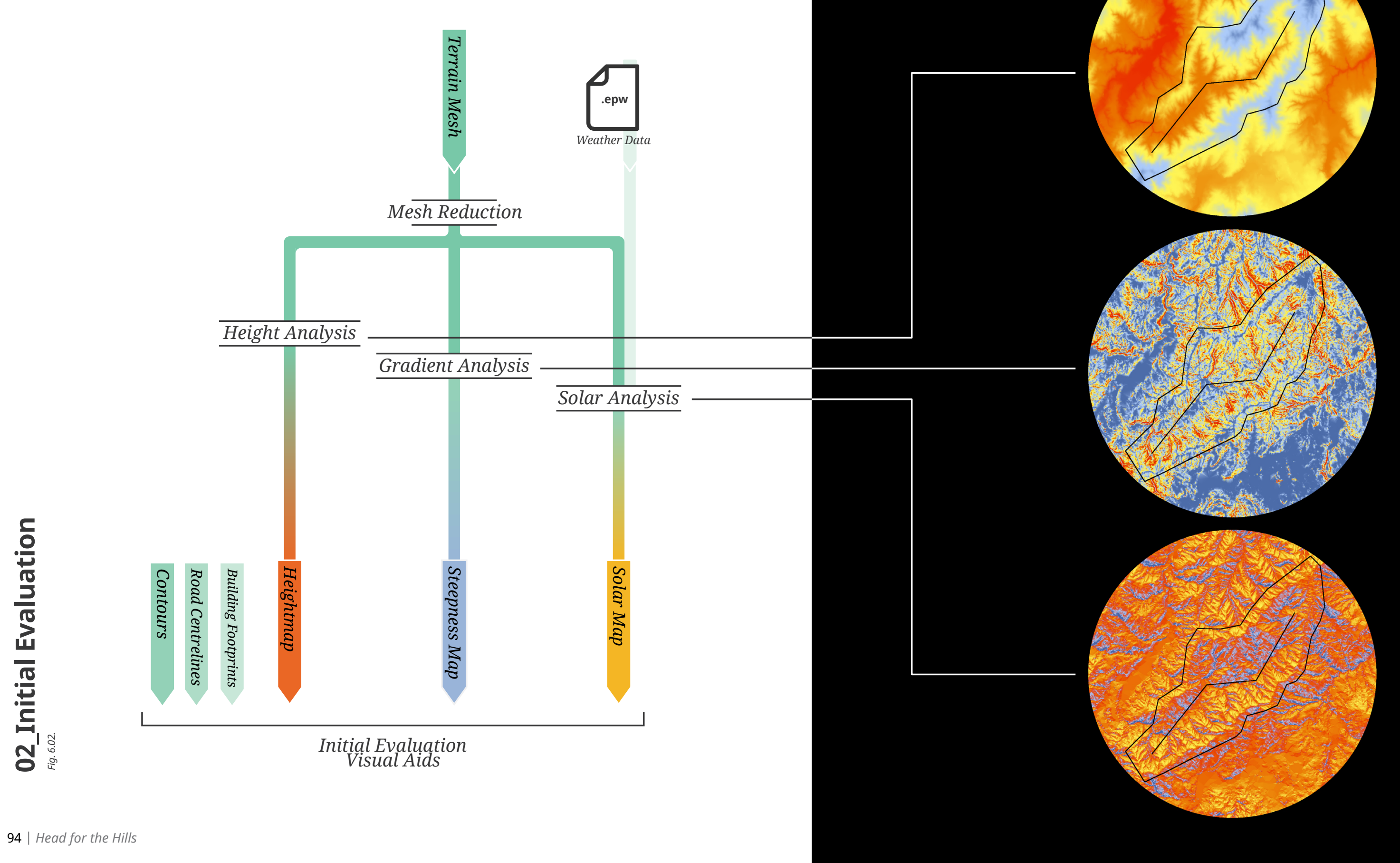




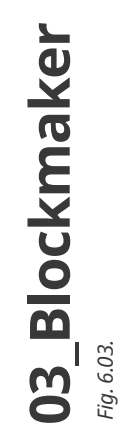
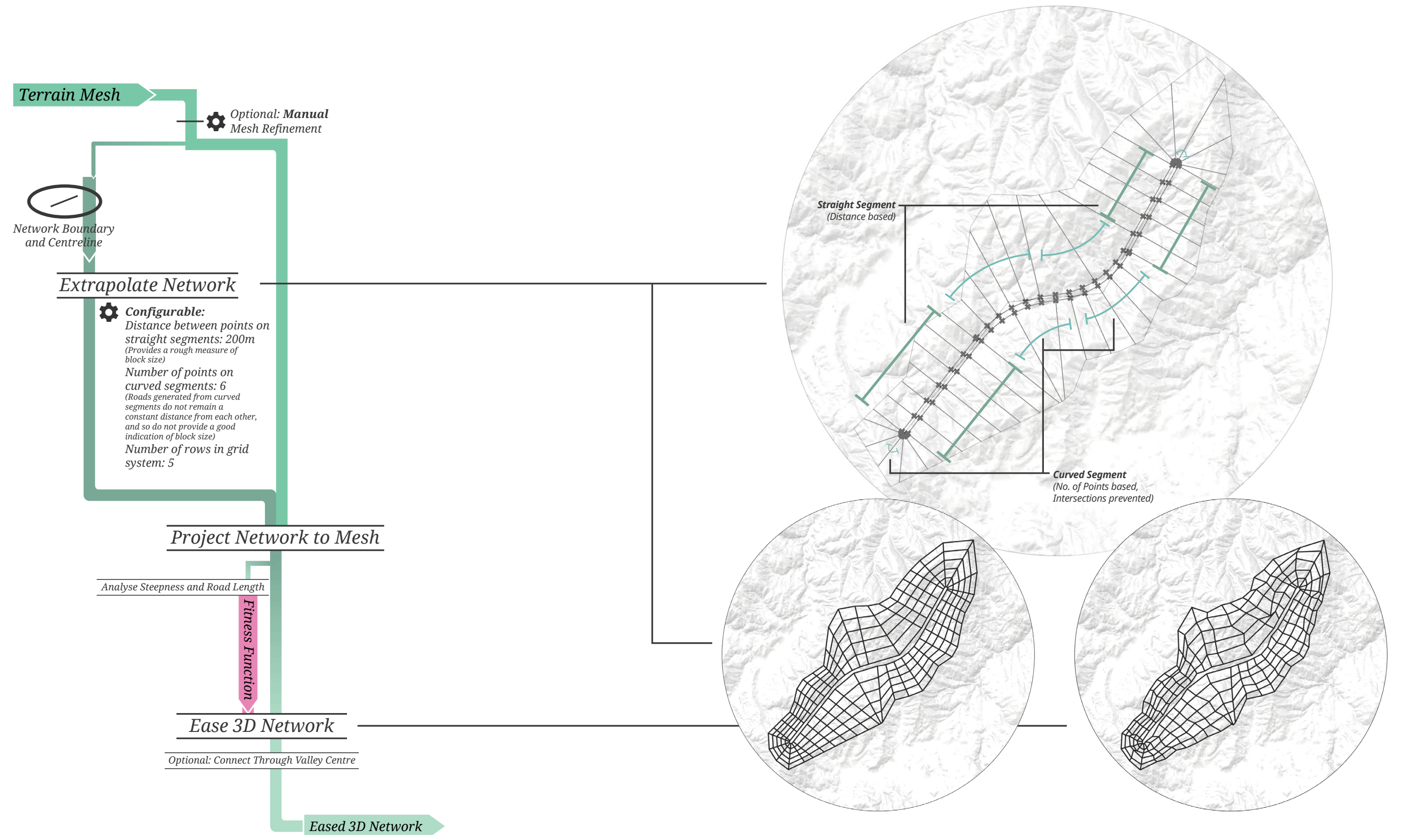


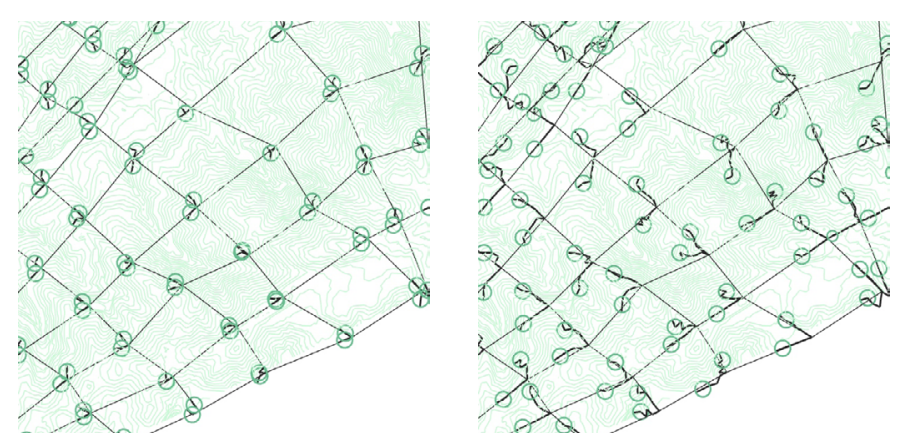

\section{Terrain Mesh}

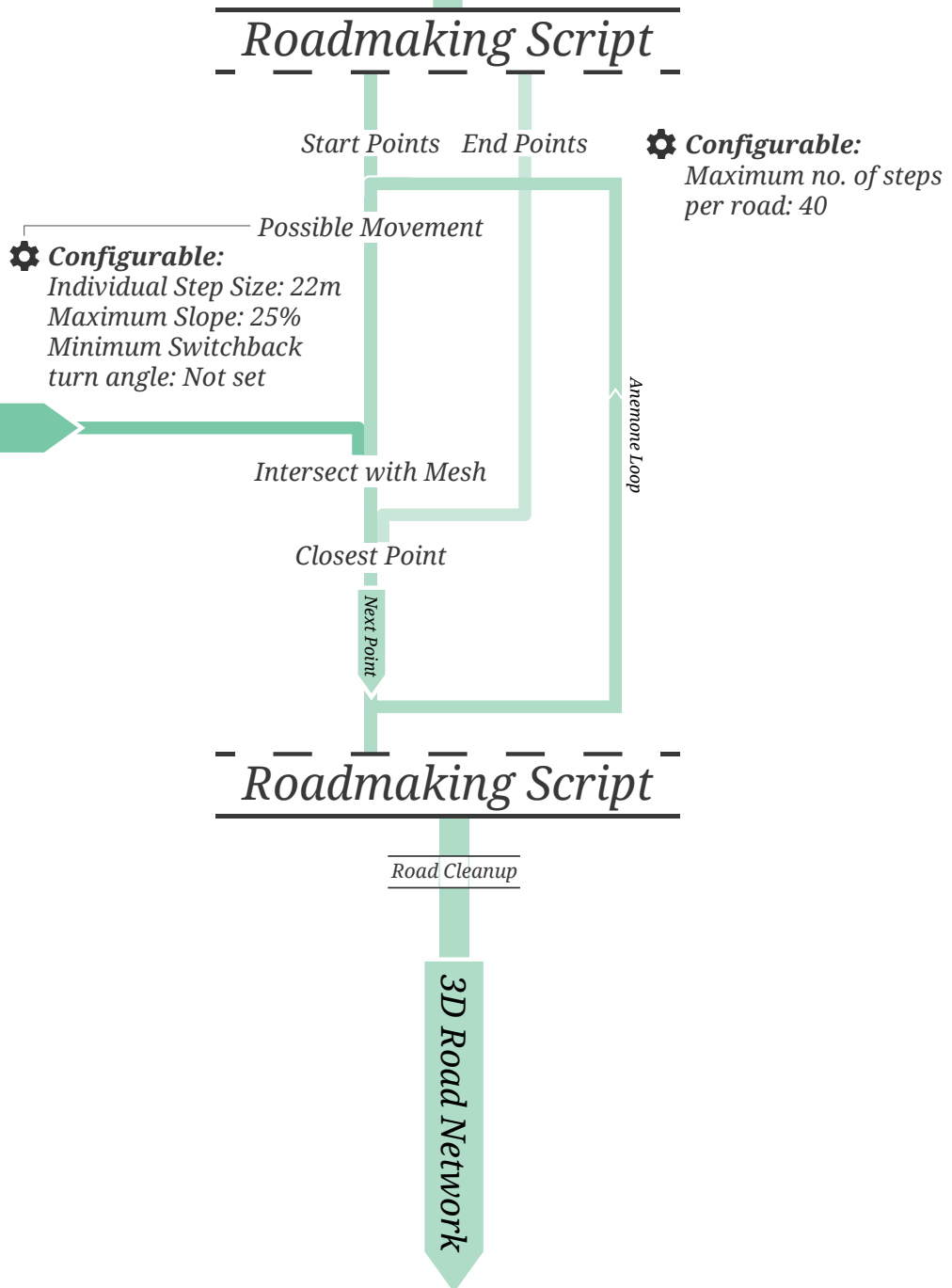

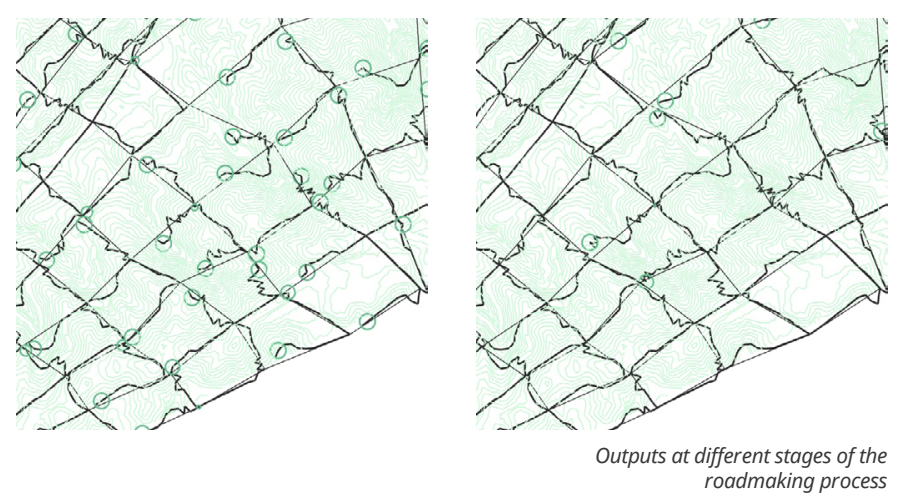

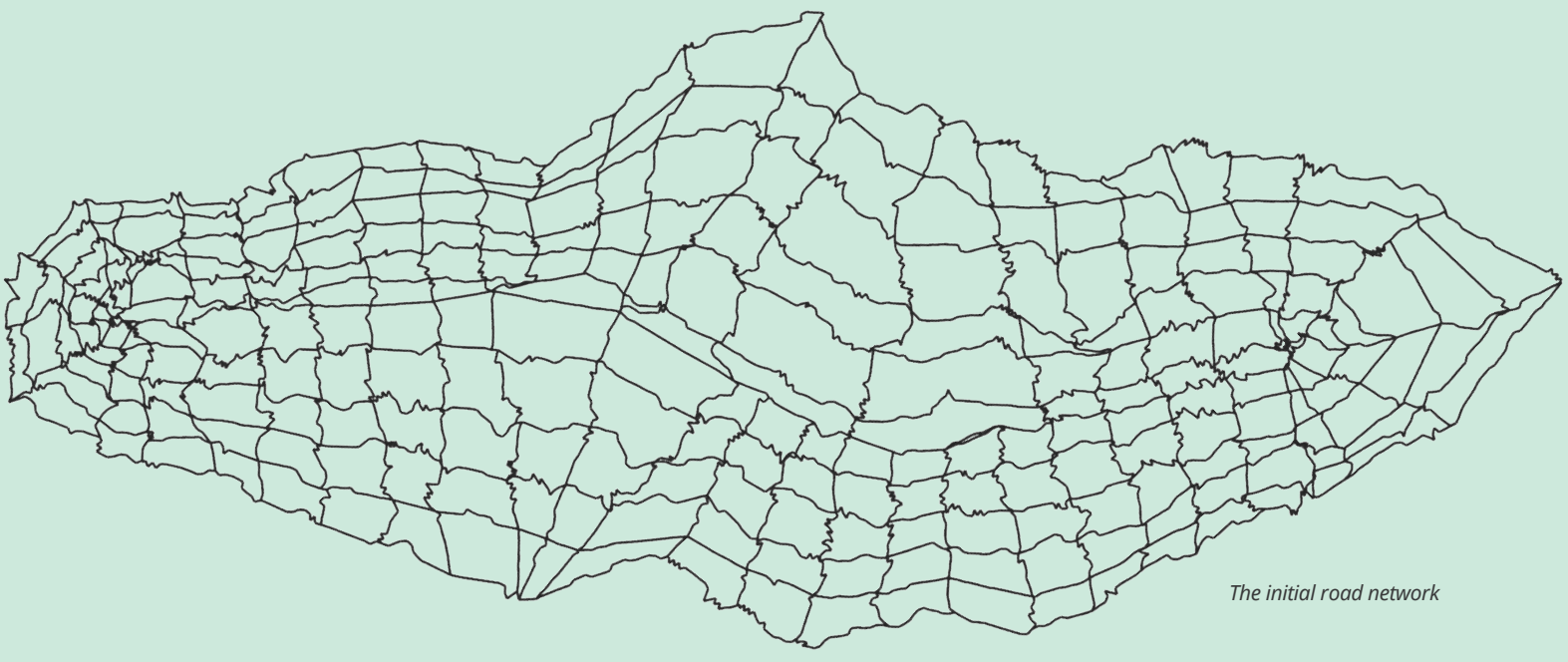



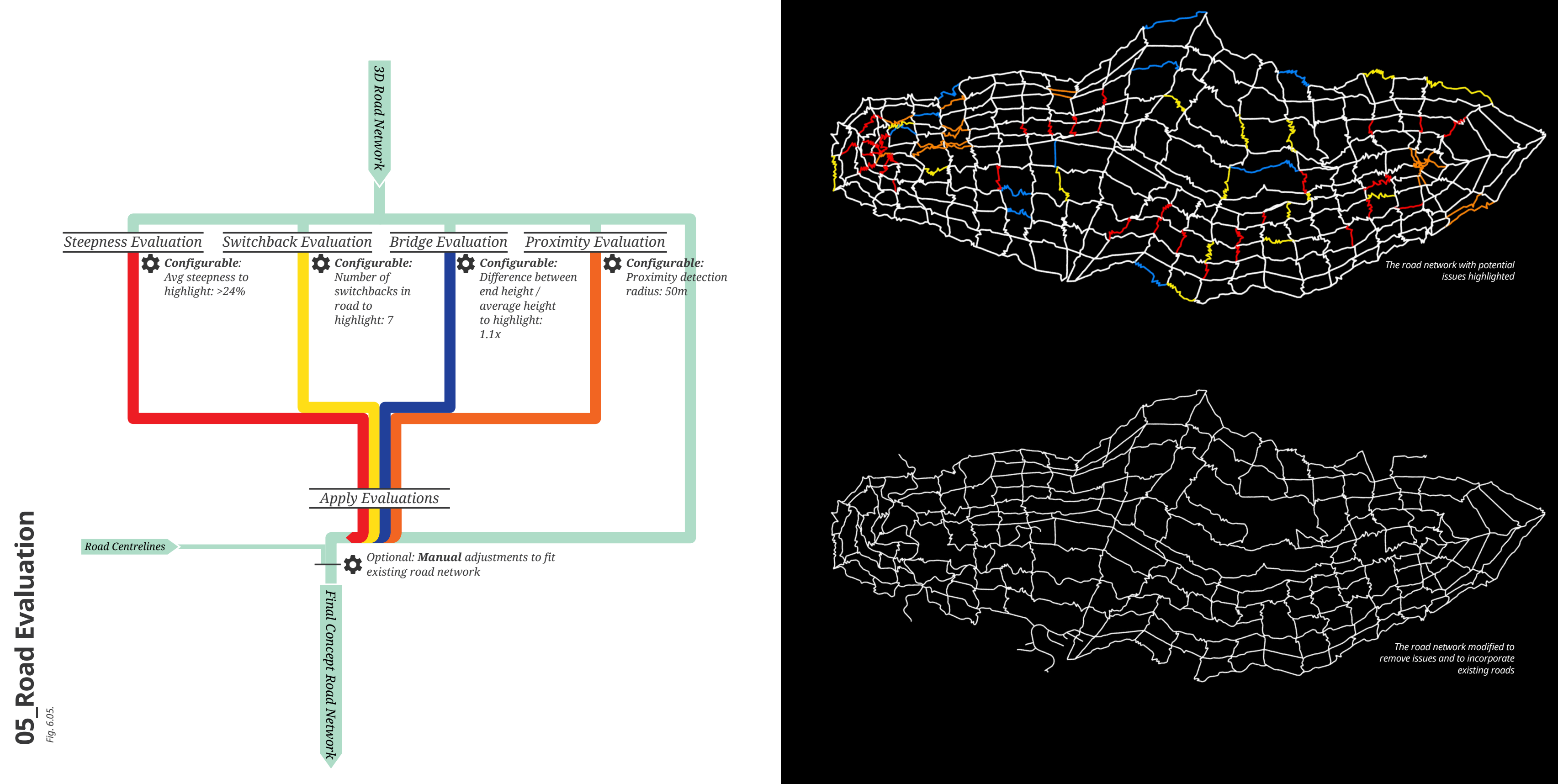

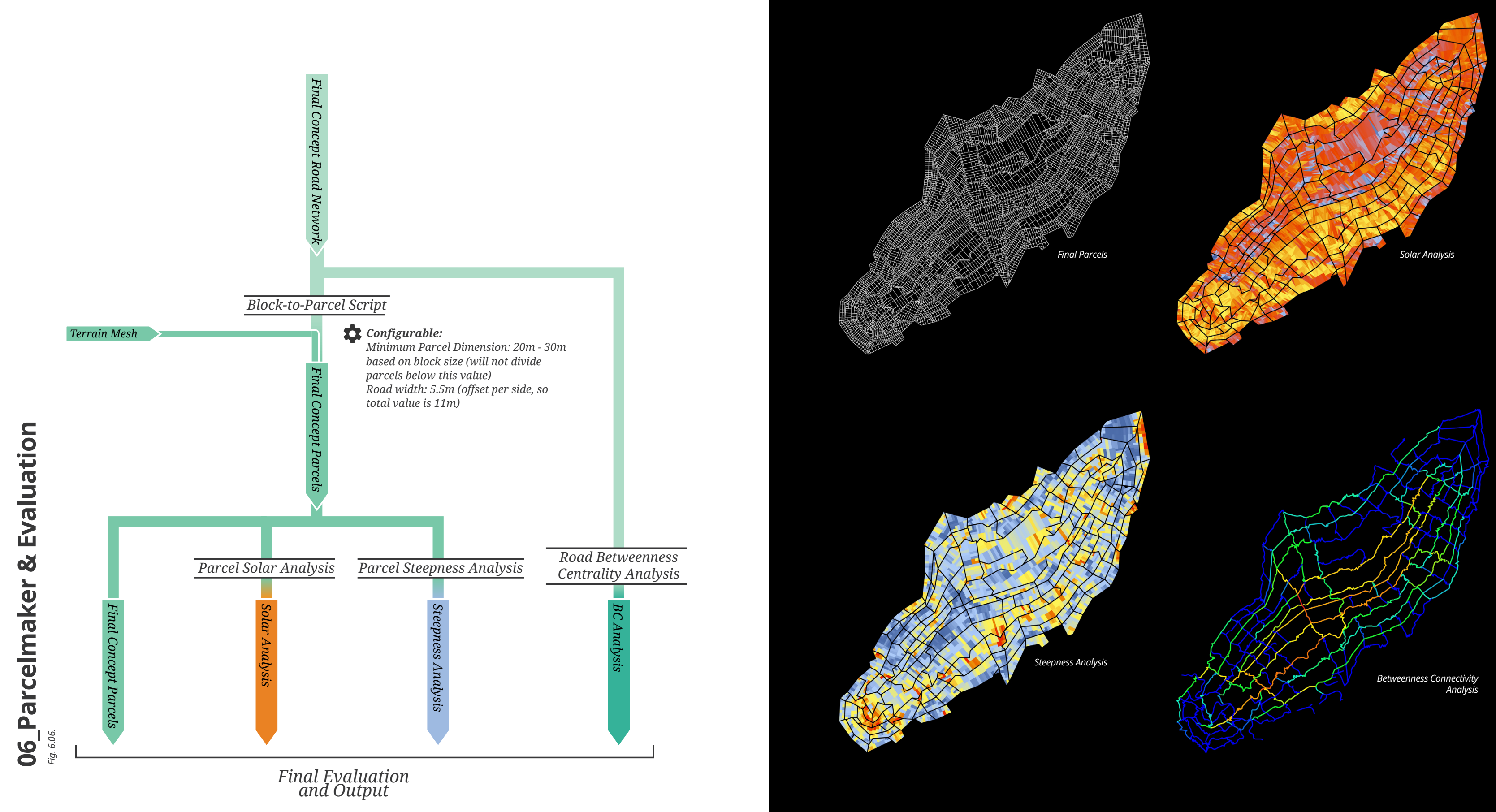


\section{Final Scheme}

After using the analyses provided by the final script in the tool to cull inappropriate sites, the final concept urban scheme for the North Makara Valley is as pictured. The scheme at present contains roads that while winding, do not exceed a gradient of $25 \%$, making them viable for vehicle and pedestrian traffic.

The scheme contains $102.6 \mathrm{~km}$ of road, with $66.1 \mathrm{~km}$ of that road at an angle greater than 15\%. 3826 parcels have been laid out at varying sizes, with the average parcel size being $1529 \mathrm{~m}^{2}$. The scheme covers $5.8 \mathrm{~km}^{2}$, with an additional $0.5 \mathrm{~km}^{2}$ area marked as unviable for building and designated as green space.

For this scheme the areas marked as unsuitable for building were all done so on the basis of gradient: sites were filtered out if their average gradient was higher than $15 \%$. It could be argued that sites on steeper gradients could still be viable, but this value has been chosen for the sake of demonstrating the ability of the tool to modify its outputs to fit chosen criteria.

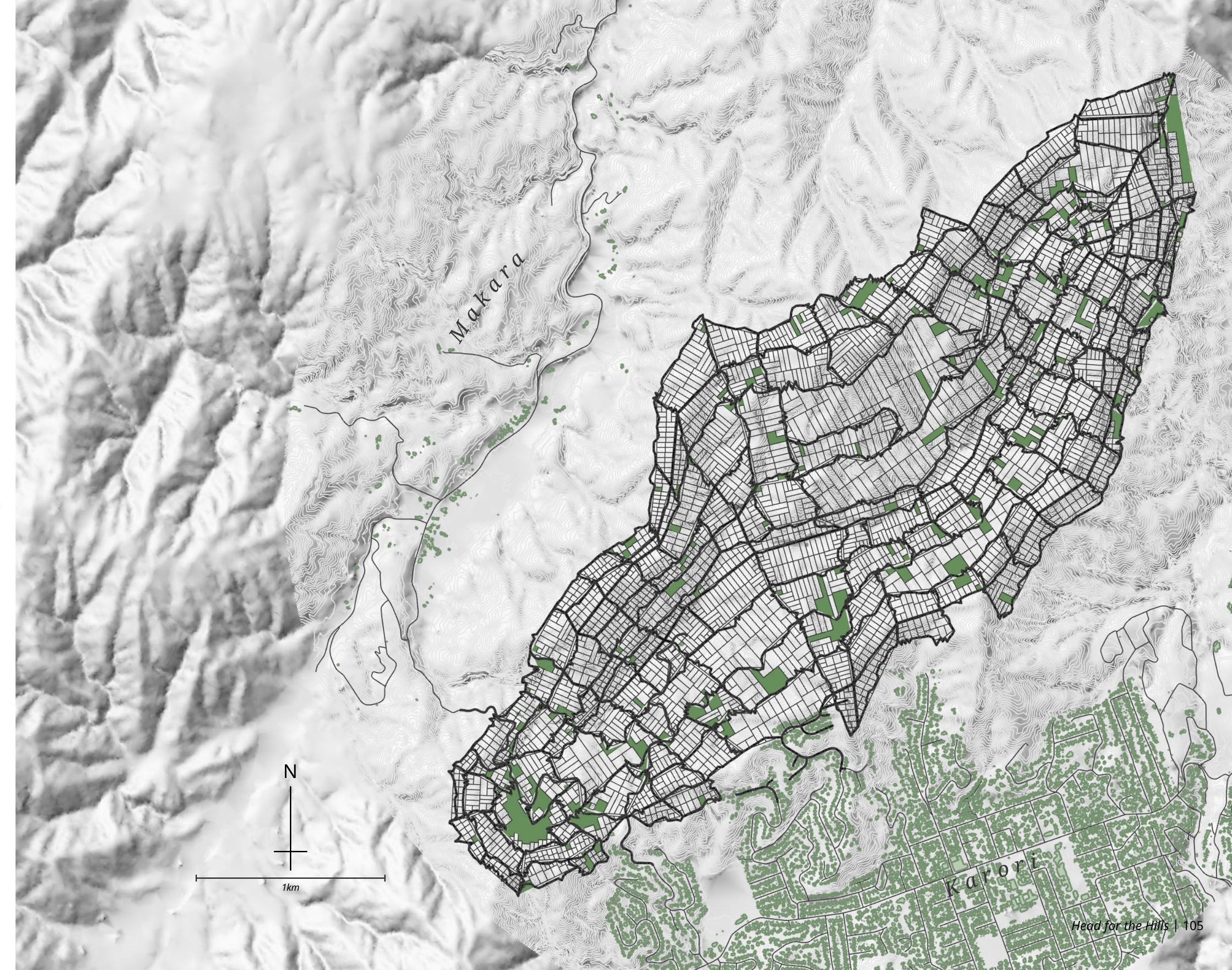




\section{Data Structure}

Grasshopper handles information in three different ways as it passes from component to component: a single item, a list of items, or a tree. A tree in layman's terms is a collection of lists assigned to 'branches' that keep them separate, and when two trees of data are used as inputs for a single component, the component will run several times using the data on matching branches.

As the information passes through the tool from GIS information to the final scheme, it undergoes several changes in structure: 'grafts' (moving information into more complicated branches) or 'flattens' (removing branches and having all data in a single list). Care has been taken to ensure the data structure throughout the tool will have common features no matter the site in use, enabling the retrieval of specific data over the course of the tool.

When data in the tool is flattened, much of this relationship information is lost. The most important instance of this is when the road network is flattened when 'baked' over to Rhino (made editable in the Rhino environment). It is possible to restore the data structure by comparing the modified road network with an older version with tree structure intact - the script occasionally does this by giving each road a unique signifier which is the addition of its start and end points coordinates, a value which remains constant over that later part of the tool. This does not work, however, with roads that were not initially part of the network, such as existing roads the tool user may have incorporated into the network through manual modification in Rhino at the evaluation stage.

As fig 6.08 shows, the tree structure that is constant across all possible sites is that of the road network from when it is generated to when it is evaluated. The roads are split into 3 branches at the base of the tree: 'column' roads (those perpendicular to the contour lines), 'row' roads (those parallel to the contour lines), and the optional third category of roads that bridge between the empty centre of the valley that is created when the base curve is offset. Column roads are always at address $[0, \mathrm{x}, \mathrm{y}]$ within the road network data structure, where $\mathrm{x}$ is the number of the road from centre to boundary, and $y$ is the number of the individual segment of road between nodes. Similarly, row roads are always at $[1, \mathrm{x}, \mathrm{y}]$ and valley connector roads are at $[2, \mathrm{x}]$. This consistency in data structure enables any future modifications to the tool to target specific roads within the network.

\footnotetext{
Site Mesh Site Mesh

Valley Centreline/ Boundary

Grid System Trees - Column Road (0,x) Row Road (1,x)
Valley Connector Road $(2, x)$ Road System Trees - Column Road (O,x) Row Road $(1, x)$
Valley Connector Road (2,x)

Modified Road Scheme Flattened List

Blocks and Parcels $\bigcirc$ List $\bigcirc$ Tree Fig. 6.08.
}

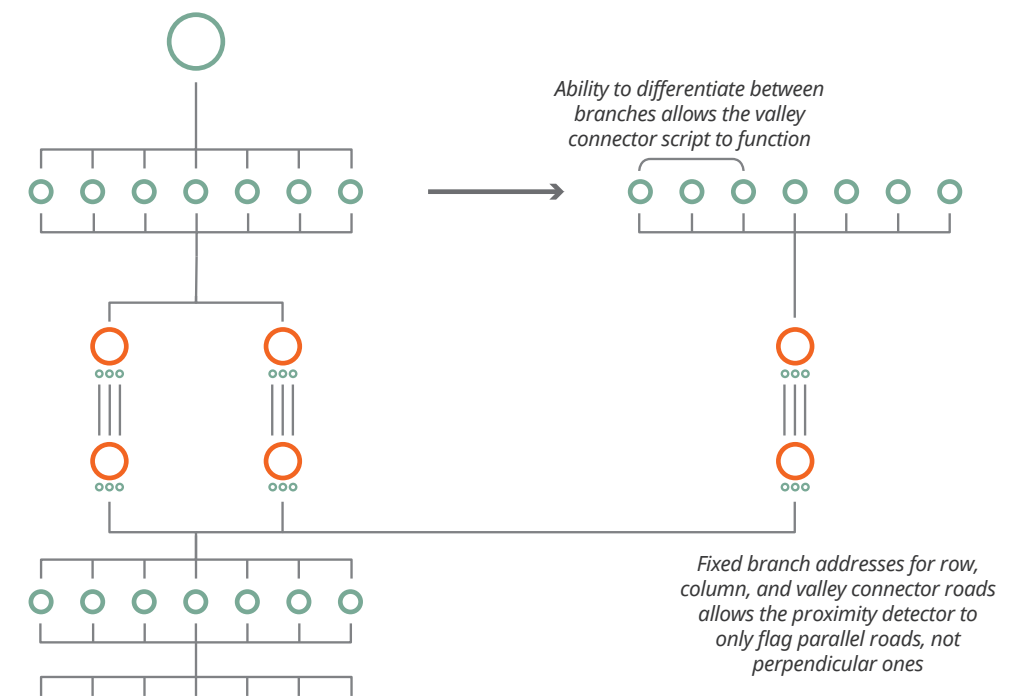

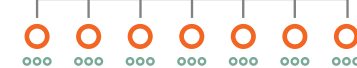

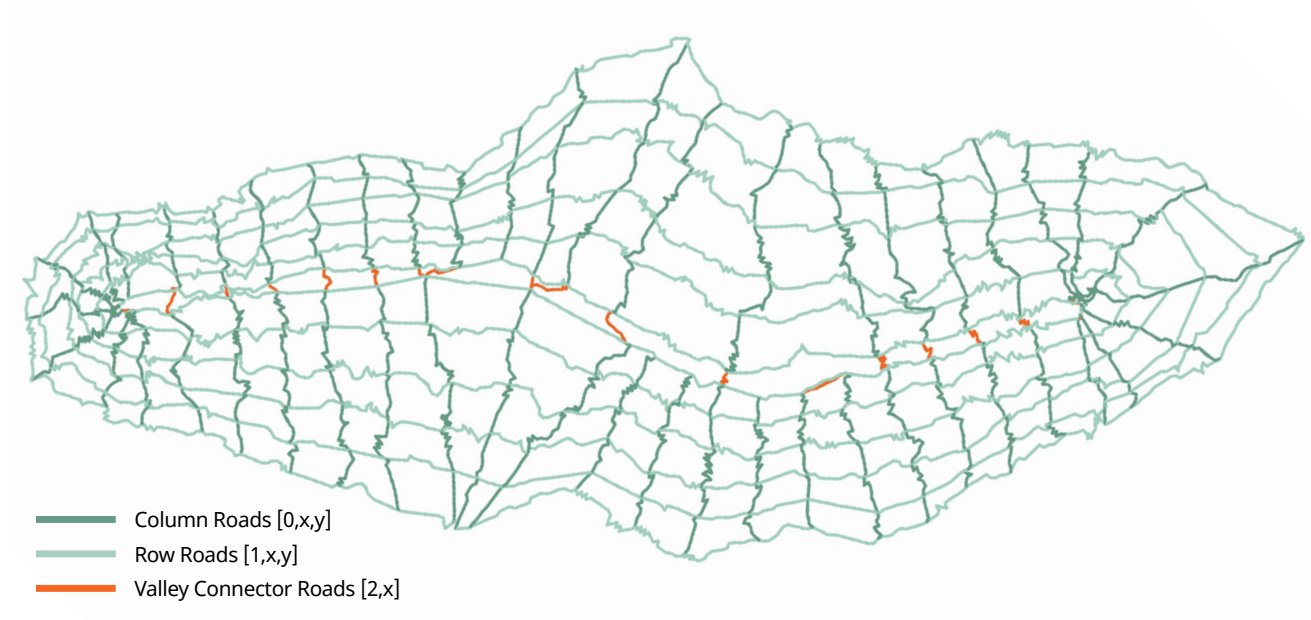

Fig. 609. 


\section{The Tooling Process}

The previous two chapters compiled the bulk of the work done over the course of this research: development time on scripts, experiments with how best to assign a logic to a code, and geometry generation concepts applied to different parts of the process. The resultant series of scripts, including the final overarching workflow and its output, serve to demonstrate how effective Grasshopper is as an environment to construct tools.

Grasshopper's strength comes in its modularity: every part of its process could be considered a module, from a singular component to script that serves as a link in the chain of an overall definition. Urban plans also suit being designed in a modular, step by step way given their scale and complexity. This was a central premise of Towards a modular design strateg for urban masterplanning (Dennemark et al.

2017), a paper which recorded the output of several student groups developing their own generative design tools in modules. Each module from each student group could easily be swapped out for the equivalent from another group. That paper, Head for the Hills, the Grasshopper-based pattern languages of Beirao and Duarte - all exist within the same environment, and thus elements from each could work together in sequence with some simple adjustments.

This creates an environment of open source collaboration, as can clearly by seen by the wealth of plugins and extensions that have been applied to Head for the Hills. Within this network of collaboration, there is a great potential for tools, whether entire scripts or just modules, to be developed for a variety of unique situations. In the context of this thesis it could mean one set of scripts for a certain terrain typology and another set for different terrain - the potential for additional research is immense.

Within the frame of the research set out in the methodology, the creation of the tool has been a success. The tool is capable of outputting a conceptual scheme using only map data and designer guidance. The usefulness of the scheme as a concept will be reviewed in the following chapter. 


\section{conetr Evaluation}

This chapter seeks to evaluate both the success

of the North Makara Valley scheme as a

valid piece of urban design, and the tool that generated it as a designer of urban design.

This evaluation is done using the established

with existing Wellington suburbs. Discussion of the tool's efficacy is aided by more direct comparisons of its functionality with existing

evaluative framework schemes by using rapid concepts generated

combined with a qualitative comparison 


\section{North Makara Valley Evaluation}

As the proof-of-concept scheme of Head for the Hills, North Makara Valley shows that the tool has merit in generating urban schemes.

Examining the Betweenness Centrality analysis, the scheme has its brightest bands occurring in the 'row roads', which run parallel to contour lines and thus are the least steep. Concentrating traffic in this band makes the most sense as the shallower gradient and fewer switchbacks will make for smoother circulation. In a real life scenario, these roads would be specifically built to be major circulation routes and would be wider than other roads in the scheme, as well as having more earthworks undertaken to smooth over terrain where possible.

By nature of being in a valley, segments of the scheme are quite flat. This is important in the context of the overall urban plan, as it provides a location for buildings with land uses that cannot exist on steeper sites. It also provides a place for people with impaired mobility to live and move without terrain obstructions.

There are larger blocks in the scheme that correspond with south-facing areas of the valley that receive less sunlight. Concentrating areas where people live in terrain with more solar exposure makes sense from an energyusage and sustainability perspective, so it is good to have the larger parcels that may see alternate uses such as pasturage in areas that receive less sunlight.

Allocated throughout the scheme are areas of green space, which would be able to serve as public areas. While not able to host the playing fields one might imagine in a typical park, green areas on steep terrain are still fit for purpose with quality design intervention. The bulk of Wellington's parks and reserves are located in difficult terrain, with ample walking trails and other amenities still available despite terrain complications. Steep terrain actually offers additional design opportunities for children's playgrounds, as seen below:

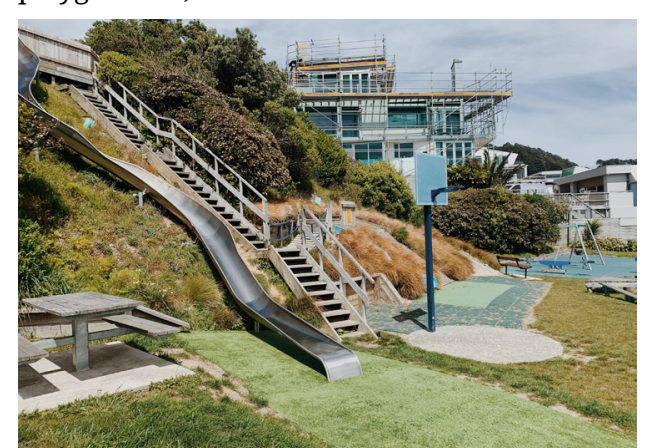
Fiq. 7.01. Roseneath Park

Further development of this scheme would likely see expansion on the amount of green spaces: the ones presented here are simply the ones that can make best use of otherwise unsuitable sites. Depending on the intentions of the tool's users, how much space is reserved for public spaces would come down to whether the developer wanted to maximise yield or improve quality of life for inhabitants.

The scheme also provides good insight into
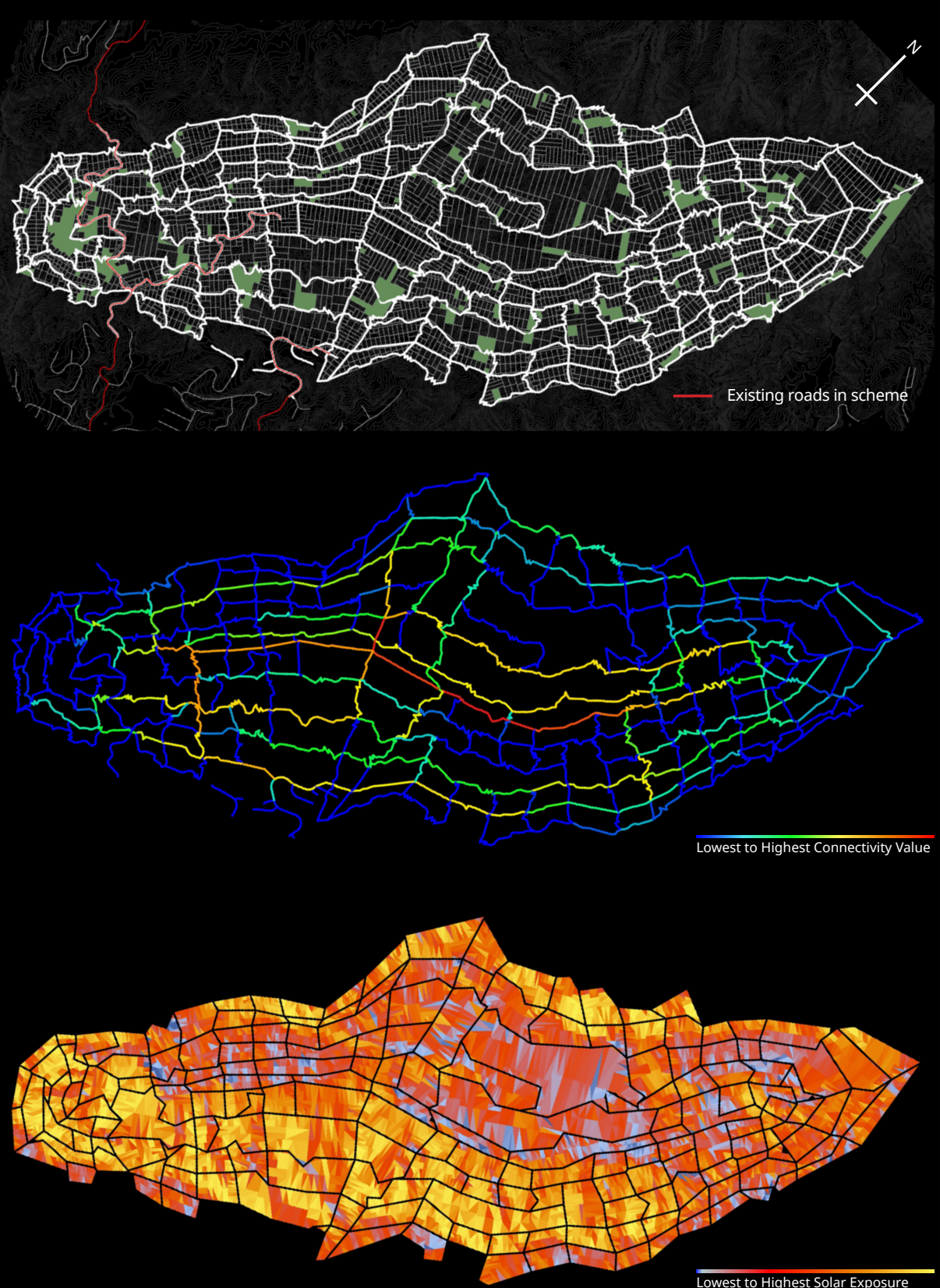
how well the tool is interpreting the site. A good metric of this is how well it begins to suggest roads that align with existing roads. These existing roads have had earthworks done to build them, and are reflected in the contours of the site. As a result, the tool should interpret this as a good area to place a road. In this scheme the tool successfully aligned a road with Makara Rd, which runs from Karori to Makara Valley through the south end of the site. This made it very easy to incorporate the road into the overall scheme. The scheme also connects to the top of a Karori network of culde-sacs along the skyline walkway. A practical scheme would likely have a third way out of the valley, probably at the north end travelling to Wilton.

This scheme used an easy-to-implement twovalue system to feed its minimum block width to the parcelmaker component. Measuring the overall area of each block, the parcelmaker was then fed a number based on whether the block was over a certain size threshold. Larger blocks had a larger minimum size and therefore larger land parcels. This sort of utility is the beginning of what could be a zoning process built into the tool's logic, with areas with less roads holding lifestyle blocks and considered more 'rural'.

Because of the overall size of the scheme, the tool was limited in how small a minimum parcel size it could specify before the script would start crashing. On a smaller scale the tool would be able to specify narrower parcels to improve density in parts of the scheme.
Overall, the scheme is a successful one, particularly considering the chosen frame of evaluation that is Space Syntax-based network connectivity and similarity to preferential qualities in existing Wellington suburbs. Its success, along with the factors mentioned previously, suggest the tool is attaining its goal of generating valid urban schemes.

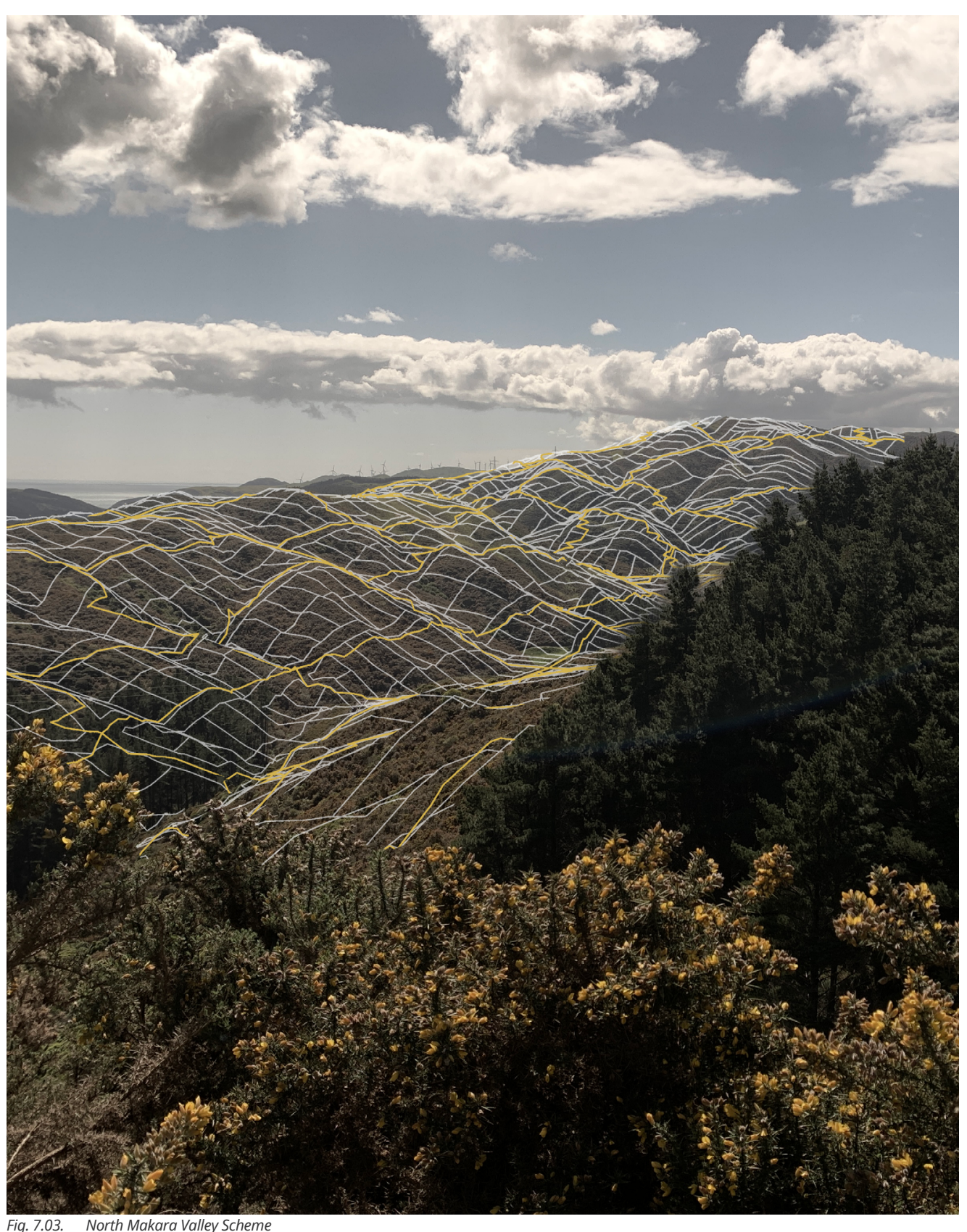




\section{Rapid Concepts}

In order to evaluate the tool's adaptability, quick concept schemes have been generated in the six suburbs covered in Chapter 4's photo journal. These suburbs cover terrain with varying degrees of extremity, with some existing on single slopes and some existing in valleys, therefore being able to showcase both grid generation modes of the tool.

These rapid concepts are not proposals for replacing the entire suburb, and as such do not have designed connections to the existing urban network. They are purely to demonstrate how the tool reacts to different terrain and to use what currently exists as a point of comparison.

As a testament to the tool's efficiency and ease of use, these concepts each took within 20 minutes to generate. Even with a less experienced user, a well resolved scheme with many adjustments to steps along the process should take under an hour.
The outputs have been showcased as road networks with coloured analyses showing road steepness and Betweenness Cen of both the original and concept networks. Statistics are compared between the two schemes, though for a best comparison the area of the original suburb analysed is only the area directly corresponding to the concept scheme, not the entire area as displayed in the graphic. The extra information has been included only as a visual aid to understand the existing layout of the suburb.

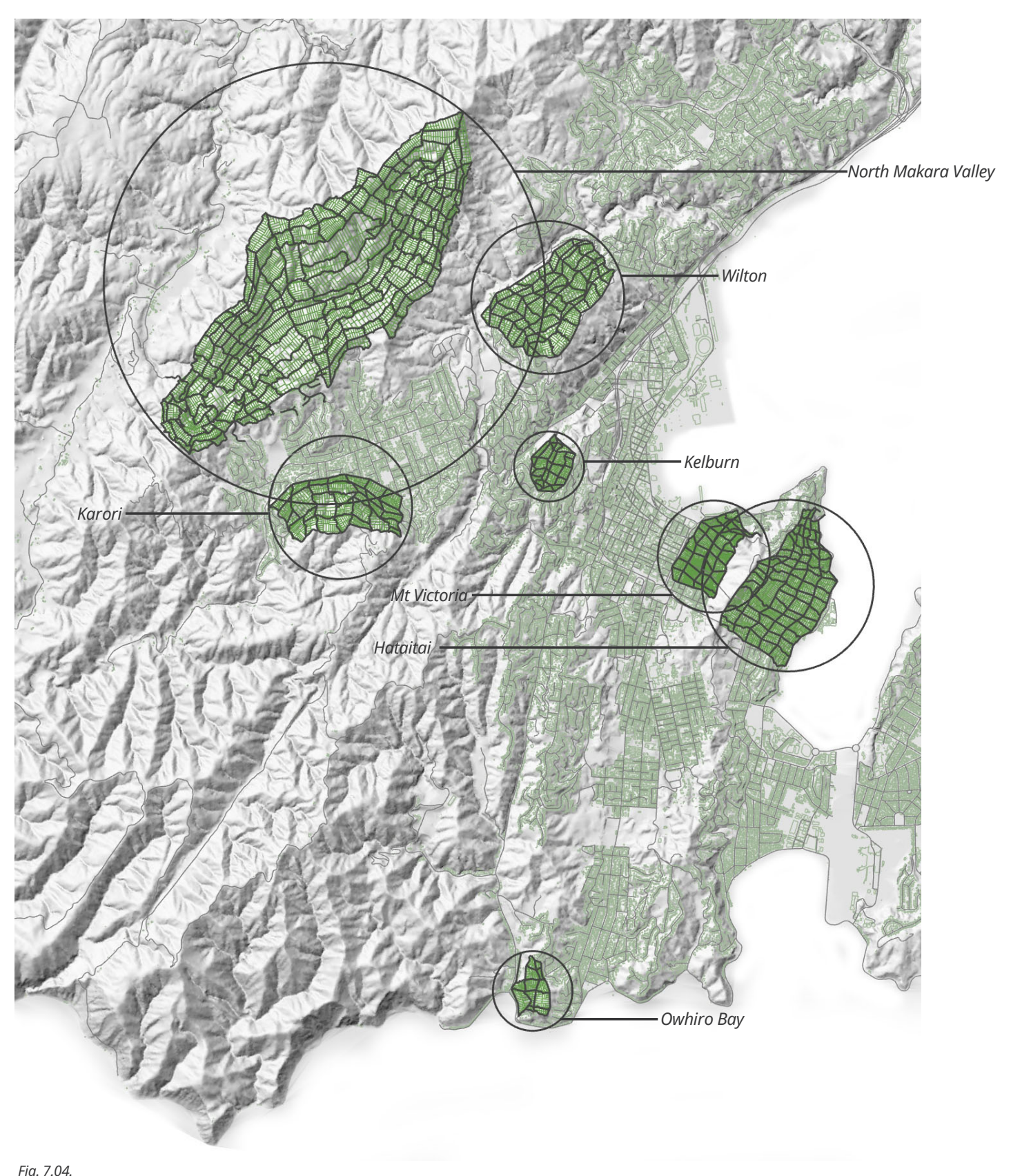



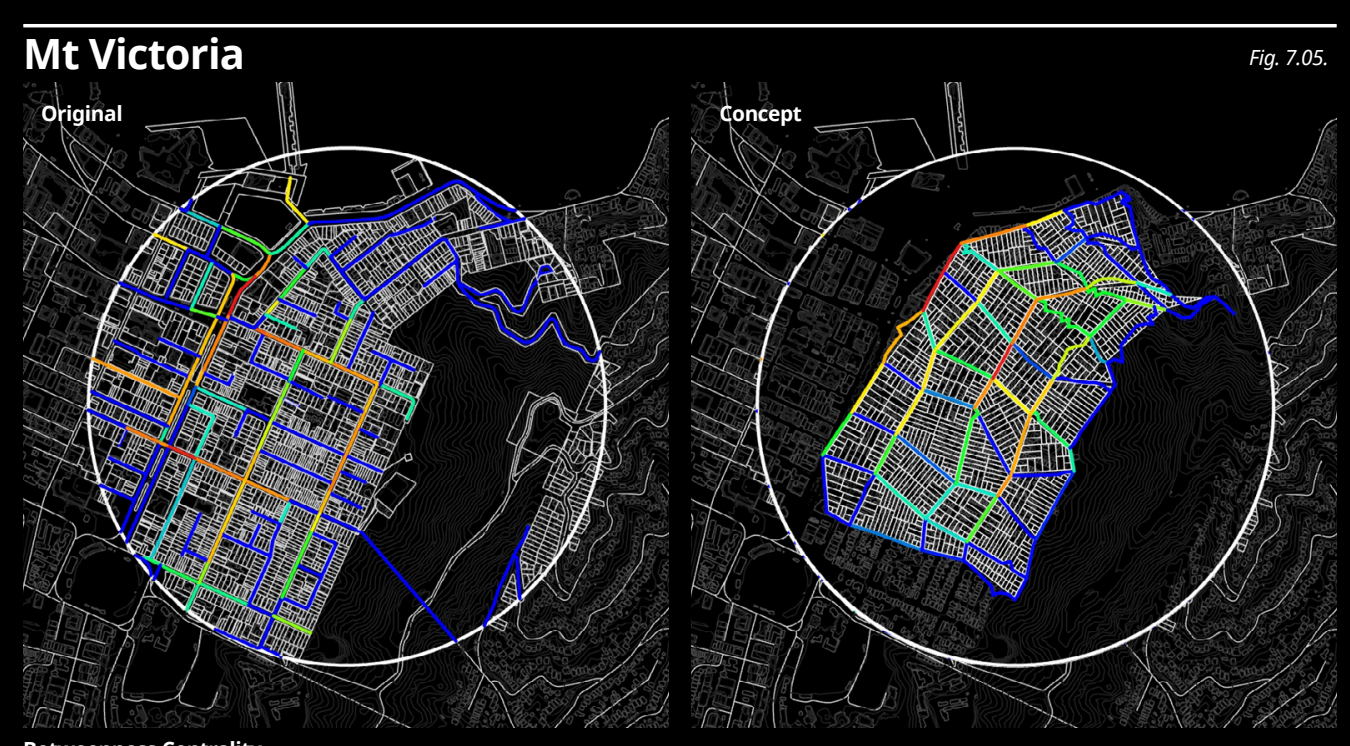

Betweenness Centrality

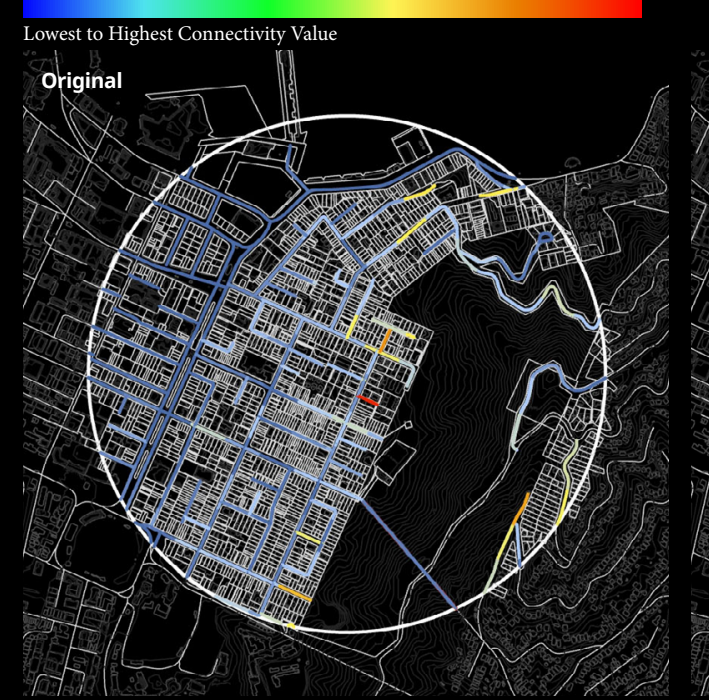

Road Steepness

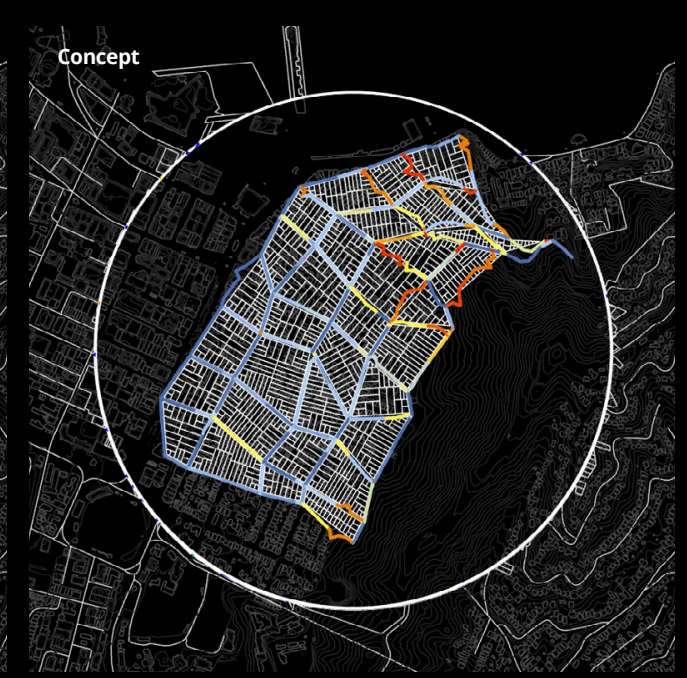

Shallowest to Steepest Road Gradient

Road Length
Original: $7345 \mathrm{~m}$

Number of Parcels

Original: $7345 \mathrm{~m}$
Concept: $11628 \mathrm{~m}$

Original: 1198
Concept: 1014

Average Parcel Size Original: $308 \mathrm{~m}^{2}$
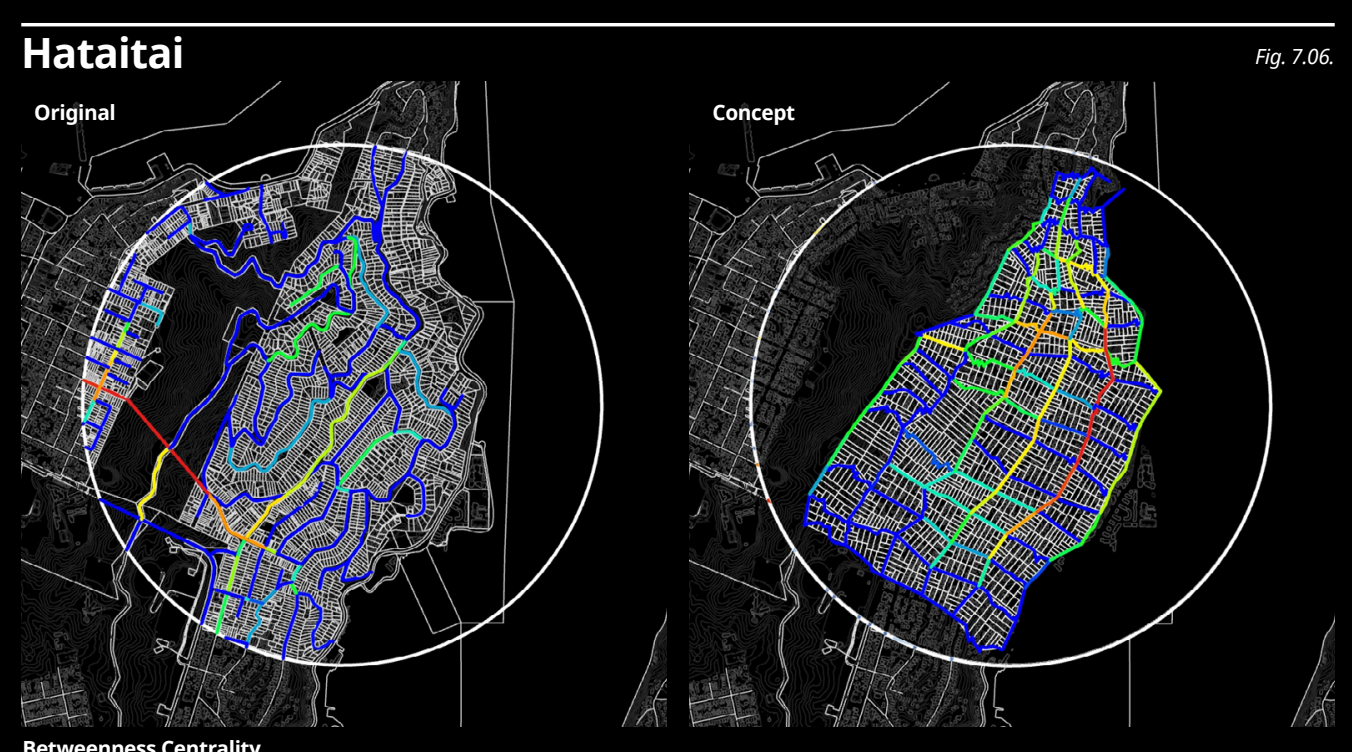

Betweenness Centrality
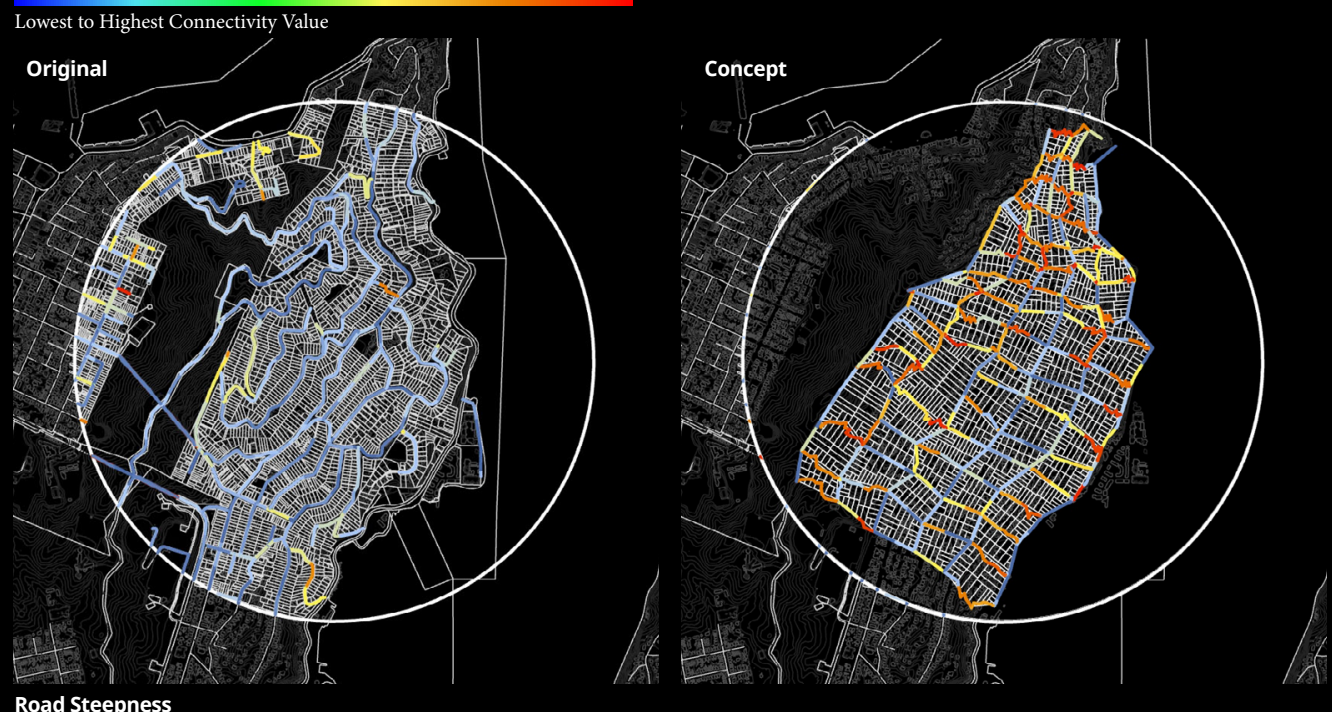

Road Steepness

Shallowest to Steepest Road Gradient

Road Length
Original: $14849 \mathrm{~m}$

Concept: 26169m

Number of Parcels
Originl. 1 1773

Original: 1773
Concept: 1501

Average Parcel Size Original: $522 \mathrm{~m}^{2}$
Concept: $77 \mathrm{~m}^{2}$ 

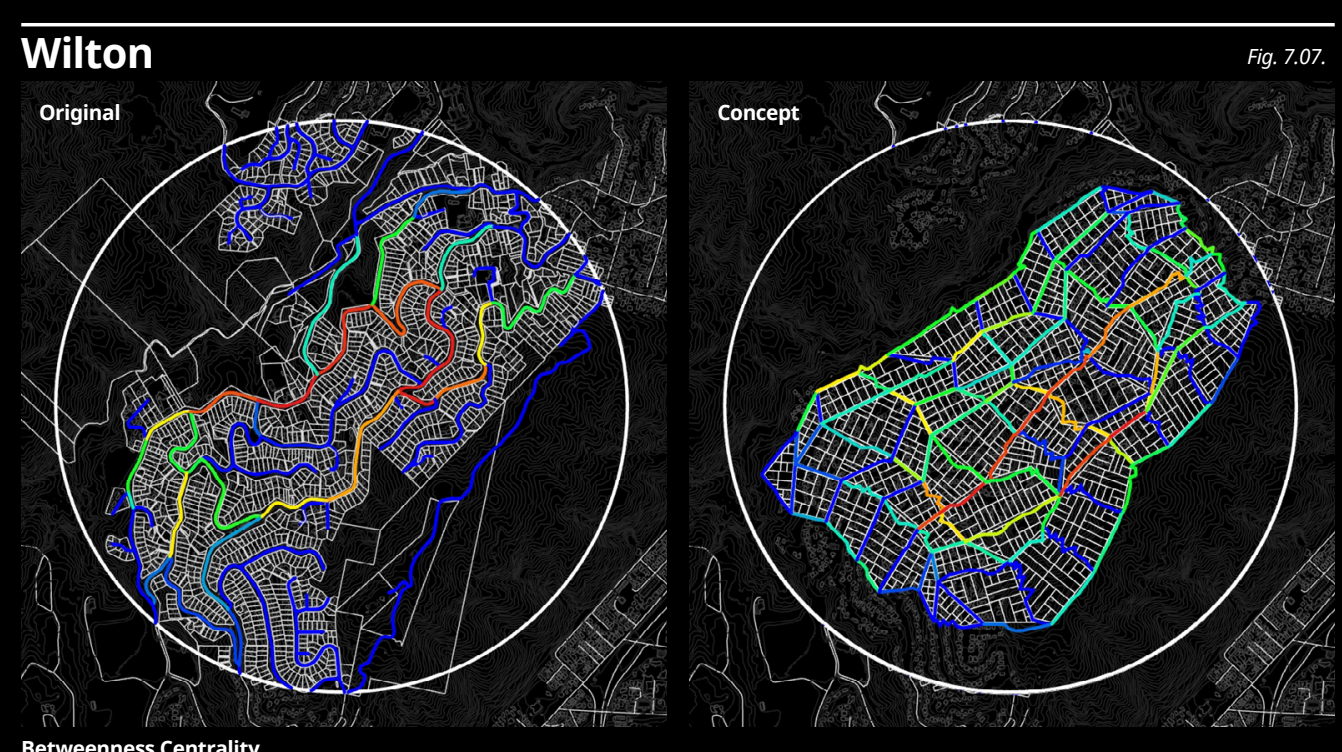

Betweenness Centrality
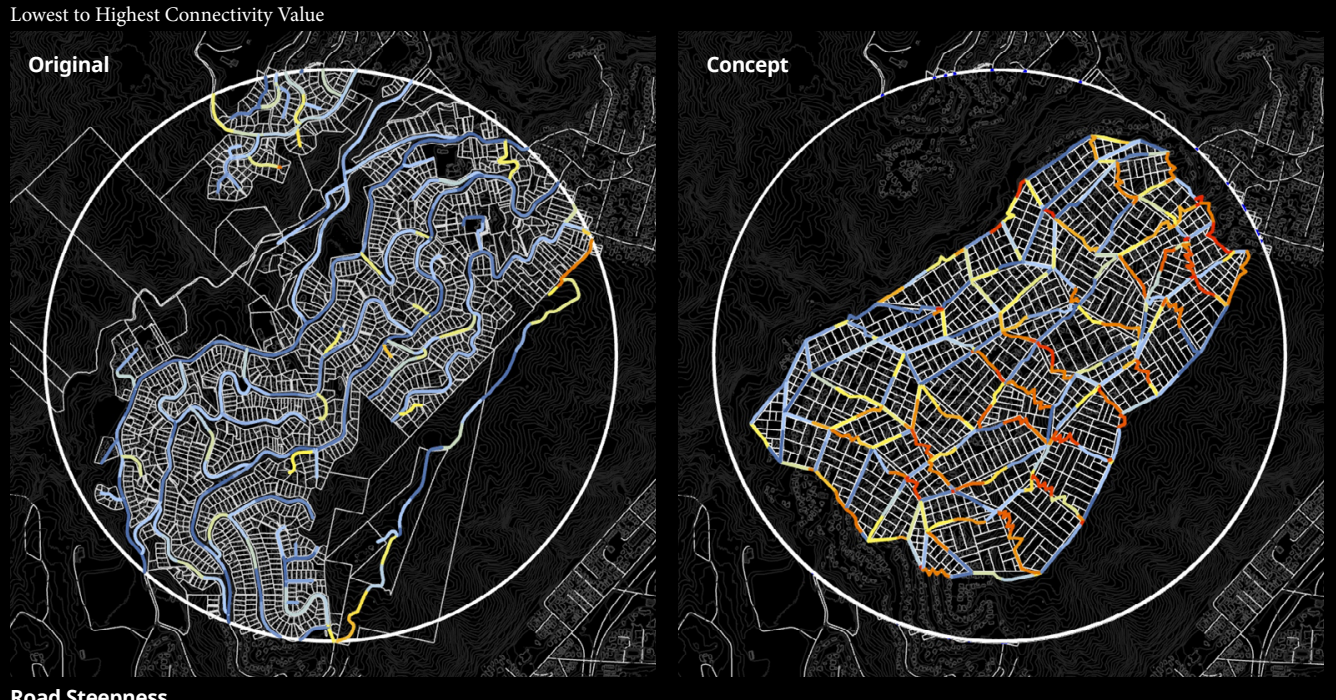

Shallowest to Steepest Road Gradient

Road Length
Original: $10715 \mathrm{~m}$ Number of Parcels
Original: 1062 Concept: $23956 \mathrm{~m}$ Average Parcel Size
Original: $835 \mathrm{~m}^{2}$
Concept: $1032 \mathrm{~m}^{2}$
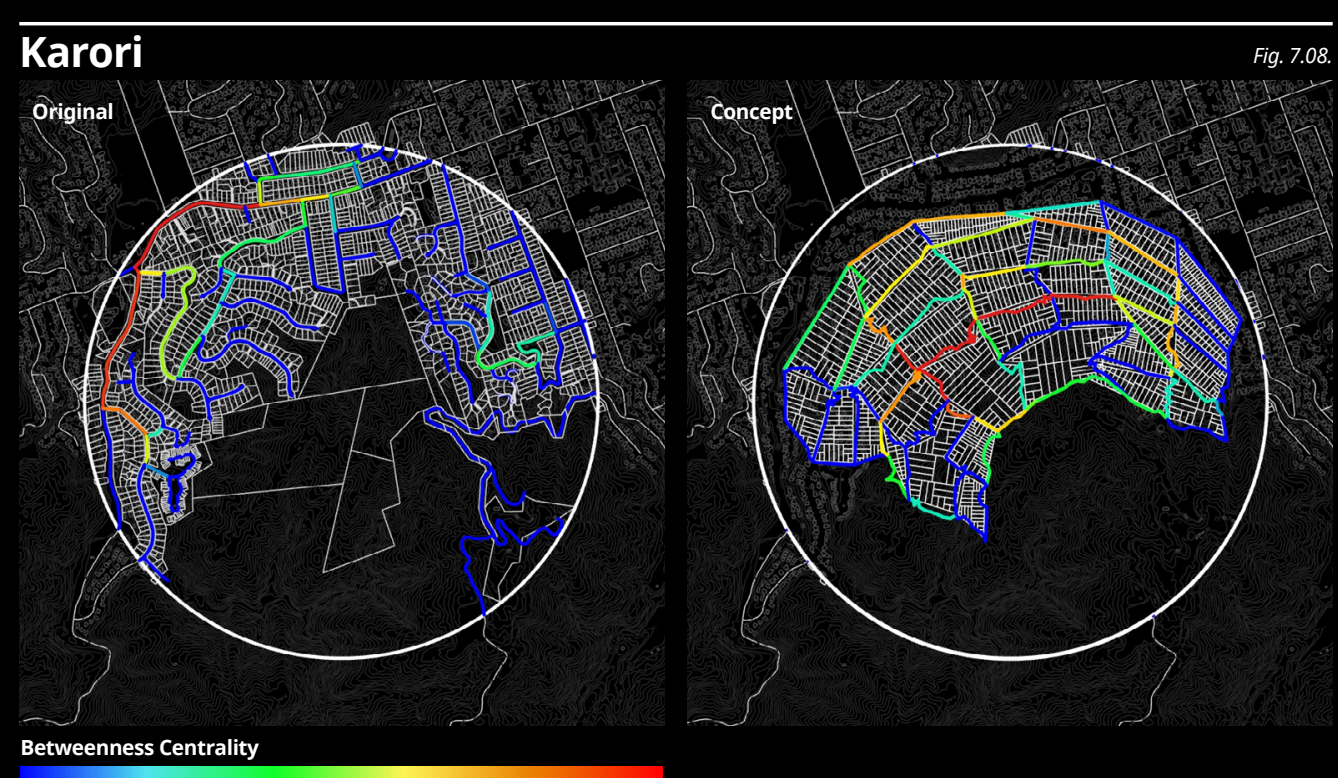

Betweenness Centrality

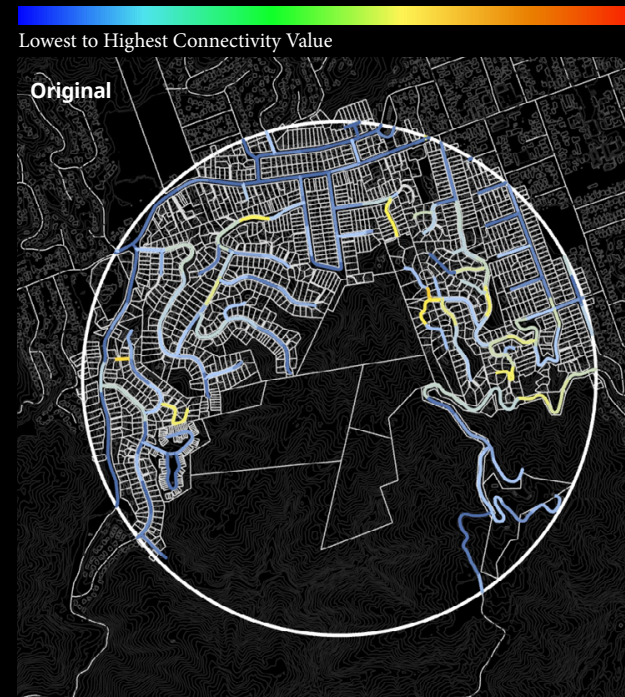

Road Steepness

Shallowest to Steepest Road Gradient

Road Length
Original: $8377 \mathrm{~m}$

Original: $8377 \mathrm{~m}$
Concept: $17327 \mathrm{~m}$

Number of Parcels

Original: 850
Concept: 1250
Average Parcel Size

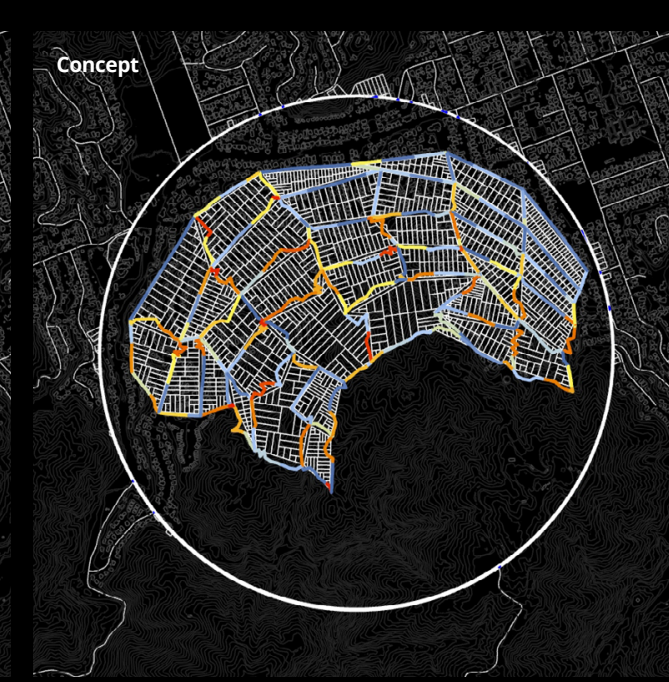
Concept: $635 \mathrm{~m}^{2}$ 

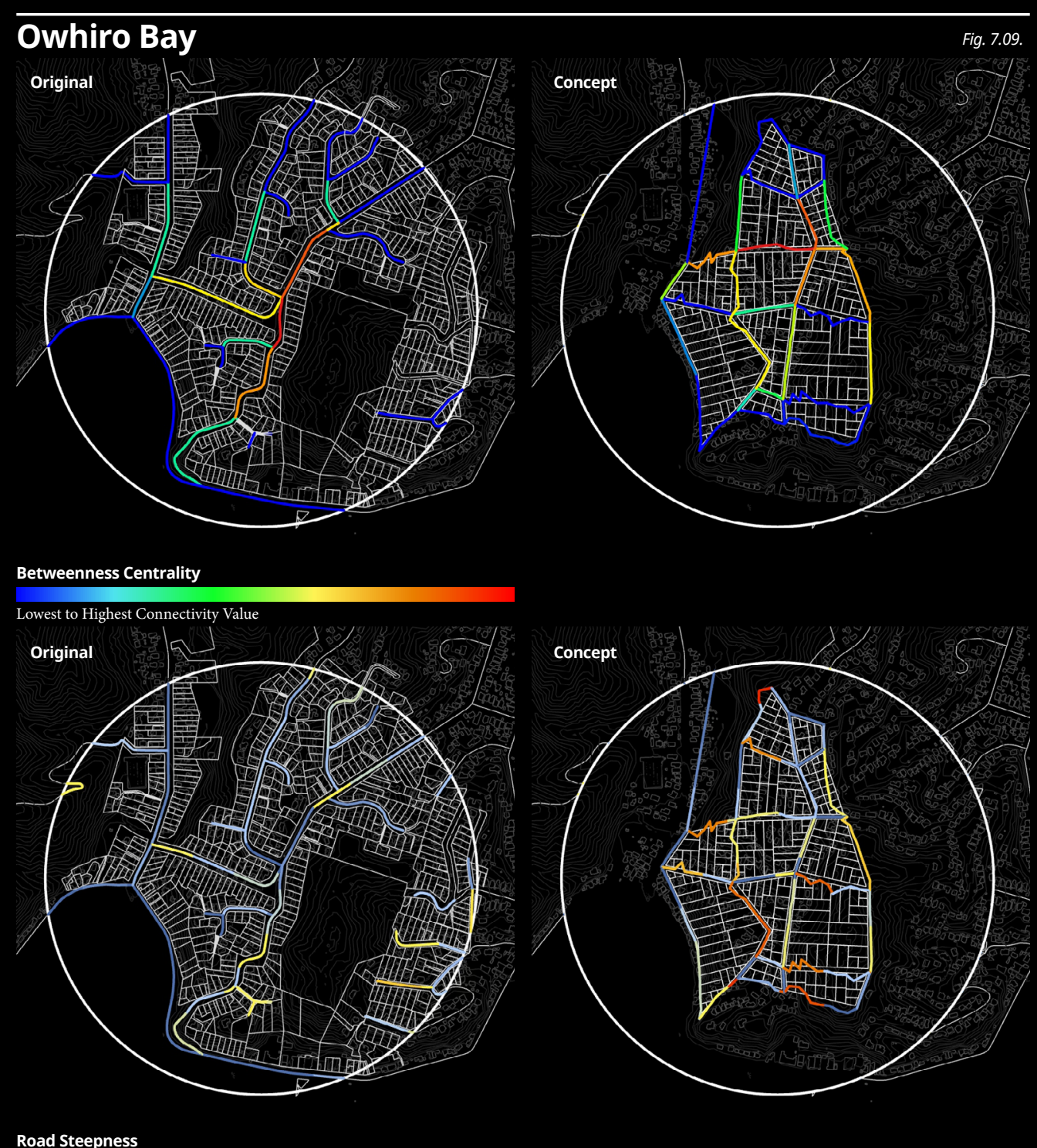

\section{Shallowest to Steepest Road Gradient}

Road Length
Original: $1070 \mathrm{~m}$

Concept: 5114m

\section{Number of Parcels}

Original: 195
Concept: 248
Average Parcel Size Original: $905 \mathrm{~m}^{2}$
Concept: $702 \mathrm{~m}^{2}$

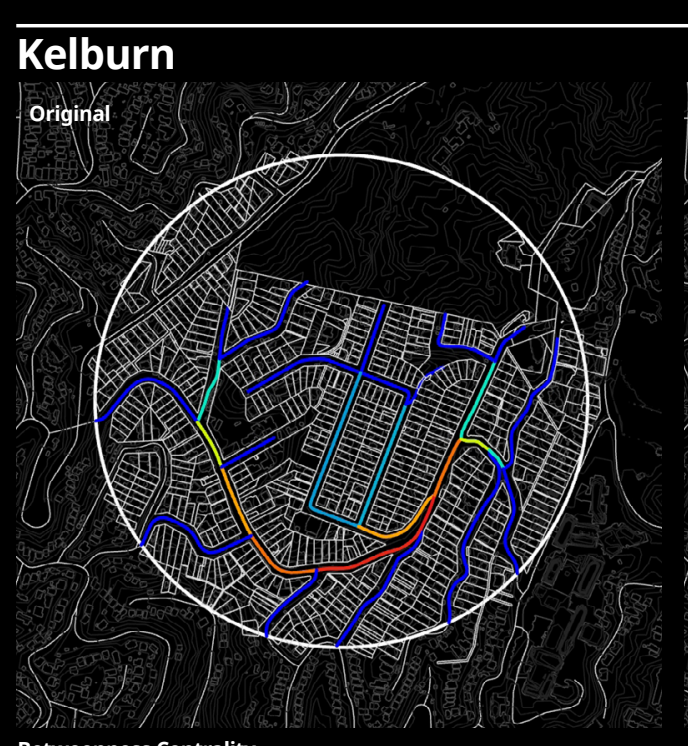

Betweenness Centrality

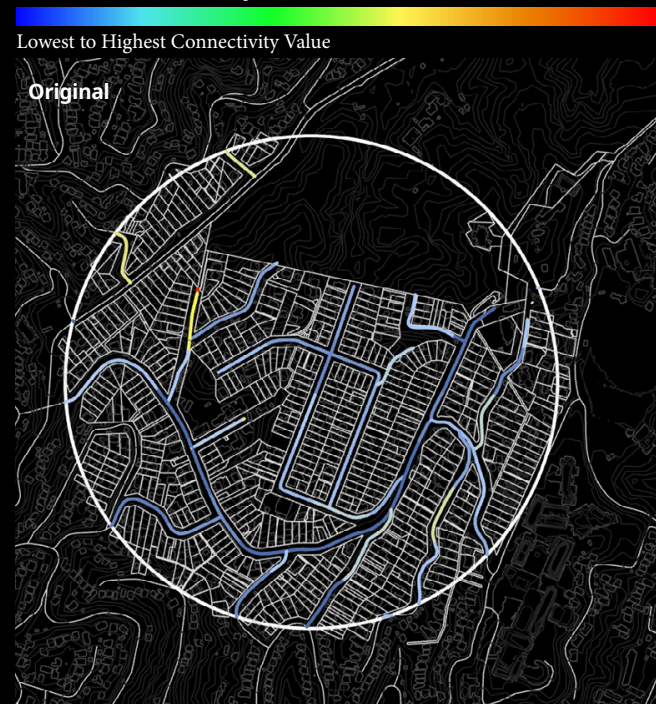

Road Steepness

Shallowest to Steepest Road Gradient

Road Length
Original: 1883m

Original: $1888 \mathrm{~m}$
Concept: $7308 \mathrm{~m}$

Number of Parcels

Original: 334
Concept: 372

Average Parcel Size

Original: $463 \mathrm{~m}^{2}$
Concept $526 \mathrm{~m}^{2}$ 


\section{Rapid Concepts \\ Discussion}

Rather than evaluating how effective the North Makara Valley scheme is as an urban plan, these concepts are helpful in evaluatin the tool itself. By comparing the conceptual to the existing, qualitative statements on what is better or worse can be made.

Before discussing concepts, there are two key caveats on the data to note. Firstly, on the steepness maps, steepness has been averaged over $100 \mathrm{~m}$ lengths. The steepness will appear very high on the concept maps because the land has not had the opportunity for an earthworks to ease out small complications in terrain. On the other hand, the contour lines used to generate the steepness informatio already reflect the earthworks done on the existing roads, widening the difference between the two analyses.

Secondly, there are occasional discrepancie between the total area provided by concep schemes compared against original schemes. This is where the concept schemes have moved over existing green areas, such as the Tow Belt in Mt Victoria or Hataitai, the Botanica Gardens in Kelburn or Burrows Avenue Park in Karori. The aim of this comparison is not to state that the tool can produce more sellable area, it is to show how it interacts with terrain in comparison to existing systems.
The key advantage of the grid system and the main reason it was selected as the central logic of the tool was its role in producing a successful urban circulation network, easing traffic choke points. This is shown clearly in the Betweenness Centrality analysis where the concept scheme roads are much brighter colours across the board, indicating greater connectivity. The key difference between the original and concept schemes is that the brightly coloured lines often follow a single, traceable path in the original scheme, but are spread out along several paths in the concept scheme. This ensures a resilient system where traffic does not build up along a single arterial route.

With that said, there are segments in the generated schemes that are very steep. One of the issues with the tool is that it will always take the most direct route, with no decision making process as to whether to take a less direct but shallower, route. This is partially mitigated by the generative solving algorithm step which attempts to set the tool on the least steep path, but on very steep terrain the tool will still use many switchbacks at the maximum possible angle. This is the central issue of attempting to bring a grid logic to a slope: there must always be a component of the grid moving down the slope, and on the most extreme sites this component is simply too steep. This is a particularly important issue when considering public transport in the tool's schemes, as buses often have more restricted movement than cars and may not be able to tackle the steepest roads or tightest switchbacks.
The logical next step would be to remove the paths that have switchbacks or are too steep, leaving in only a few of the shallowest roads to connect 'row' roads together. To put it in terms of the urban typologies discussed earlier, this moves away from the face-adjusted grid typology to face-adjusted parallel, which seems to work well in Hataitai and Wilton.

By having only a few access points between row roads, connectivity would diminish. In the current state of suburban New Zealand it is hard to imagine this as a significant issue: current suburban density levels see little traffic outside of peak times. If urban density is to be intensified across the board, the issue may become more pertinent.

The attempted implementation of the grid system has an obvious effect on one of the analysed statistics: concept schemes had more roads within them, with the greatest change in the Kelburn concept with a $288 \%$ increase. Again, with the current status quo it is hard to justify the excess roads; developers would likely not find them viable as they reduce sellable land and add to infrastructure costs. This research would assert that the tool's high level of road placement is justified: developing a greenfield site involves a large amount of responsibility as any planning decisions are likely to remain into the distant future. Grid systems are expandable and help to futureproof the scheme for extension purposes, particularly when compared to cul-de-sacs which are incredibly difficult to extend. 


\section{Limitations}

As mentioned at the outset of the thesis, this research has been undertaken without the coding experience or cross-faculty support usually seen in comparable pieces of research. Using tools available within Grasshopper and open-source extensions, the tool manage to achieve what it set out to achieve, but not without caveats and room for improvement.

Tool development and execution was performed on a powerful computer with 16GB of RAM and a recent NVIDIA GTX2080 graphics card. There were times when the compute still struggled to complete some calculations. Frequently, technological constraints create the limitations in the tool's functionality. Recommendations to alleviate computer crashes can be seen among other annotations in the definitions contained in the appendix.

The tool has been split into 6 definitions as seen in Chapter 6 in order to minimise RAM usage at any one time. It is recommended that at the end of each script, the data for each outpu is internalised - a process that saves the dat within the Grasshopper script while preserving its data structure. These internalised data components can then be copied over to the next script, where matching input components are ready to receive their data. This process has been selected instead of baking the information out into the Rhino model as baking results in flattening of data tree structure.
The first limitation has been referred to previously: it is that the agent-based road pathfinder will always take the shortest route possible, regardless of whether it is at the maximum definable steepness. As a set of instructions, the agent cannot make compromises or judgement calls based on if there is a less steep route available. This is mitigated by a few things: firstly, by easing the roads first, the tool attempts to set the agent up for the best possible chance of success by reducing the overall steepness of the road as much as possible. Secondly, by specifying a large enough step size, switchbacks produced by the tool are likely to be more viable. All simulations in this thesis had a step size of $22 \mathrm{~m}$, a length that compromised between possible vehicle and pedestrian uses. The switchbackangle limiting tool was created as an additional form of mitigation, but it can produce some unpredictable results and was not used in generating the showcased schemes.

The second limitation comes in the relationship between the pathfound roads and the overall block shape. In order for the offset component to give width to the generated roads and shape the blocks, the roads themselves have to be simpler shapes due to technological constraints. The simplification process can sometimes result in the actual road overlapping with the neighbouring block, making some parcels considerably smaller than intended. An attempt to remedy this was made by defining the road width as the greatest distance between the simplified road and the actual road, which resulted in unrealistically large road widths but no overlaps. This remediation step was not used in the final script and the end result is that occasionally in very steep areas, there will be an overlap of road and parcel. This does not make a huge difference to the scheme as a whole, and could easily be manually remedied if the concept were carried forward to a more advanced design stage.

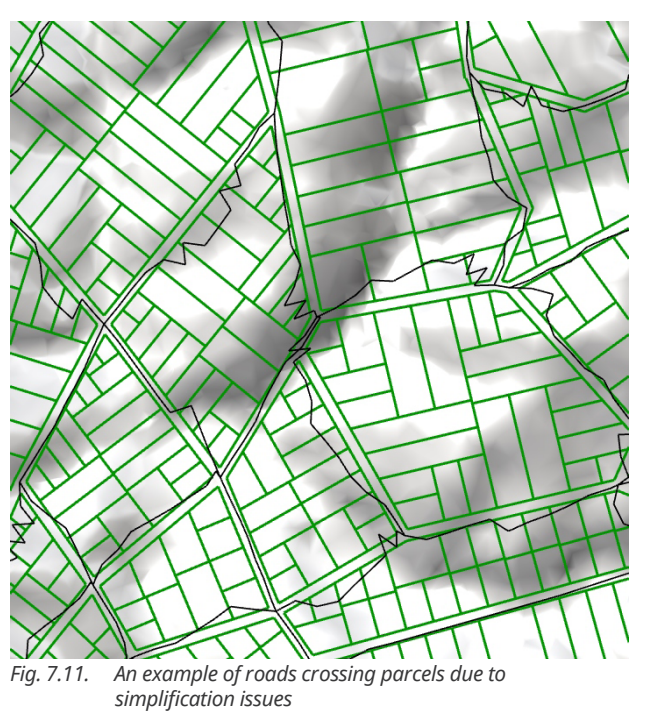

The third limitation for the overall too stems from limitations within an extension. As mentioned in the North Makara Valley Evaluation section, the Parcel component from DeCodingSpaces Toolbox struggles with the size of the scheme. Because the component functions by iteratively dividing the block until it cannot go beyond a minimum constrain (Bielik et al. 2019), if the scheme is too large and still has areas to divide the tool can crash. This issue can be avoided by breaking the schem into sub-areas, which could be advantageous in the largest schemes to improve control of output and create areas of varying density.

\section{Summary}

Though not without flaws and limitations, the review of the schemes has shown that the tool is achieving a valid first step in generative urban design in complex terrain. Its ease of use, speed of development, and ability of the designer to guide the process complement the tool's innate logic and ability to apply it to challenging sites. As an early tool in its specific field, it is well positioned to provide a base for future work 


\section{anters \\ Next Steps}

\section{Modifications to the tool}

Before examining steps that could be taken to expand the tool's functionality and push its schemes beyond the concept stage of design, there are some features that were not able to be added to the tool due to time or complexity. These features would enhance the tool's functionality and reduce the amount of manual input required from the user.

The first step would be seeking to remedy some limitations discussed in the previous chapter. Improving the tool's abilities to ru on lower-end machines and handle more complex calculations of block or parcel limits would greatly benefit the tool's functionality. To properly implement this it is likely that sophisticated knowledge of coding and custom componentry would be necessary.

The second would be a way for the tool to automatically detect the borders of a valley This was attempted through the drainage model test from the Prototypes chapter as well as several scripts that did not produce any meaningful results. This step requires a large amount of human intervention in the form of specifying the key features of the site or trimming the initial output mesh. Reducing the amount of human intervention required would streamline the workflow.

Similarly, the other step that requires a large amount of human intervention is the evaluation of the road network and its modification as suggested by the tool. For this first version of the tool, the decision was made to have users manually review and remove highlighted roads in the Rhino environment in order to incorporate existing roads into the scheme at the same time. In the event that there are no existing roads, it may be faster to have the tool execute this step for the user by automatically removing roads that do not fit the desired criteria. Due to how the road evaluation tools are set up, automating this would be quite easy, using the same lists that highlight roads to cull them.

These three changes would likely be achievable within the Grasshopper environment with more resources or a dedicated purposemade extension. However to truly maximise 
the tool's usefulness, breaking free of the Grasshopper environment would likely be the largest step. Currently, using the tool requires a basic familiarity with Grasshopper to crea mostly viable outputs, with troubleshooting any step of the tool requiring considerably more familiarity. This limits the tool's potentia usergroup to those with that kind of experience - typically those with some kind of recen architecture training. Designers would be the most likely to be able to access the tool, with developers or councils having to contract ou to a design firm to work and interpret the tool.

A standalone program with streamlined use interface, free of Grasshopper but following th same logic and workflow would be the ultimate form of this tool. As mentioned previously, a improved user interface with unified number sliders and toggles could be implemente within the script using Grasshopper extension Human (Heumann 2018), but the decision has been made not to do this. By keepin number sliders in situ in the script, user can more readily follow the workflow, rea troubleshooting or process notes within the script, and enable/disable components relevant times to minimise computer load and avoid system crashes.

\section{Further additions to the scheme}

If development were to continue on this tool, the next step would be to look at how the outputted scheme could carry more information. A good starting point would be to examine land use within the scheme by projecting statistics about population requirements and using that information to allocate areas other than residential to blocks. This feature would require a user-definable density value for housing, potentially based on proximity to a similarly definable urban centre.

From there, it could calculate roughly how many independent households would be supported by the scheme and in turn the community infrastructure required by that populace. This might include schools, local and central shops, substations, public spaces and other amenities. In the context of extreme terrain it makes sense to concentrate these shared spaces where the terrain is flattest in order to ensure adequate accessibility. It would also be logical to plan for local shops to be dispersed throughout the scheme, to enable residents to access essentials without tiring journeys up or down steep terrain.

Other additions that could begin to be considered are:

- Landscape information and soil types for engineering and infrastructure purposes - Drainage, power and internet access
- Earthworks to improve roads

Costs and economic value

The more layers of information that can be added to the end of the workflow improve the viability of the scheme, bringing concepts closer to realisation and making the transition to later design stages easier.

\section{Parallel Research}

This tool's central logic was that of the urban grid, which is a typology frequently seen in urban centres on flat land but only one of several typologies identified on steep sites. If one of the other typologies identified from Wellington's hill suburbs were used, how could it shape the tool? One of the centra issues identified with the grid system was that 'column' roads were inevitably ver steep. What would a typology that created network without relying on regular movemen perpendicular to contour lines look like?

This question could lead to several parallel pieces of research that also seek to find a generative urban planning procedure for complex terrain, focusing on a different typology each time. Parts of Head for the Hills could be incorporated into these tools, an remaining in the Grasshopper environmen would allow them to benefit from the same wealth of plugins and additional analysi Grasshopper can provide.
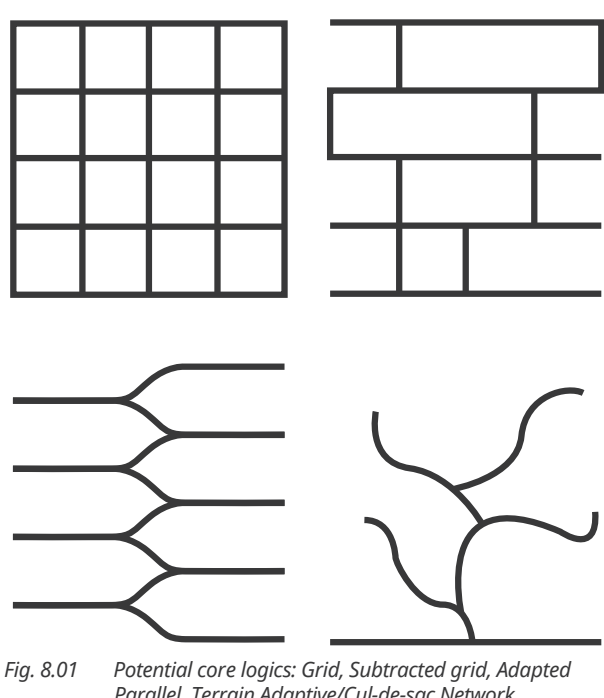

There could also be a difference in approach to the order in which steps in the tool are carried out. Rather than defining an entire area and generating geometry to fill it, one approach could be specifying a starting point and iteratively spreading a solution until the desired threshold is met. There are some promising experiments with this logic (Dennemark et al. 2017) that take similar modular approaches to their workflow within the Grasshopper environment, and could be linked up to aspects of Head for the Hills. 


\section{Chapter 9}

\section{Conclusion \& Critical Reflection}

\section{Conclusion}

Head for the Hills sought to answer th research question "how can generative design improve the way urban networks are designed in complex terrain?" By looking at case studies and qualitatively analysing Wellington's hill suburbs, the key logic of the urban grid was identified, along with other considerations to include in the development of a generative tool. Through iterative Grasshopper steps, this logic was extrapolated into a fully adaptive workflow capable of producing functional urban schemes on steep terrain, requirin steering and review by a human designer. The tool's functionality is explained on a scrip by script basis, and its usage is demonstrated through the detailed scheme in North Makara Valley. Review of the scheme alongside severa rapidly generated schemes through the len of Space Syntax and in comparison with existing urban schemes in complex terrain demonstrated its merits. The answer to the research question is then an affirmative, but that is not to say this thesis comprehensively covers what can be achieved with generative design in complex terrain

Head for the Hills is particularly successful in its ambition to be a tool for generating concepts: the tool is easy to use with appropriate Grasshopper knowledge and can generate solutions, steered by the user, in a rapid timeframe.

In terms of the tool's output, the North Makara Valley scheme is one that is mostly viable and would be resilient in its functionality up to a population far denser than currently seen in Wellington. To be completely viable, the scheme would require strong changes in the way people live in urban spaces and in complex terrain. A public transport network would have difficulty in negotiating the steeper roads, as would older or heavier cars. Pedestrians would spend a lot of time navigating steps and would certainly rely on vehicular transport if they needed to visit a supermarket. As discussed previously, accessibility would remain an issue. 
These traits are unlikely to be intrinsically tied to the site and are more likely to be common in schemes outputted by the tool. Innovation is certainly possible, potentially looking to urban cable car networks (Sokol 2010) or ski resorts as inspiration.

As the level of innovation in generative design improves, the level of change needed in the status quo for inhabitants decreases. Head for the Hills presents only the first step in bringing generative design into this terrain condition and with continuing research and expertise from other architectural and computer science disciplines, could continue to improve. A more robust, more 'intelligent' design system may find a better way to pathfind and avoid overly steep roads. As time passes and the innovation of both designing in and living in complex terrain improves, there may eventually be a intersection where a highly functional scheme can be reached in even the most challenging of areas. Until that point, care needs to be taken that development does not obstruct future possibilities by creating sprawl that continues to see complex terrain as a simple boundary.

\section{Critical Reflection}

To reflect on this thesis, there have been some epistemological and technological difficulties throughout the process, mostly relating to the difficulties of producing a tool as a product of a design thesis.

The need for adaptability in the methodology set at the initiation of the thesis made it difficult to establish a strong evaluative framework for the research. When the methodology was created, it was unknown how smoothly development would proceed, and whether the level of technical knowledge of the author would be sufficient to produce a functional tool. The methodology's adaptability was useful during the tooling stage when there were prototypes that did not proceed as planned. The Drainage Model and Parcel Maker Alpha (as covered in the Prototypes chapter) are two examples of conceptual ideas that could not translate into functional scripts.

While the result is in line with the goal established at the outset, the lack of a strong evaluative framework prevents a quantitative evaluation of how successful the research has been. If the research was to be carried out again, it would be preferable to ensure that there were sufficient technological resources and designer competency to have certainty in the tool completion. This would allow the creation of a more rigid methodology that could have an improved metric for evaluation.
In the tradition of making tools being in the intersection of research for design, research through design, and research about design (Belesky 2018), this thesis has provided a good balance of the three. Investigating the basis for the tool formed the research about design, the implementation of Grasshopper and its variety of plugins formed the research for design, and finally the tooling process and its resultant developed scheme are the pieces of research gleaned through the design process. With that said, having these three separa epistemological ideas has in a way dilute the research. Is the tool, the schemes, or the workflow being evaluated?

Positioning the scheme as the evaluative metric of the tool (through the Space Syntax framework) serves as a good solution to this, but successful urban design is more complex than the circulation network. "Urban design cannot be abstracted from the day-to-day life of urban areas, and all those involved in the creation and functioning of such areas have a role to play in ensuring their success" (Carmona 2003) and so evaluating the true success of a scheme is impossible in abstraction. This research concerns itself with the macro but there is research potential in examining existing worldwide precedents to discover micro qualities of living in complex terrain.

The tooling process explored a great deal of the possibilities within generative design: agent-based solving, genetic algorithm solving, geometry generation from parameters, and human design intervention. There is an element of randomness that is refined (genetic solving algorithms), an element of strict coherence to rules (the agent based solver and its best possible option), and an element of human choice and evaluation when presented with suggestions (highlighting possible problem areas in the scheme). Whether to focus on developing one or the other depends on the level of input the designer seeks. This is valuable for the tool's position as an entry into the niche field and building upon one specific mode of generation may improve its capability in the future.

Through the research process, the area with the most unexpected difficulties was the development of the tool. Due to the complex nature of any advanced computer-assisted design, development of some scripts took unexpectedly long times or ran into softwarerelated obstacles. As alluded to in the Data Structure section in Chapter 6, visualising trees of information as they pass through the tool can be very difficult and can result in scripts simply not functioning. Diagnosing these problems are time-consuming and usually require beginning the script again to approach the goal from a different direction.

Additional complexity comes in the requirement of the tool being totally adaptable. Testing how the tool functions on different sites is difficult, as it is impossible to anticipate every scenario the tool might be exposed to. 
One such issue came up during geometry generation for the Rapid Concept section in Chapter 7: the part of the script responsible for eliminating intersecting roads did not function when the roads in question were generated from straight segments of the valley base curve. This error requires a workaround from the tool user and is annotated within the script, but serves as a good example of how adaptability is very hard to guarantee. Despite this, adaptability is important in the overall functionality of the tool and should not be excluded in future research even at cost of the additional complexity and development time.

True generative design is having the computer generate many alternatives and the designer vetting them (Souza 2020), where the computer does the design and the user is the critic. Genetic solving algorithms embody this method. Agent-based solvers and geometry generation from parameters return agency to the designer by improving their ability to shape the output, rather than simply selecting an output from a large list. The way that the evolutionary solver algorithm was used in Head for the Hills was not as major as it was in comparable papers, opting for only simple calculations in a refinement step. For context, Koenig et al. 2013 used a genetic solving algorithm to generate both street network and land parcels before evaluating, resulting in day-long calculation times. In Head for the Hills, keeping random elements to a minimum gives far more control in the process to the user, which is important when contending with an application for generative design that has not had much exploration. Some may argue that this makes this process not 'true' design, bu along the progress of the tool enabled a more reflective process that could catch potential problems and improve the reliability of the tool on different sites.

Despite the few difficulties in framing the research and producing the tool, still presents a strong first step into application of generative design to complex terrain, and future research could utilize a narrower methodology in order to produce more focused and quantifiable findings.

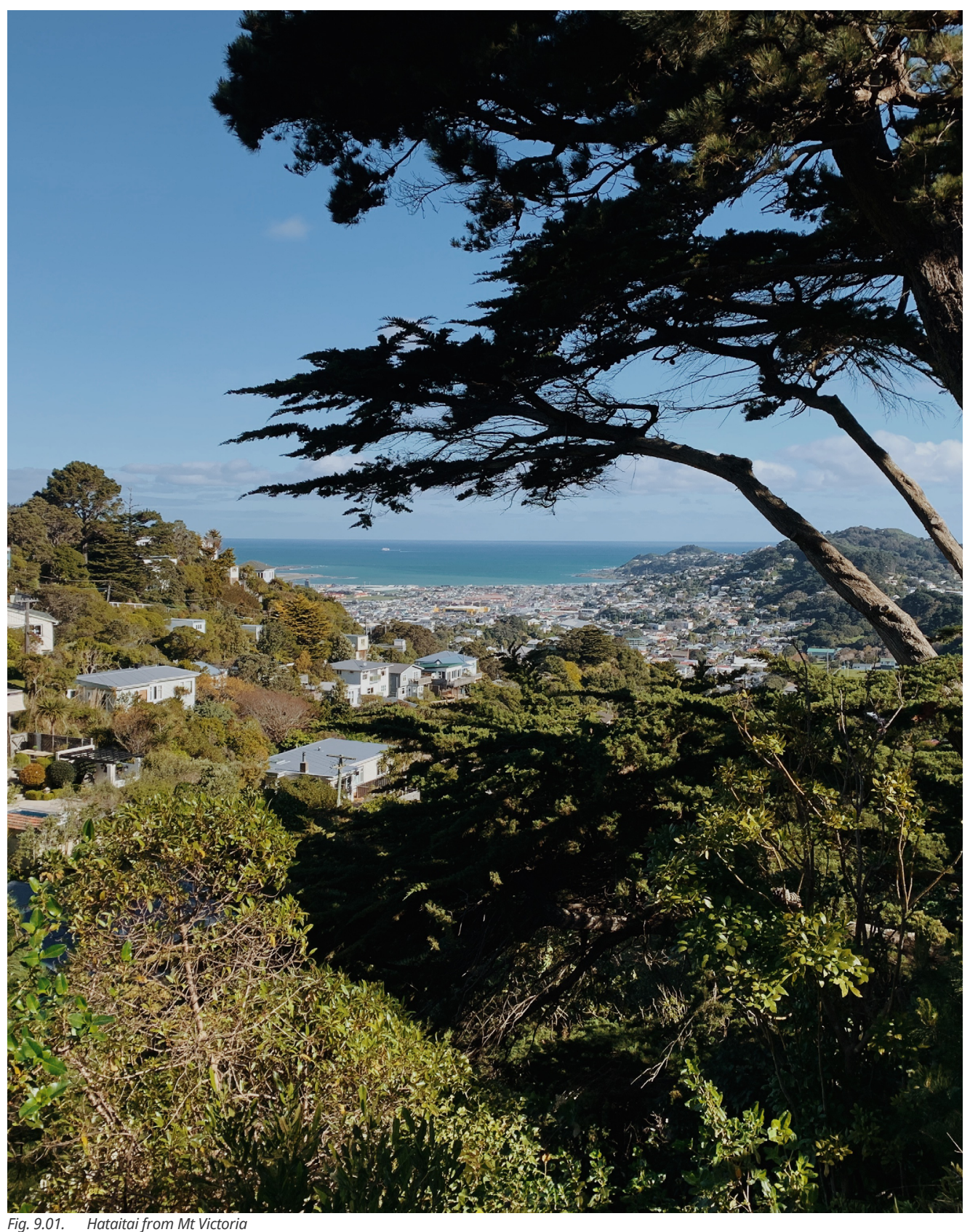




\section{Bibliography}

Abrahamse, Wokje, Keriata Stuart, and Karen Witten. Growth Misconduct?: Avoiding Spraw \& Improving Urban Intensification in New Zealand. Auckland, N.Z: Steele Roberts, 2011.

Ackerly, Duncan et al. "Estimation of Regional Departures from Global-Average Sea-Level Rise around New Zealand from AOGCM Simulations." Weather and Climate 33 (2013).

Adams, D. Urban Planning and the Development Process. London: UCL Press, 1994.

Alexander, Christopher. "A City is not a Tree." Architectural Forum 122 (April 1965): 22

Bahrami, Amin. "Heteroptera.” Plugin. Food4Rhino, November 17, 2014.

Beirão, José. "CItyMaker: Designing Grammar for Urban Design.” CreateSpace Independent Publishing Platform, 2012

Beirão, José, and José P. Duarte. "Generic Grammars for Design Domains.” AI EDAM 32 no. 2 (May 2018): 225-39.

Belesky, Philip. “Testing Terrain: Exploring the Computational Design of Natural Systems in Landscape Architecture." RMIT University, 2018
Bell, R G, R Paulik, and S Wadwha. "National and Regional Risk Exposure in Low-Lying Coastal Areas: Areal Extent, Population, Buildings and Infrastructure.” NIWA, 2015.

Bentley, I. Urban Transformations: Power, People and Urban Design. London: Routledge, 1999.

Bielik, Martin, Reinhard Koenig, and Yufan Miao. "SSS12 Workshop | Parametric Urban Planning with Space Syntax and DeCodingSpaces-Toolbox for Grasshopper." DeCodingSpaces, July 3, 2019.

Carmona, Matthew, Claudio De Magalhães, and Michael Edwards. "Stakeholder Views on Value and Urban Design.” Journal of Urban Design 7, no. 2 (June 2002): 145-69.

Carmona, Matthew, Steve Tiesdell, Tim Heath, and Taner Oc. Public Places, Urban Spaces: The Dimensions of Urban Design. Routledge, 2003.

Conzen, M P. "Alnwick: A Study in Town Plan Analysis." Transactions of the Institute of British Geographers 27, no. 1 (1960).

Cooke, Alice. “Paper Space.” Victoria University of Wellington, 2020.

craft ai. "The Maturity of Visual Programming.” craft ai, September 2015.

Dalley, Emily. “Up The Guts.” Victoria University of Wellington, 2020.
Delaunay, Boris. "Sur La Sphère Vide. A La Mémoire de Georges Voronoii." Bulletin de l'Académie Des Sciences de l'URSS. Classe Des Sciences Mathématiques et Na 6 (1934): 793-800.

Dennemark, Martin, Sven Schneider, Reinhard Koenig, Abdulmalik Abdulmawla, and Dirk Donath. "Towards a Modular Design Strategy for Urban Masterplanning." In City Modelling Tools, 1:10, 2017.

Duarte, José. "Unveiling the Structure of the Marrakech Medina: A Shape Grammar and an Interpreter for Generating Urban Form." AI EDAM 21, no. 4 (September 19, 2007): 317-49.

Fischer, Thomas. "Designing (Tools (for Designing (Tools (For ...)))).” RMIT University, 2008

Fotiadis, Peter. "Mesh Check and Reduce," Grasshopper Forums, March 30, 2017.

Freeman, Linton C. "A Set of Measures of Centrality Based on Betweenness." Sociometry 40, no. 1 (1977): 35-41.

Fugier, Dale. "Grasshopper project curves on mesh surface.” Forum Post. McNeel Forum, April 25, 2018.

Glanville, Ranulph. "CAD Abusing Computing." In CAAD Instruction: The New Teaching of an Architect? Barcelona, 1992

_. "Variety in Design." Systems Research 3, no. 11 (1994)
Global Research. "Planning for Growth Growth Scenario Engagement.” June 14, 2019.

Guy, S. "Developing Alternatives: Energy, Offices and the Environment." International Journal of Urban and Regional Research 22, no. $2(1998)$.

Heumann, Andrew. "Human.” McNeel Forum, March 2018.

Hillier, B, A Turner, Tao Yang, and H Park. "Metric and Topo-Geometric Properties of Urban Street Networks.” Istanbul, 2007.

Hillier, Bill, and Julienne Hanson. The Social Logic of Space. Cambridge: Cambridge University Press, 1984

Hiteca. "Ghshp." Plugin. Food4Rhino, August 26, 2017.

Hunt, Tom. “Wellington's Steepest Streets Revealed and They're Not for the FaintHearted." Dominion Post. August 6, 2019.

Hurkxkens, Ilmar, and Mathias Bernhard. Computational Terrain Modeling with Distance Functions for Large Scale Landscape Design. Wichmann Verlag, 2019.

Kebbel, Sam, and J Ombler. "Nudging toward Urban Walkability in a Car-Dependent New Zealand Neighbourhood." Designing for Health \& Wellbeing: Home, City, Society, 2018. 
Kilkelly, Michael. "5 Ways Computationa Design Will Change the Way You Work." ArchSmarter, April 5, 2016.

Koenig, Reinhard, Yufan Miao, Katja Knecht, Peter Buš, and Mei-Chih Chang. "Interactive Urban Synthesis Computational Methods for Fast Prototyping of Urban Design Proposals." In Computer-Aided Architectural Design

Future Trajectories. Springer, 2017.

Koenig, Reinhard, Lukas Treyer, and Gerhard Schmitt. "Graphical Smalltalk with My Optimization System for Urban Planning Tasks." In ECAADe: Computation and Performance, Vol. 2, 2013: 1-9.

Lang, J. Urban Design, The American Experience. New York: Van Nostrand Reinhold 1994

Levin, Arthur H. Hillside Building: Design and Construction Santa Monica: Art + Architecture Press, 1991.

LGWM. "Let’s Get Wellington Moving -

Context," May 2019.

Lock, D. "The Developer and the Urban Desig Process.” Urban Design Quarterly, April 1993.

McKenzie, Peter. "Welcome to Mouldy-Wood, Aotearoa." The Spinoff, October 7, 2020.
Miao, Yufan, Reinhard Koenig, Katja

Knecht, Kateryna Konieva, Peter Buš, and

Mei-Chih Cha

Design Prototyping: Interactive Planning Synthesis Methods-a Case Study in Cape Town." International Journal of Architectura Computing 16, no. 3 (September 1, 2018): $212-26$

New Zealand Geographic Board. "NZGB Gazetteer | North Mākara Stream.” Land Information New Zealand, April 15, 2013.

Oudshoorn, Nelly, Els Rommes, and Marcelle Stienstra. "Configuring the User as Everybody Gender and Design Cultures in Information and Communication Technologies." Science, Technology, \& Human Values 29, no. 1 (January 1, 2004): 30-63.

Piacentino, Giulio. "Weaverbird - Topologica Mesh Editor." Giulio Piacentino (blog), Augus $31,2009$.

Pope, Albert. Ladders. New York: Princeton Architectural Press, 1996.

Pryor, Michael. "Pufferfish.” Plugin. Food4Rhino, October 17, 2017.

Punter, John. "Urban Design as Public Policy: Evaluating the Design Dimension of Vancouver's Planning System." International Planning Studies 7, no. 4 (November 2002): 265-82.
QGIS. "QGIS: A Free and Open Source Geographic Information System.” QGIS. Accessed September 3, 2020

Rahimian, Mina, José Nuno Beirão, José Pinto Duarte, and Lisa Domenica Iulo “A GrammarBased Generative Urban Design Tool

Considering Topographic Constraints," 267-76. Porto, 2019.

Rhodes-Robinson, Jacob "Bits \& Pieces: Investigating the procedural ideation of architectural massing for early stage design." Victoria University of Wellington, 2018.

Richards, Michael Bradley. "A City Is Not a Tree.” Victoria University of Wellington, 2017.

RICS \& DoE. Quality of Urban Design: A Study on the Involvement of Private Property Decision-Makers in Urban Design. London: Royal Institute of Chartered Surveyors, 1996.

Rowley, Alan. "Private-property Decision Makers and the Quality of Urban Design." Journal of Urban Design 3, no. 2 (June 1998): 151-73.

Rutten, David. "Evolutionary Principles Applied to Problem Solving.” Grasshopper Forums, September 25, 2010.

Ryan, Michael. Toehold on a Harbour. Documentary, 1966.
Sadeghipour Roudsari, Mostapha, and Michelle Pak. "Ladybug: A Parametric Environmental Plugin for Grasshopper to Help Designers Create an EnvironmentallyConscious Design.” Lyon, 2013.

Saris, Milla. "Designing a Sustainable, Medium Density Housing Solution That Is an Affordable Alternative for Developers." Victoria University of Wellington, 2017.

Sokol, David. “Over Site: How Caracas's New Cable-Car System Is Making the City's Favelas More Visible.” Architonic (blog), July 29, 2010.

Soler, Vicente. "Drainage Direction Script." Grasshopper Forums, July 7, 2012.

Southworth, Michael, and Eran Ben-Joseph. "Street Standards and the Shaping of Suburbia." Journal of the American Planning Association 61, no. 1 (March 31, 1995): 65-81.

Souza, Eduardo. "How Will Generative Desig Impact Architecture?” ArchDaily (blog), April 23, 2020.

Stasiuk, David. "Treesloth - Bespoke Geometry." Bespoke Geometry (blog), July 7, 2015.

Stats NZ "New Zealand in the OECD" Stats NZ 2005

. "New Zealand's Population Passes 5 Million.” Stats NZ, May 18, 2020. 


\section{List of Figures}

Stiny, George, and James Gips. "Shape

Grammars and the Generative Specification of

Painting and Sculpture.” Leicester, 1971.

VanDerSys, Keith. "Path Finding across a

Terrain.” Web Forum. Grasshopper Forums,

January 1, 2017.

Varela, Pedro de Azambuja. "Multiple Split

Curves or Shatter All." Script. Pedro de

Azambuja Varela (blog). Accessed November

8,2020

Verniz, Debora, and José Pinto Duarte. "Santa

Marta Urban Grammar,” 477-84. Rome, 2017.

Wellington City Council. "Code of Practice for Land Development Part C,” December 2012.

_ . "Road Encroachment and Sale Policy," September 2011.

—. "Spatial Plan Draft Summary,” August 25, 2020.

"Wellington Urban Growth Plan 2014 2043,” June 2015.

Wenden, Matthew. "Revisiting the Hillside:

Organic, Aggregative Medium Density Housing

in a Wellington Hillside Environment."

Victoria University of Wellington, 2016.

Zwierzycki, Mateusz. “Anemone.” Plugin.

Food4Rhino, December 4, 2013.
All figures not attributed are author’s own.

Fig. 0.01 .

Fig. 0.02 .

Fig. 0.03 .

Fig. 1.01.

Fig. 2.01. Adapted from Richards, Michael Bradley. "A City Is Not a Tree." Victoria University of Wellington, 2017.

Fig. 2.02. Ryan, Michael. Toehold on a Harbour. Documentary, 1966.

Fig. 3.01.

Fig. 3.02. Adapted from Carmona, Matthew, Claudio De Magalhães, and Michae

Edwards. "Stakeholder Views on

Value and Urban Design.” Journal of Urban Design 7, no. 2 (June 2002)

Fig. 3.03. Duarte, José. "Unveiling the Structure of the Marrakech

Medina: A Shape Grammar and

an Interpreter for Generating

Urban Form." AI EDAM 21, no. 4

(September 19, 2007): 330.

Fig. 3.04. Verniz, Debora, and José Pinto

Duarte "Santa Marta Urban

Grammar,” 483. Rome, 2017.
Fig. 3.05. Rahimian, Mina, José Nuno Beirão, José Pinto Duarte, and Lisa Domenica Iulo. "A GrammarBased Generative Urban Design Tool Considering Topographic Constraints,” 269. Porto, 2019.

Fig. 3.06. Rahimian, Mina, José Nuno Beirão, José Pinto Duarte, and Lisa Domenica Iulo. "A GrammarBased Generative Urban Design Tool Considering Topographic Constraints,” 275. Porto, 2019.

Fig. 3.07. Adapted from Koenig, Reinhard, Lukas Treyer, and Gerhard Schmit. "Graphical Smalltalk with My

Optimization System for Urban Planning Tasks." In ECAADe:

Computation and Performance, Vol. 2, 2013: 5-6.

Fig. 3.08. Miao, Yufan, Reinhard Koenig, Katja Knecht, Kateryna Konieva, Peter Buš, and Mei-Chih Chang. "Computational Urban Desig Prototyping: Interactive Planning Synthesis Methods-a Case Study in Cape Town." International Journal of Architectural Computing 16, no. 3 (September 1, 2018): 222.

Fig. 4.01.

Fig. 4.02.

Fig. 4.03 .

Fig. 4.04 .

Fig. 4.05.

Fig. 4.06 . 
Fig. 4.07.

Fig. 4.08. Rahimian, Mina, José Nuno Beirão, José Pinto Duarte, and

Lisa Domenica Iulo. "A Grammar-

Based Generative Urban Design

Tool Considering Topographic

Constraints,” 269. Porto, 2019

Fig. 4.09.

Fig. 4.10.

Fig. 4.11.

Fig. 4.12.

Fig. 4.13.

Fig. 4.14.

Fig. 4.15.

Fig. 4.16.

Fig. 4.17.

Fig. 4.18.

Fig. 4.19.

Fig. 4.20.

Fig. 4.21.

Fig. 4.22.

Fig. 4.23.

Fig. 4.24 .

Fig. 4.25 .

Fig. 4.26 .

Fig. 4.27.

Fig. 4.28.

Fig. 4.29 .

Fig. 5.01.
Fig. 5.02.

Fig. 5.03.

Fig. 5.04.

Fig. 5.05.

Fig. 5.06.

Fig. 5.07.

Fig. 5.08.

Fig. 5.09.

Fig. 5.10.

Fig. 6.01.

Fig. 6.02 .

Fig. 6.03. Gear icon courtesy of Flaticon.com

Fig. 6.04. Gear icon courtesy of Flaticon.com

Fig. 6.05. Gear icon courtesy of Flaticon.com

Fig. 6.06. Gear icon courtesy of Flaticon.com

Fig. 6.07.

Fig. 6.08.

Fig. 6.09.

Fig. 6.01 .

Fig. 7.01.

Fig. 7.02.

Fig. 7.03.

Fig. 7.04.

Fig. 7.05.

Fig. 7.06.

Fig. 7.07.

Fig. 7.08.

Fig. 7.09.
Fig. 7.10.

Fig. 7.11.

Fig. 8.01.

Fig. 9.01. 


\section{Appendix}

Head for the Hills: Computation Urban Design Prototyping for Complex Terrain Part One: GIS Base Outputs

Input: Existing Building Footprints

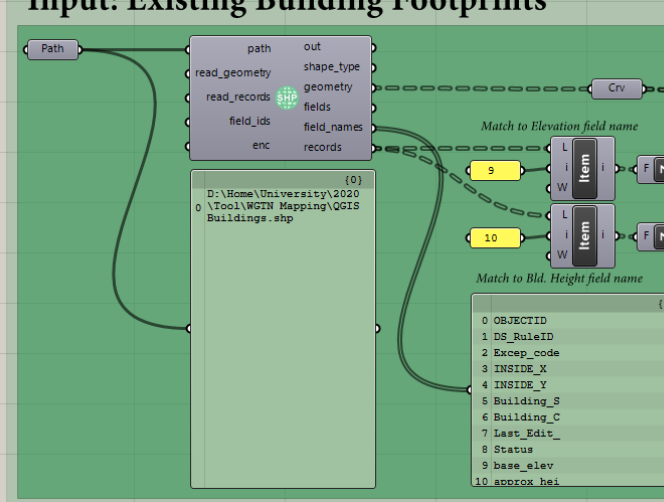

Input: Contour Lines

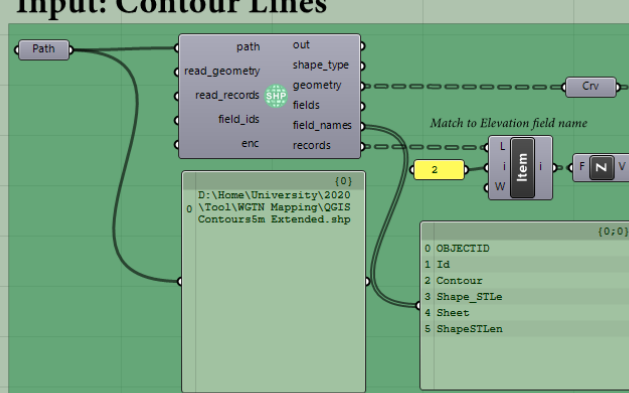

Input: Existing Road Centrelines

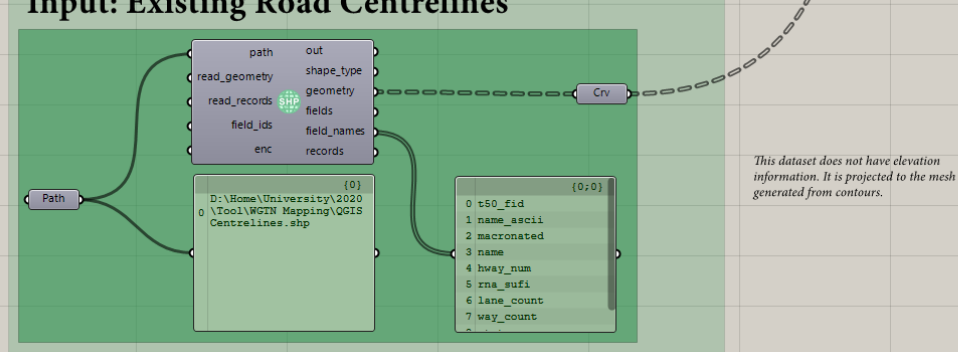

Scaling and Centering

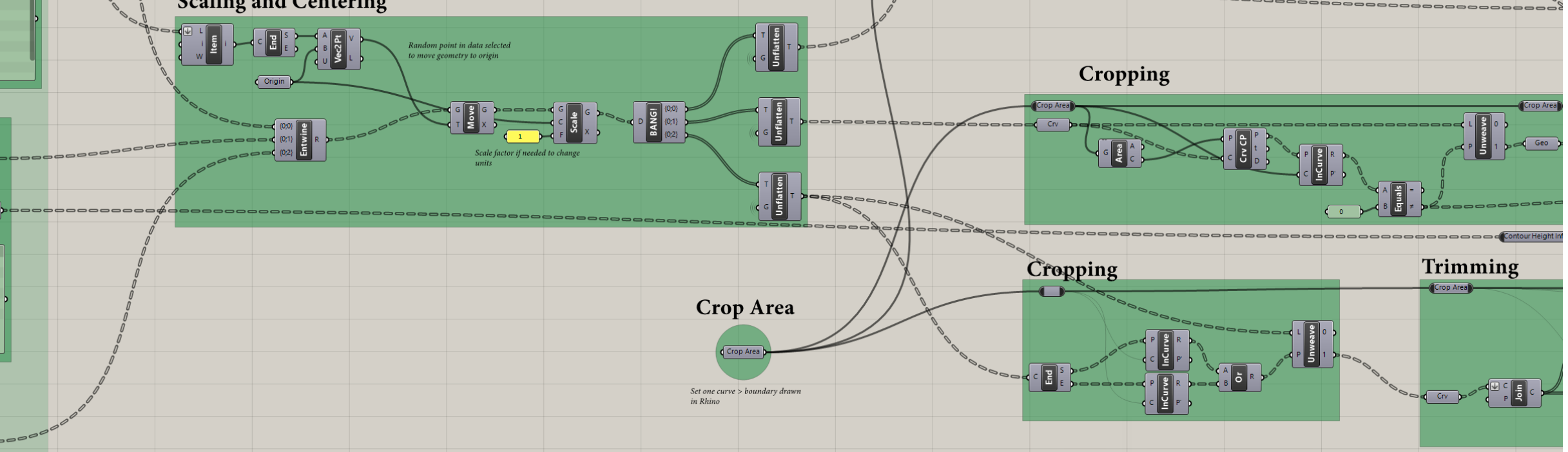



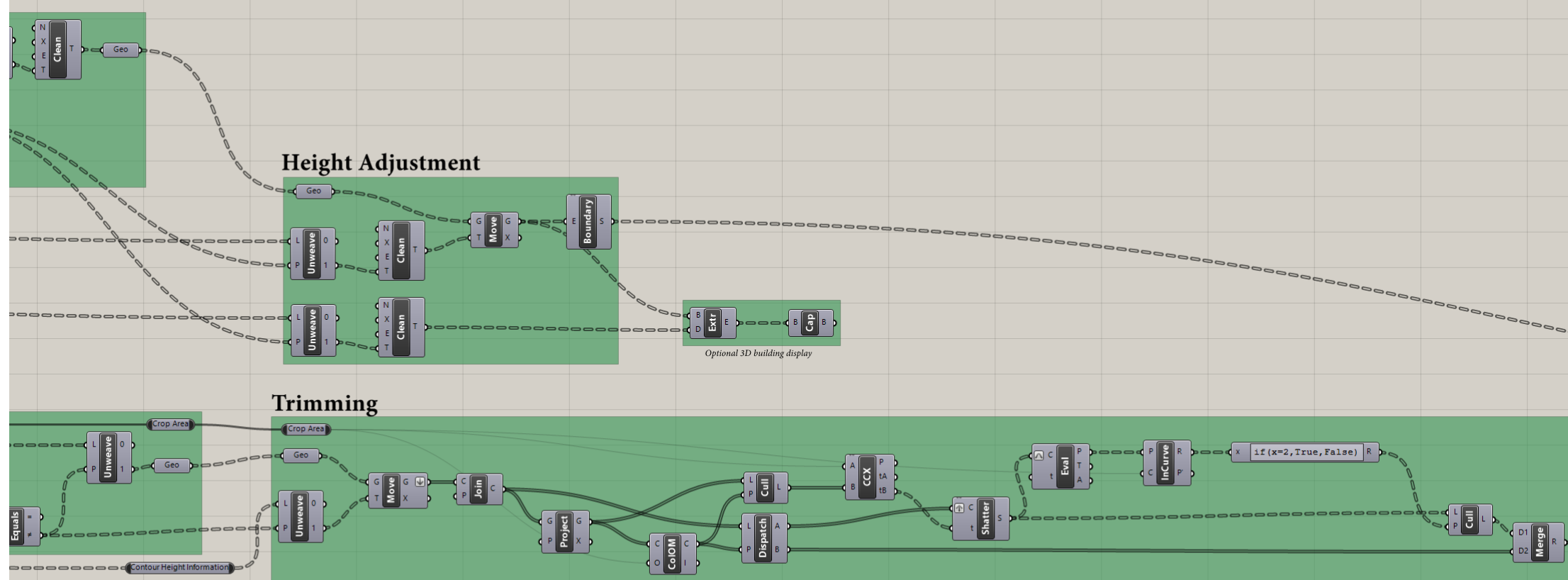

Trimming

Irimming

Mesh Making

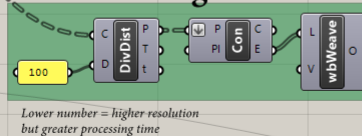

Project to Mesh

-

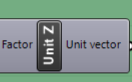




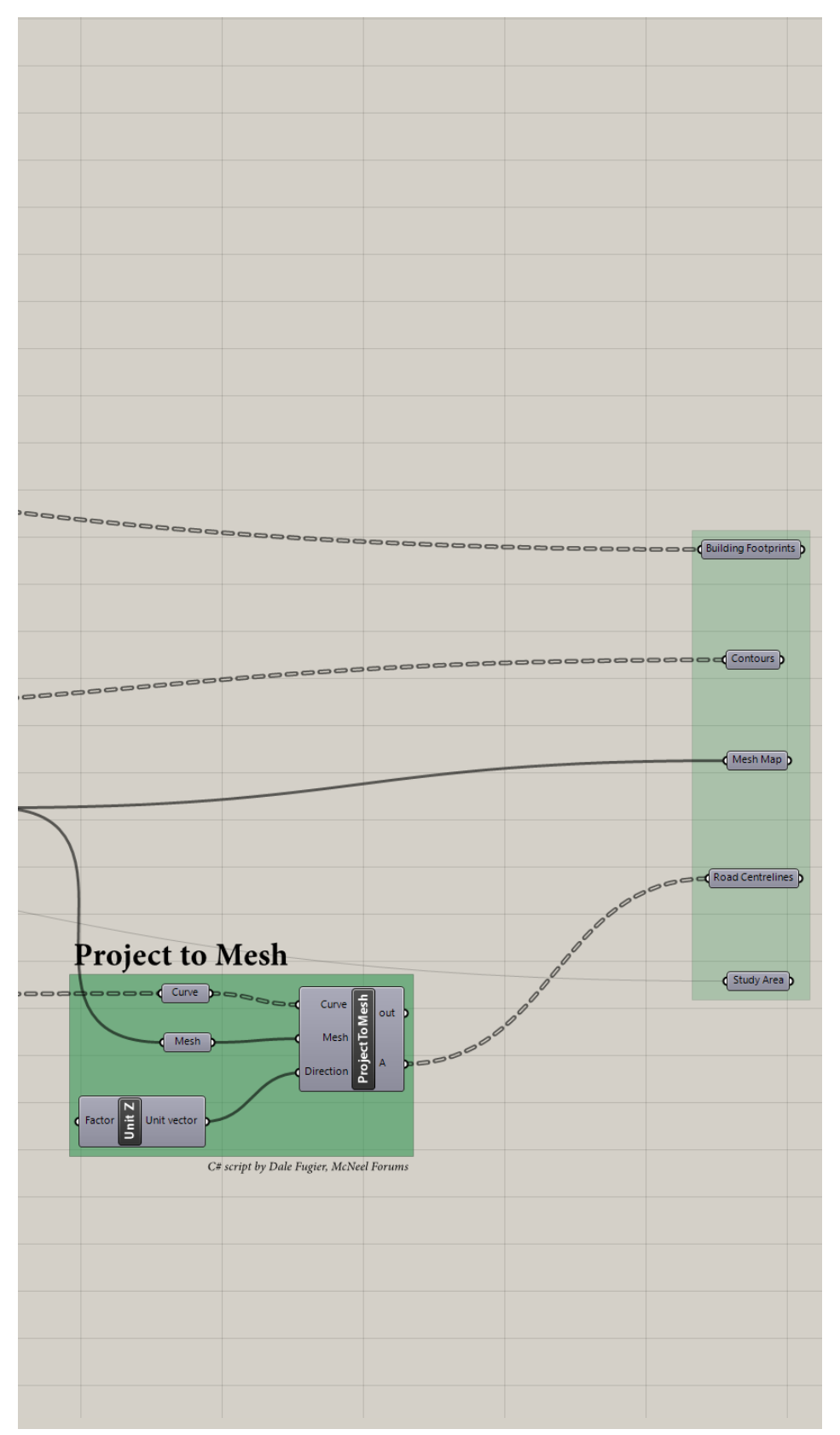

Head for the Hills: Computation Urban Design Prototyping for Complex Terrain

Part Two: Initial Graphical Evaluation

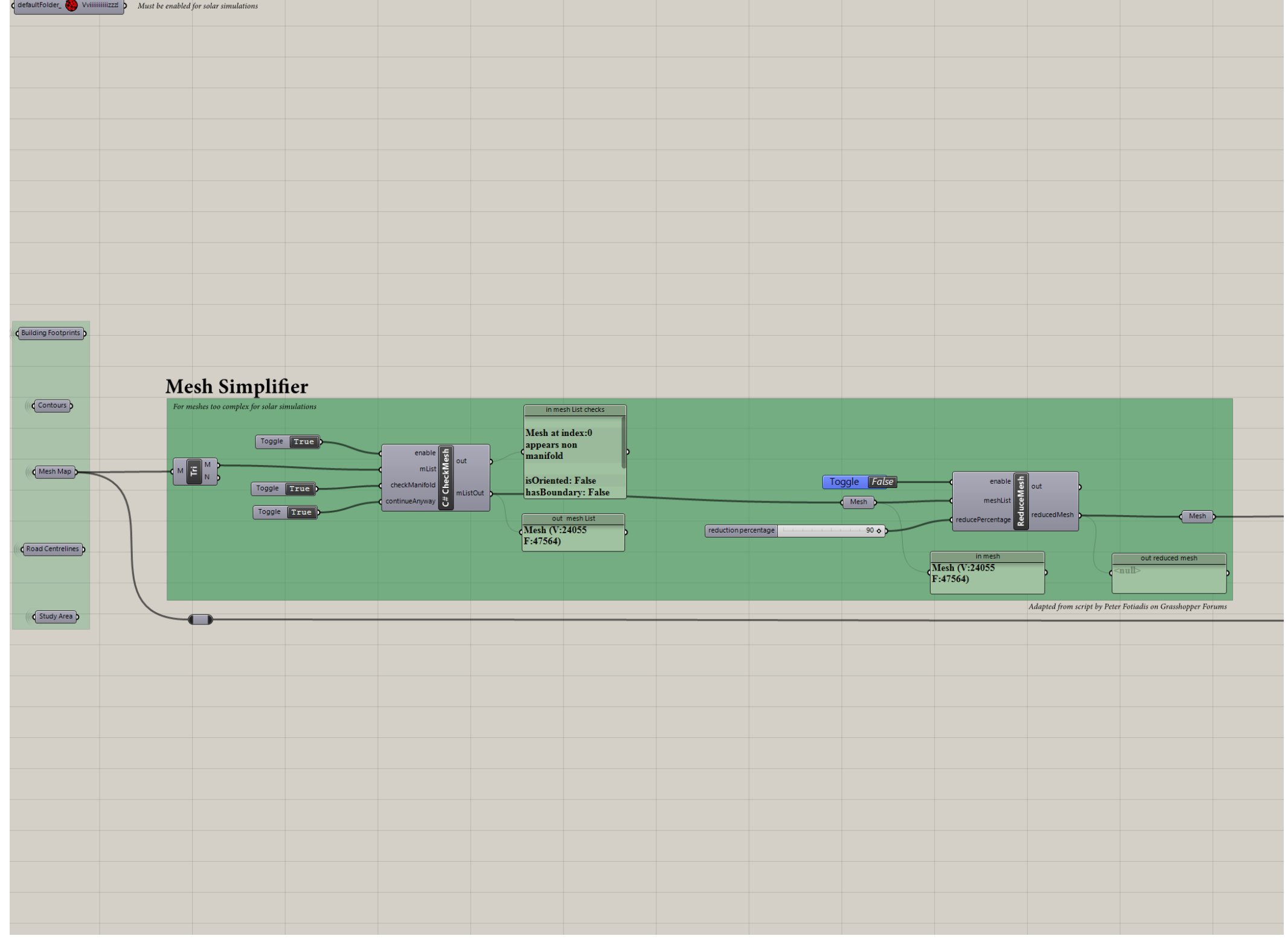




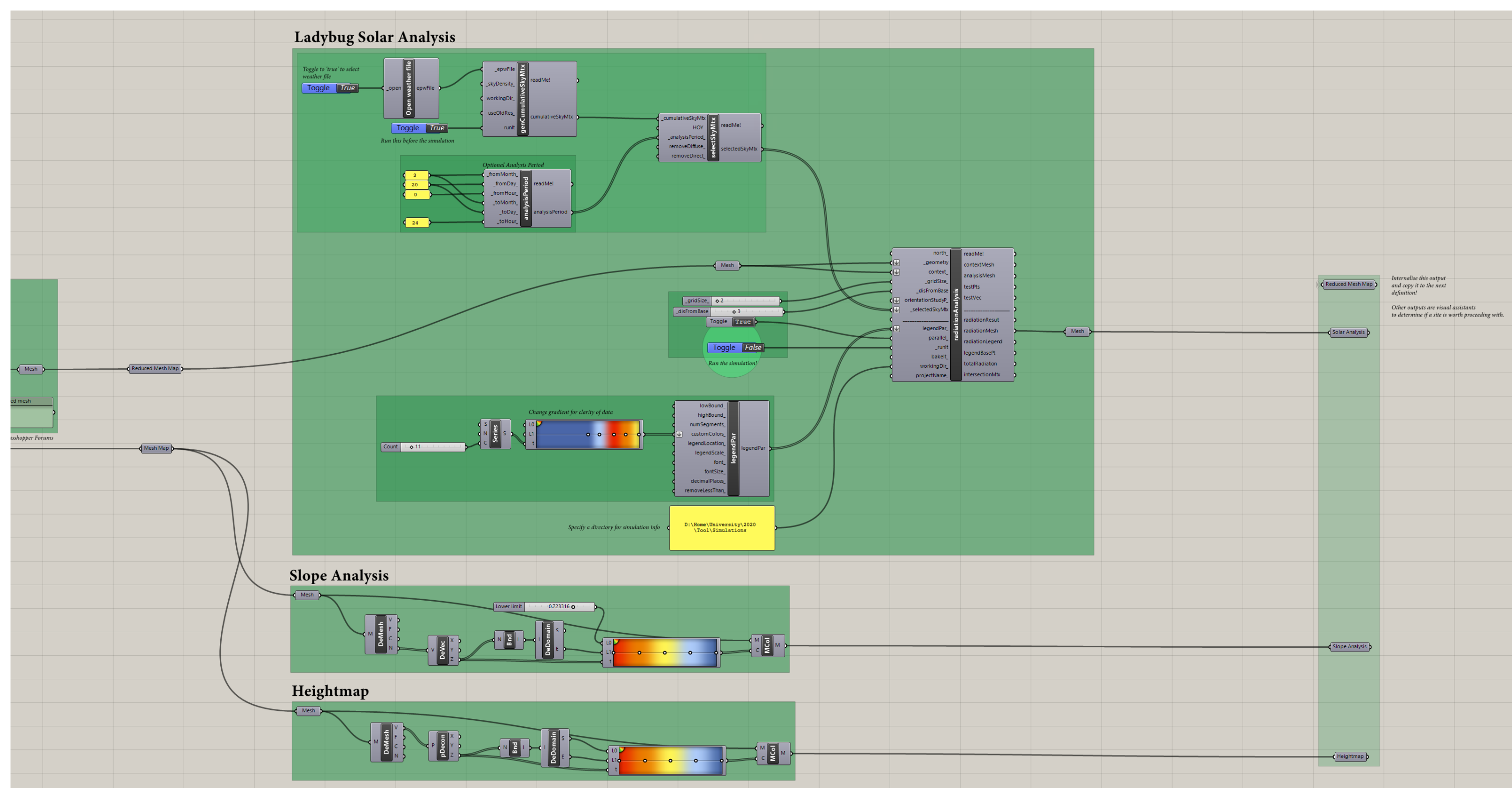


Head for the Hills: Computation Urban Design Prototyping for Complex Terrain Part Three: Blockmaker

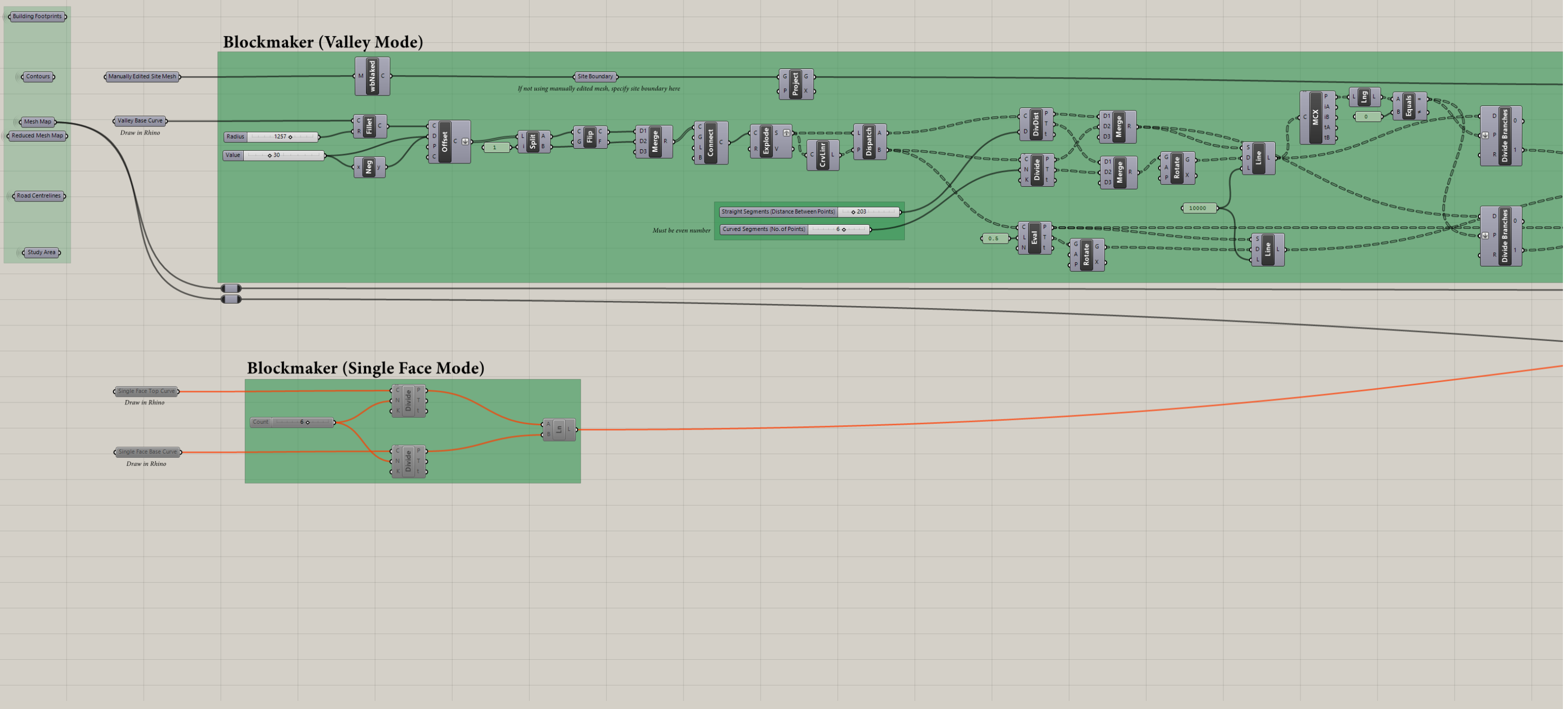




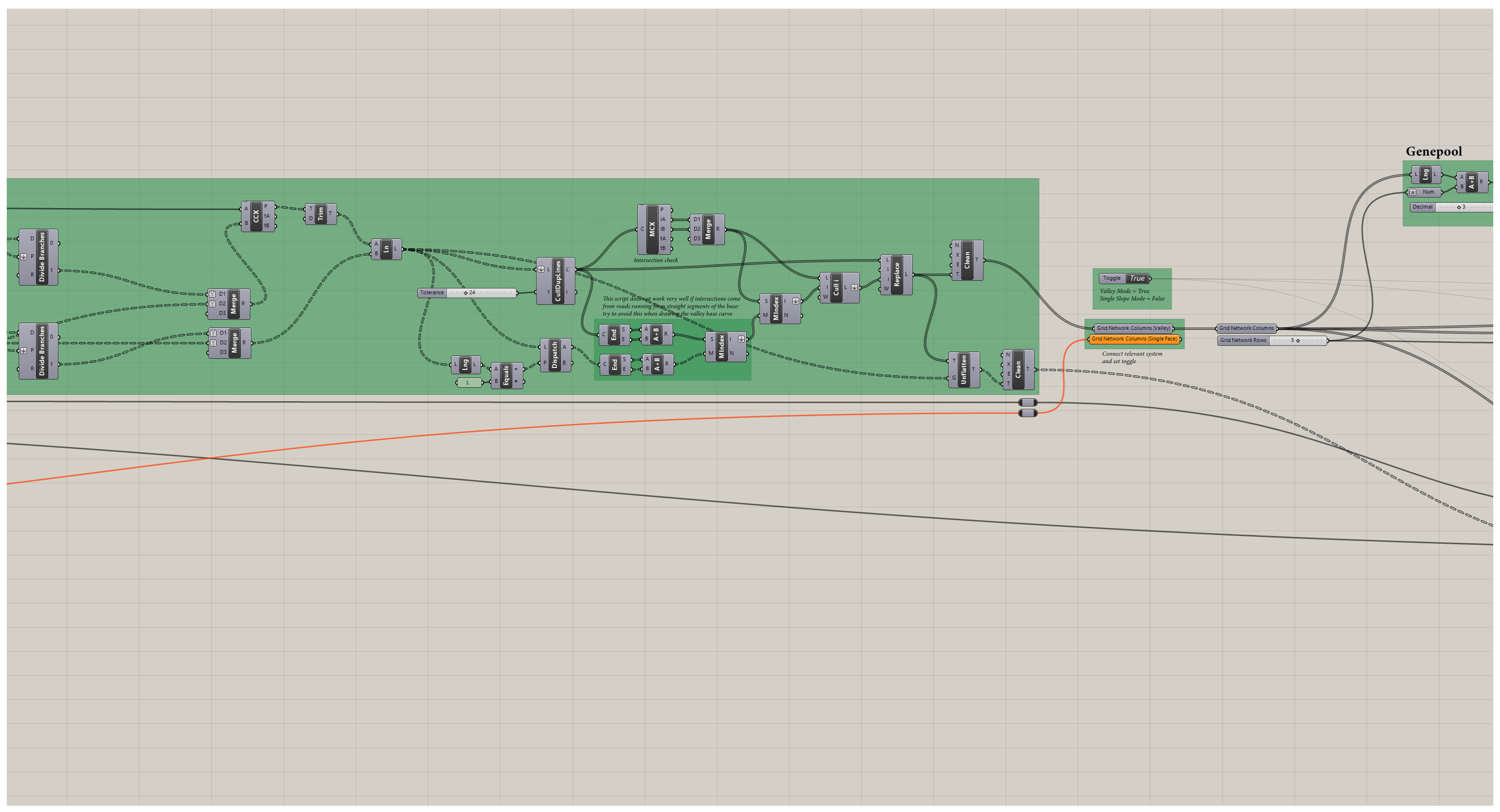




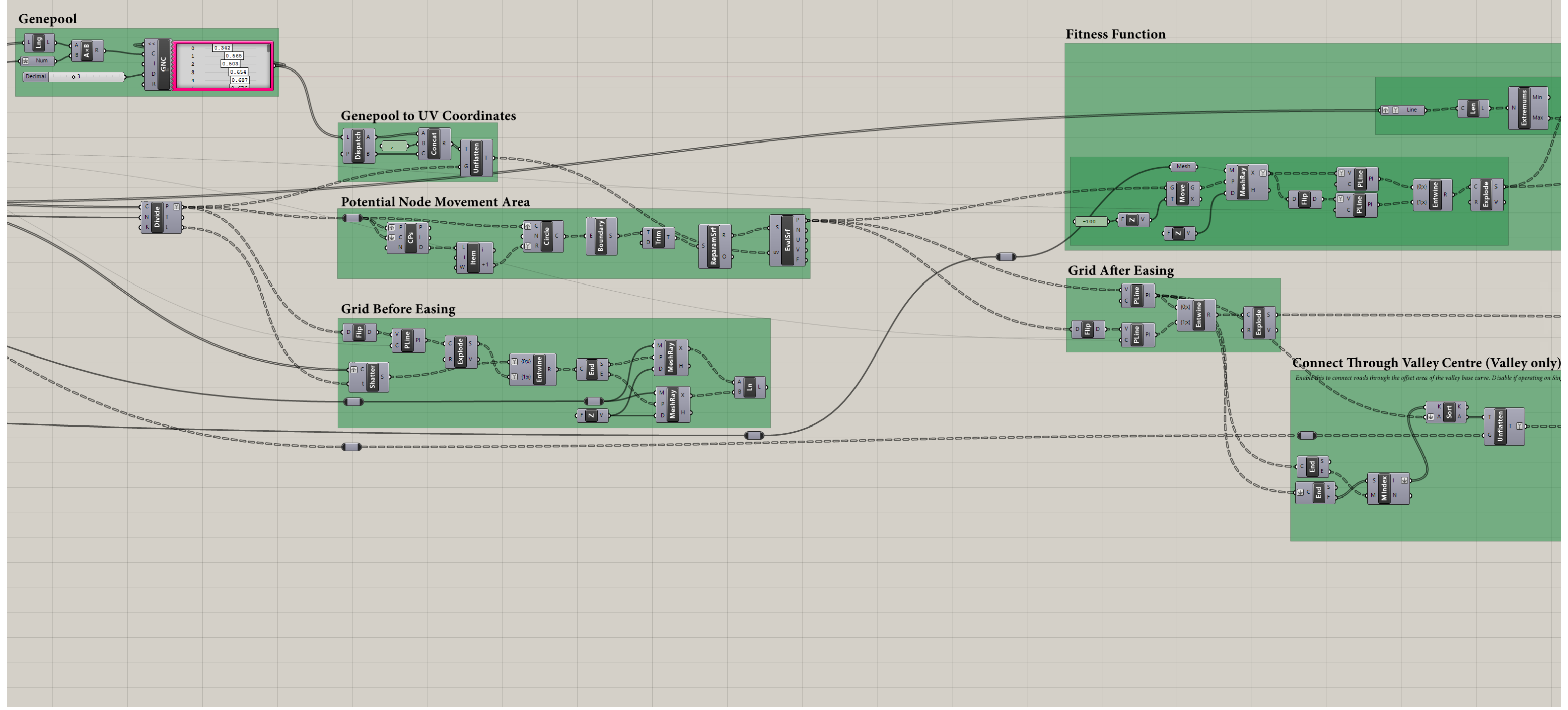




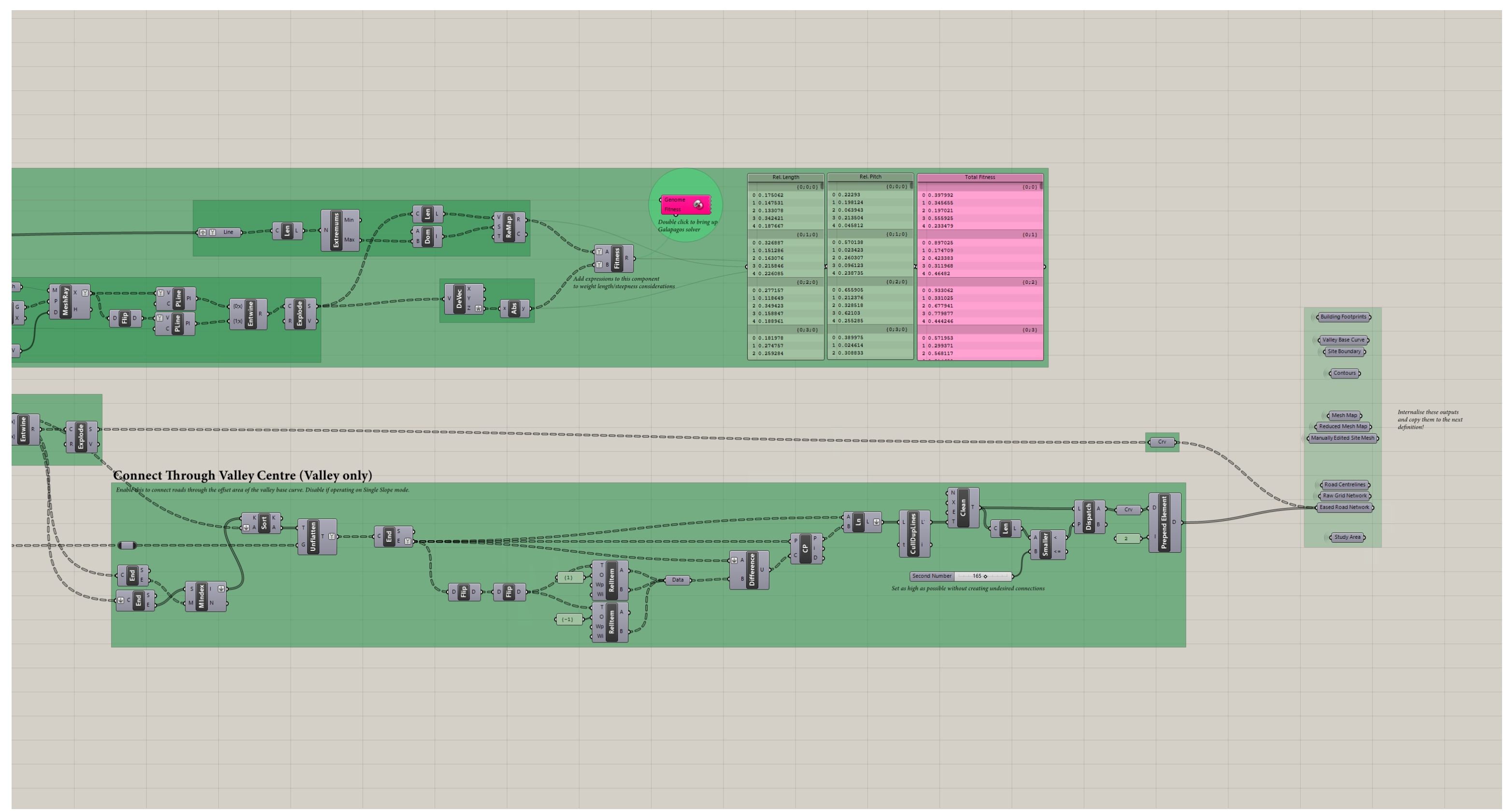


Head for the Hills: Computation Urban Design Prototyping for Complex Terrain

Aart Four: Roadmaker

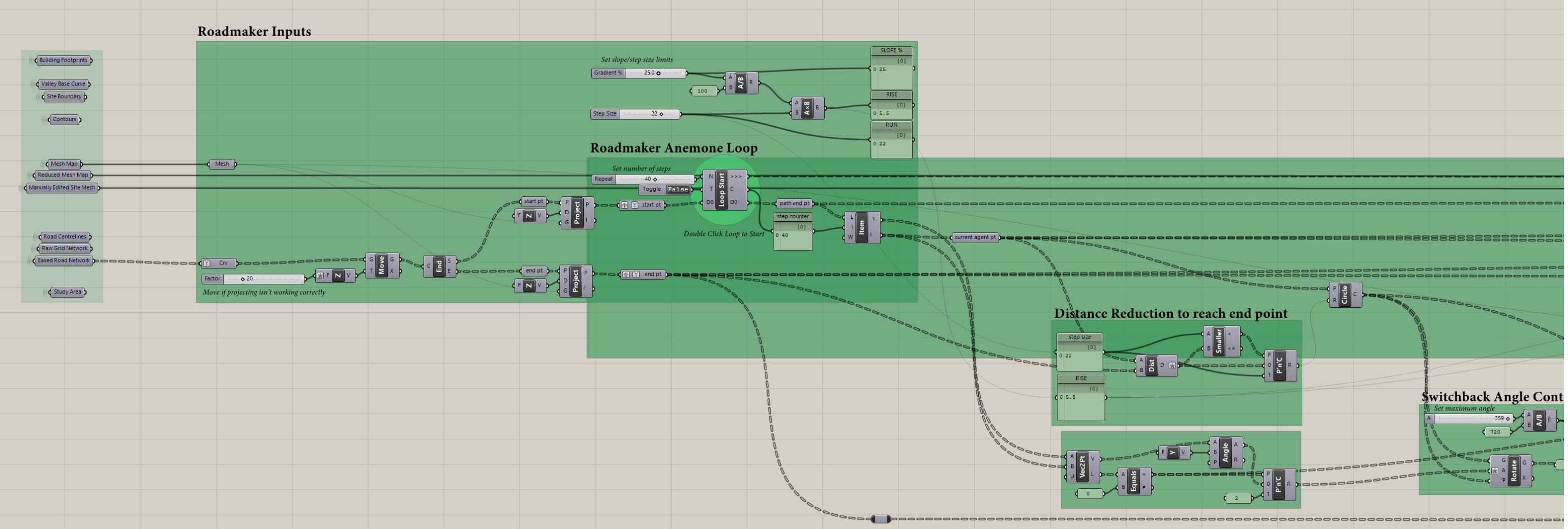




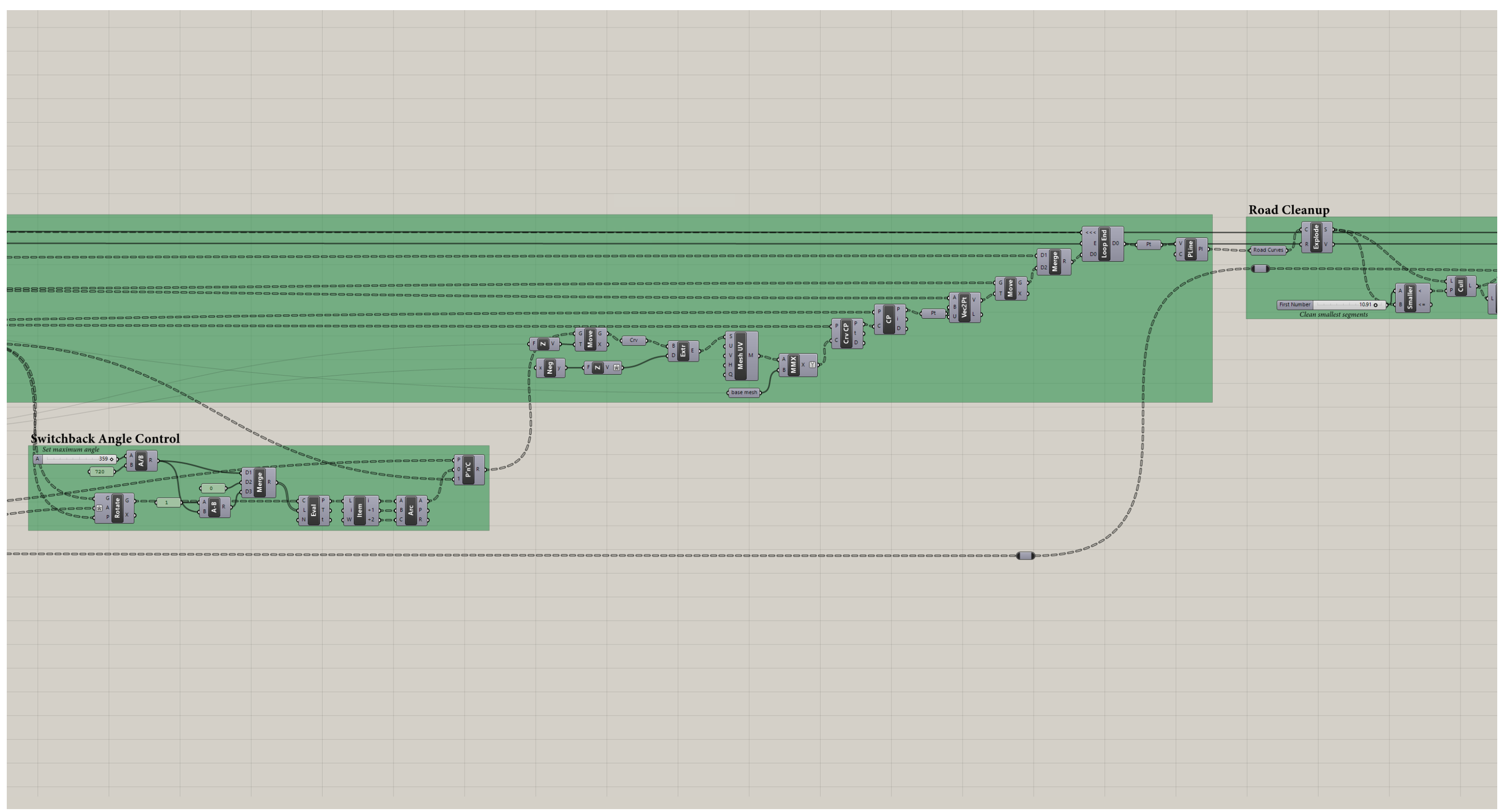




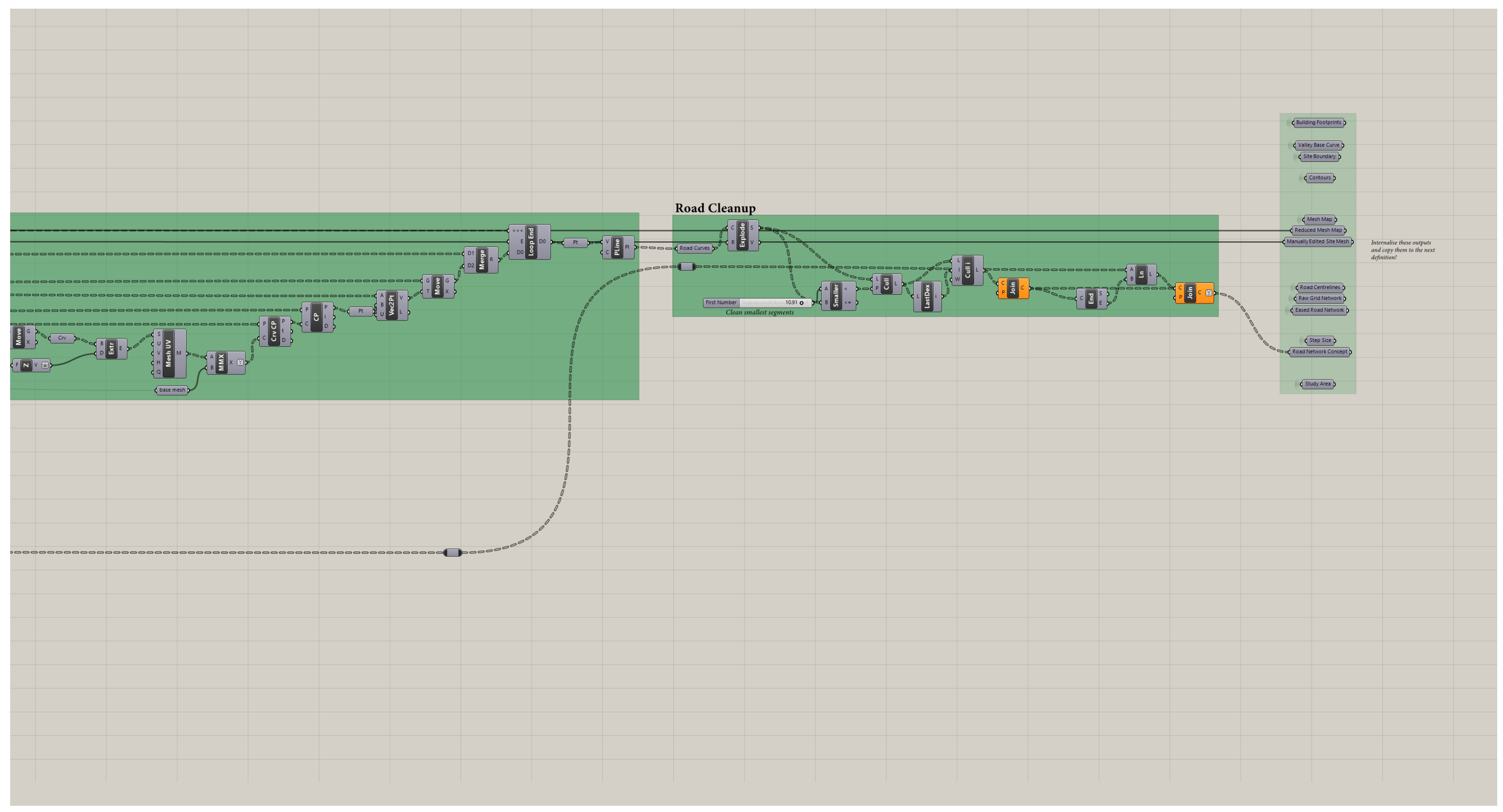


Head for the Hills: Computation Urban Design Prototyping for Complex Terrain

Part Five: Road Evaluation

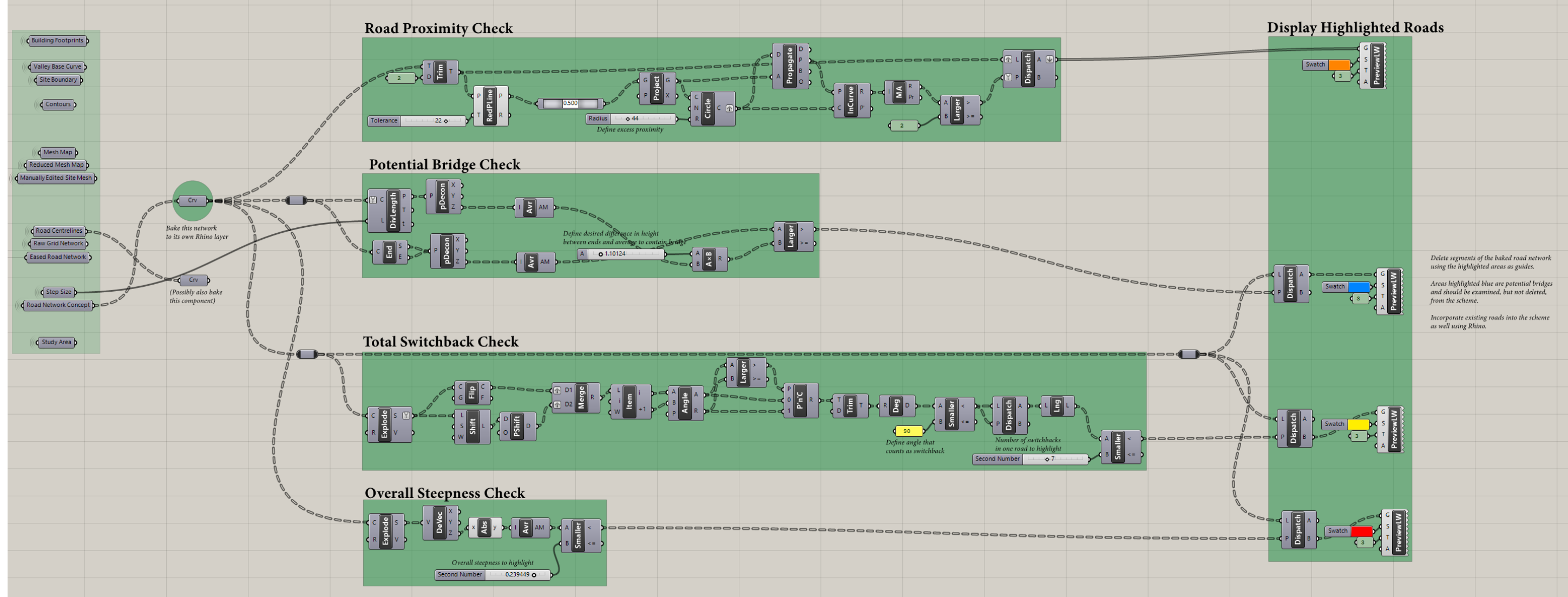




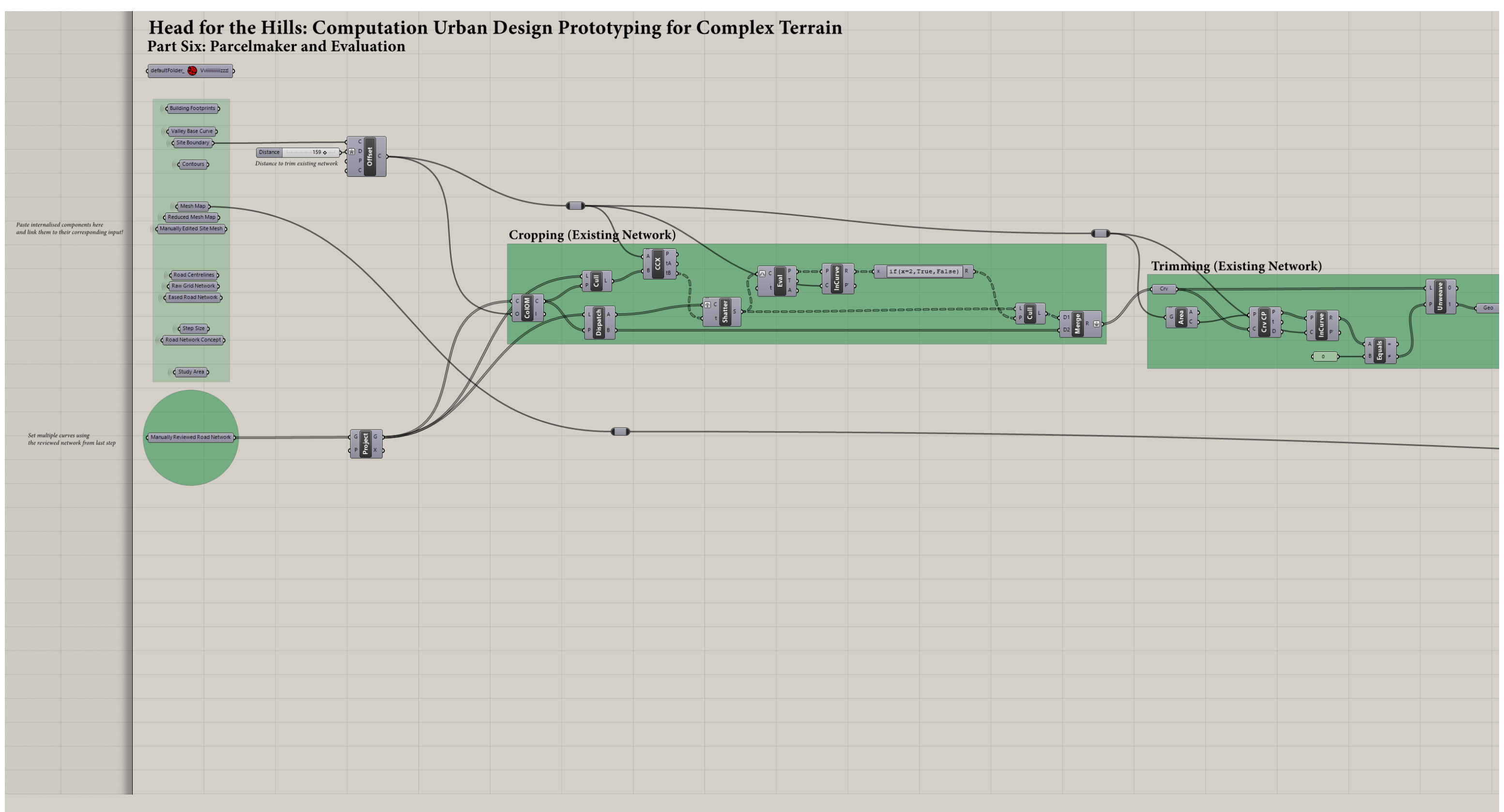




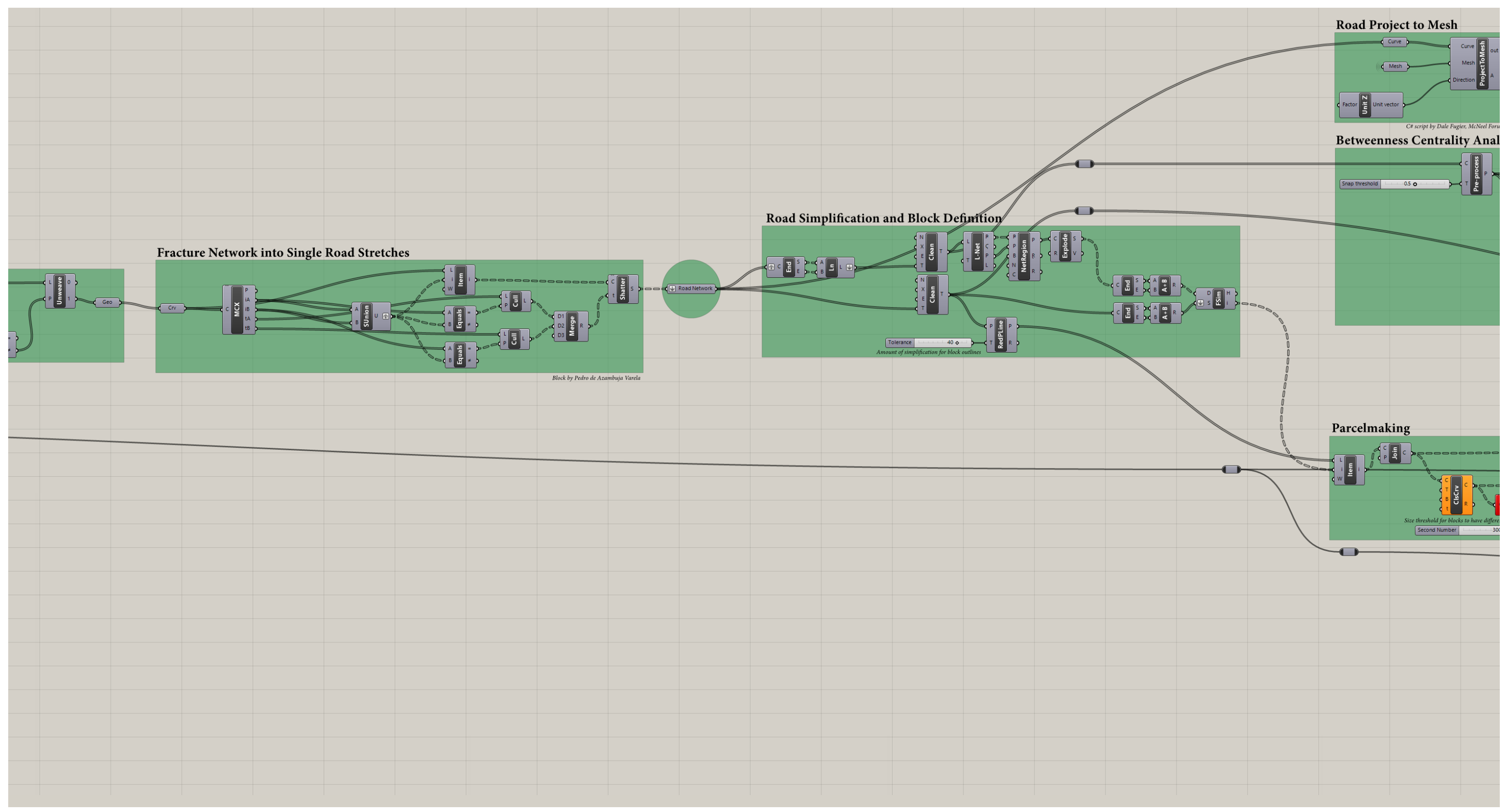




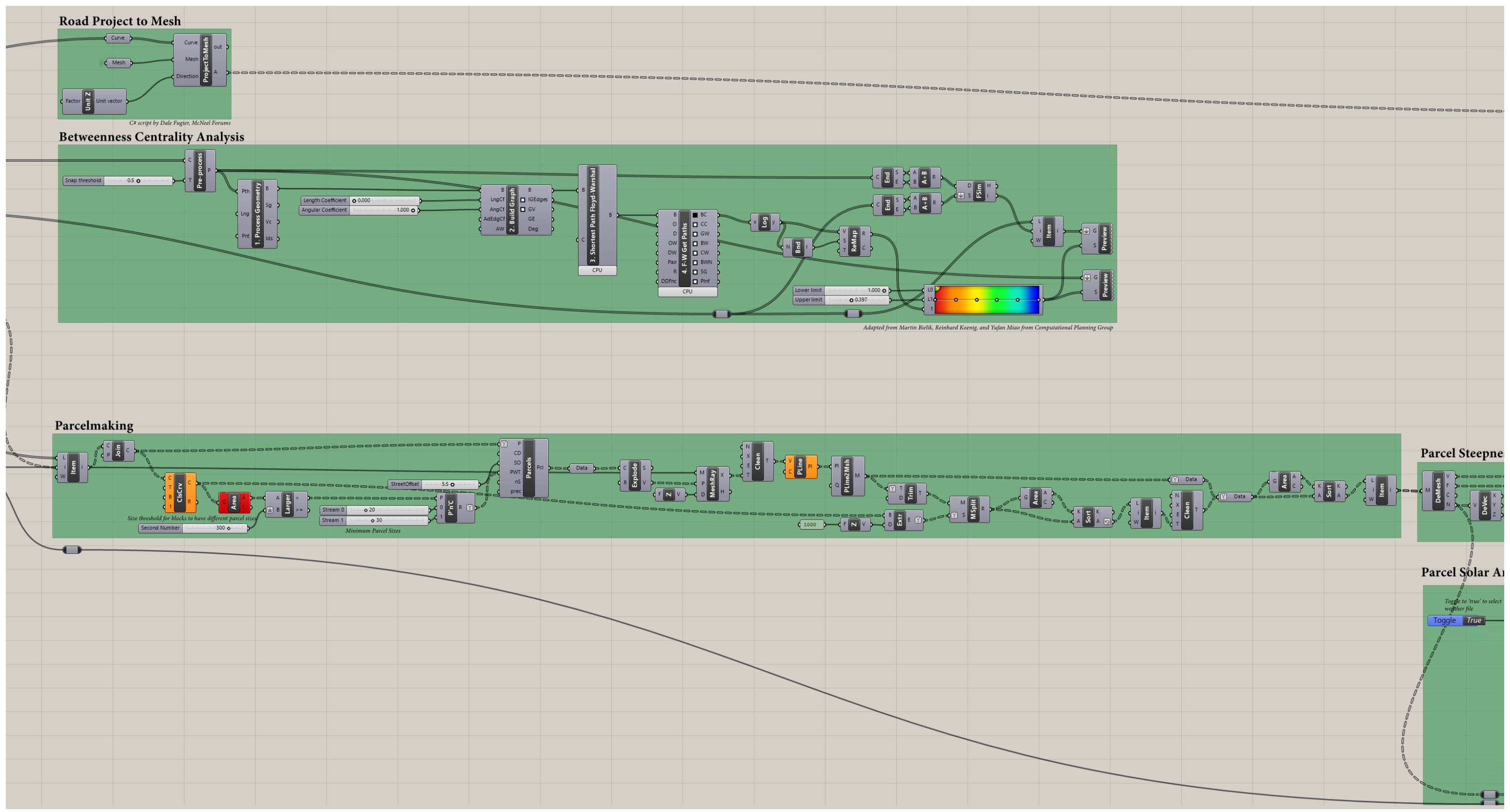




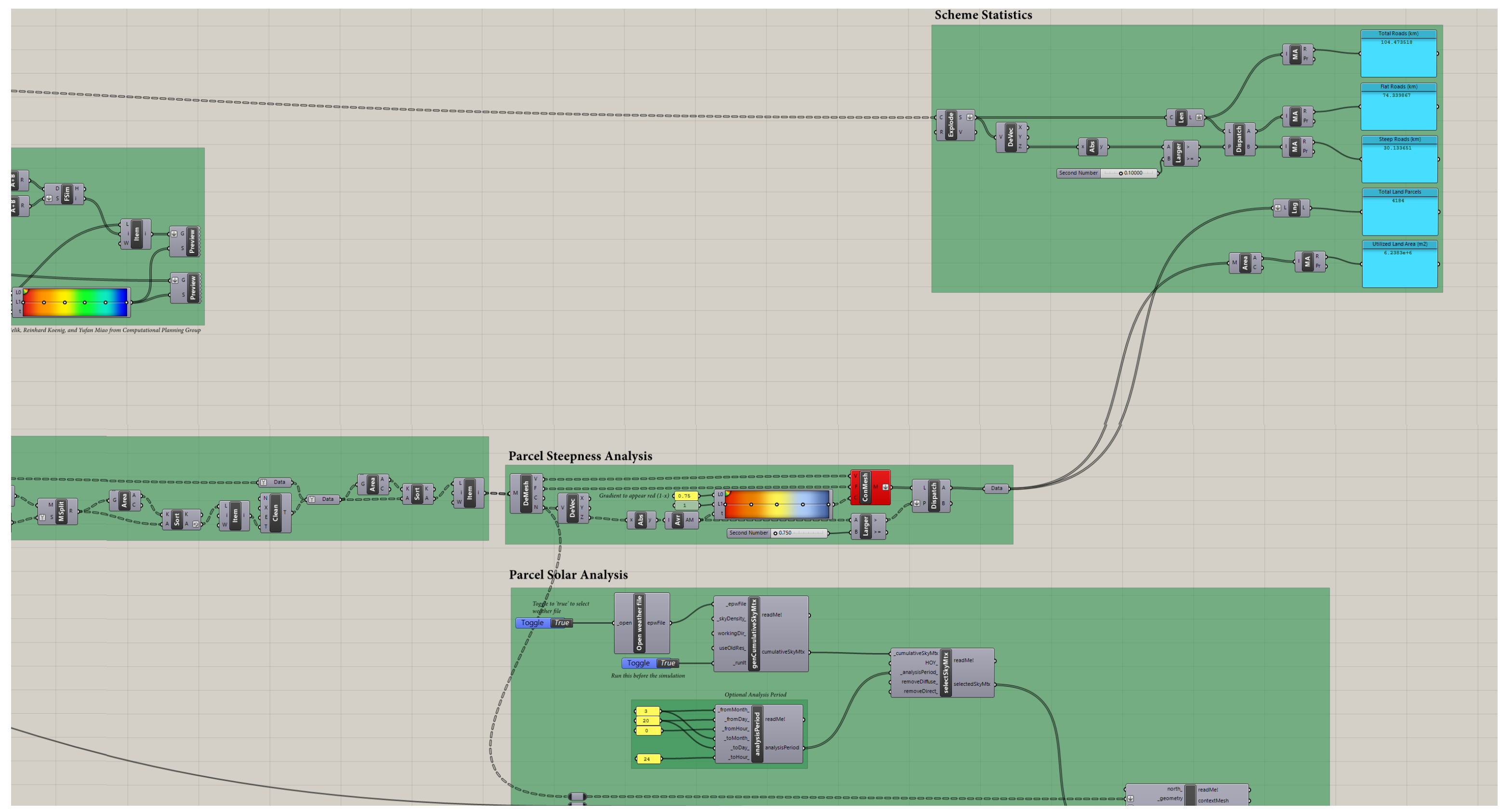




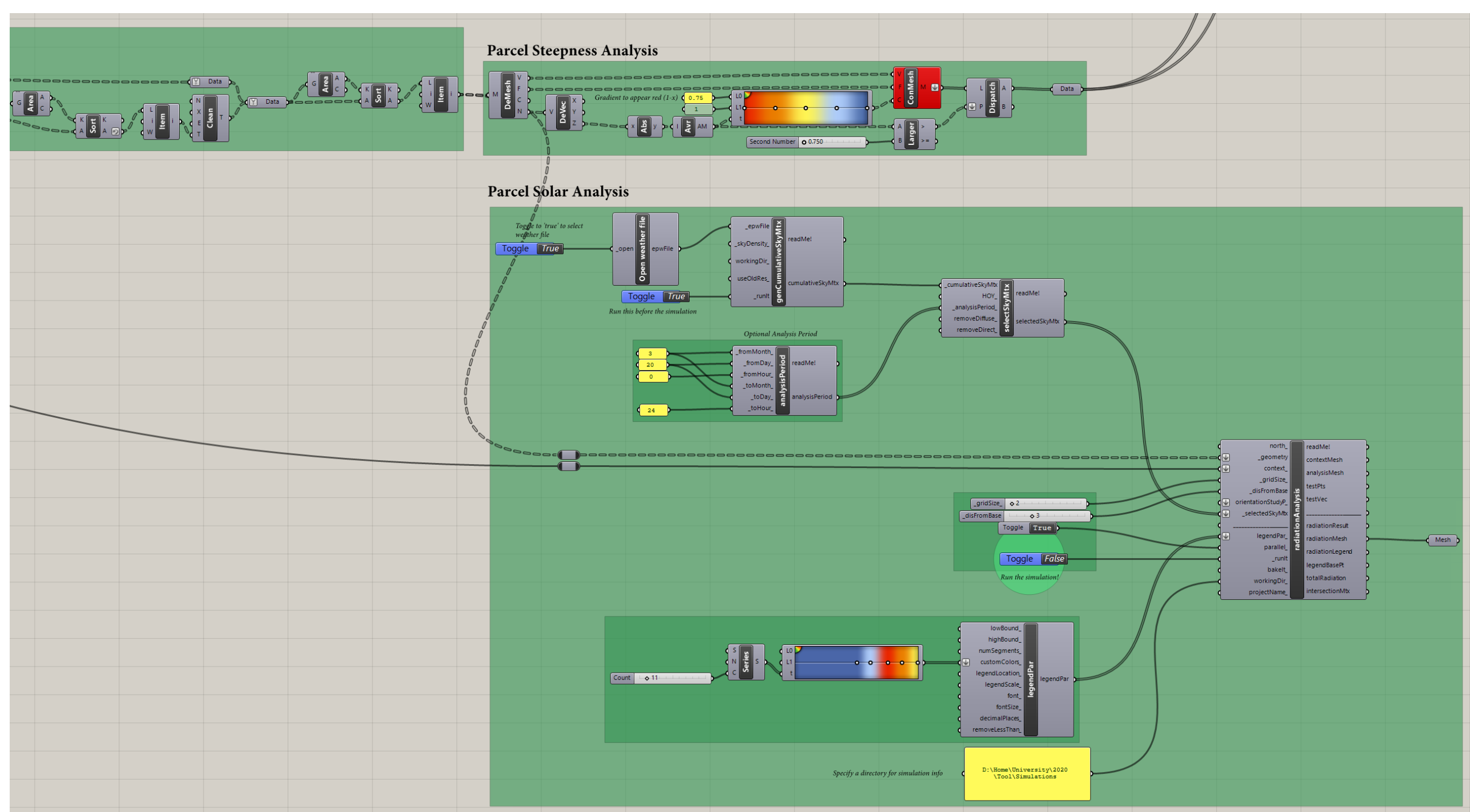


